PASJ: Publ. Astron. Soc. Japan , 1-??,

(C) 2018. Astronomical Society of Japan.

\title{
Subaru Weak Lensing Study of Seven Merging Clusters: Distributions of Mass and Baryons*
}

\author{
Nobuhiro OKABE \\ Astronomical Institute, Tohoku University, Aramaki, Aoba-ku, Sendai, 980-8578, Japan \\ okabe@astr.tohoku.ac.jp \\ Keiichi UMETSU \\ Institute of Astronomy and Astrophysics, Academia Sinica, \\ P.O. Box 23-141, Taipei 106, Taiwan, Republic of China \\ keiichi@asiaa.sinica.edu.tw
}

(Received 2007 February 24; accepted 2007 October 20)

\begin{abstract}
We present and compare projected distributions of mass, galaxies, and the intracluster medium (ICM) for a sample of merging clusters of galaxies based on the joint weak-lensing, optical photometric, and Xray analysis. Our sample comprises seven nearby Abell clusters, for which we have conducted systematic, deep imaging observations with Suprime-Cam on Subaru telescope. Our seven target clusters, representing various merging stages and conditions, allow us to investigate in details the physical interplay between dark matter, ICM, and galaxies associated with cluster formation and evolution. A1750 and A1758 are binary systems consisting of two cluster-sized components, A520, A754, A1758N, A1758S, and A1914 are on-going cluster mergers, and A2034 and A2142 are cold-front clusters. In the binary clusters, the projected mass, optical light, and X-ray distributions are overall similar and regular without significant substructures. On-going and cold-front merging clusters, on the other hand, reveal highly irregular mass distributions. Overall the mass distribution appears to be similar to the galaxy luminosity distribution, whereas their distributions are quite different from the ICM distribution in a various ways. We also measured for individual targets the global cluster parameters such as the cluster mass, the mass-to-light ratio, and the ICM temperature. A comparison of the ICM and virial temperatures of merging clusters from X-ray and weak-lensing analyses, respectively, shows that the ICM temperature of on-going and cold-front clusters is significantly higher than the cluster virial temperature by a factor of $\sim 2$. This temperature excess in the ICM could be explained by the effects of merger boosts.
\end{abstract}

Key words: gravitational lensing-X-rays: galaxies: clusters - galaxies: clusters: individual (A520, A754, A1750, A1758, A1914, A2034, A2142)

\section{INTRODUCTION}

In hierarchical structure formation models based on the Cold Dark Matter (CDM) scenario, small structures form first, and they merge to form larger, more massive objects. Since clusters of galaxies are the largest gravitationally bound objects in the universe, they are still forming through mergers of sub-clusters and smaller groups of galaxies along the filamentary structure within which they are embedded. The cluster merger is one of the most energetic events in the universe because of its highest binding energy and huge energy release. Dark matter, which is the dominant mass component of clusters, governs the dynamics of cluster mergers, and drives various phenomena in the hot, diffuse intracluster medium (ICM). Colliding substructures with high collision velocities trigger shocks or turbulences in the ICM. Merger shocks increase the temperature and entropy of the ICM, resulting in increasing the X-ray luminosity of the clus-

\footnotetext{
* Based on data collected at Subaru Telescope, which is operated by the National Astronomical Observatory of Japan.
}

ter. The merger-induced shocks and turbulences generate relativistic particles, which then produce synchrotron radio emission and inverse-Compton emission on the Cosmic Microwave Background. We however have not yet understood the cluster merger phenomena and the relationships between the ICM and dark matter in detail.

Observationally, cluster mergers have been investigated based on optical measures of cluster dynamics, namely line-of-sight velocity distributions of cluster member galaxies as well as optical appearance of substructures. Optical evidence of substructures were reported by many authors based on apparent concentrations of galaxies in projected space (e.g., Abell, Neyman, \& Scott 1964; Baier \& Ziener 1977; Geller \& Beers 1982), although a detection of subclustering of cluster galaxies is not direct evidence for the existence of underlying mass component. On the other hand, one can investigate the galaxy dynamics in clusters by examining distribution of galaxies both in spatial and velocity space (e.g. Zabludoff et al. 1993; Ashman, Bird \& Zepf 1994),

In recent years, X-ray satellites such as $A S C A, R O S A T$, Chandra, XMM-Newton and Suzaku make it possible to 
study the physical processes in the ICM involved in the formation and evolution of clusters. In particular, precise spectro-imaging data of Chandra and XMM-Newton allow us to derive the temperature, pressure, and entropy maps of the ICM, which are useful tools to study the cluster merger process. Recent Chandra and XMM-Newton observations have revealed a highly-complex X-ray morphology of merging clusters (e.g. Govoni et al. 2004; Henry, Finoguenov, \& Briel 2004; Finoguenov, Böhringer, \& Zhang 2005). Markevitch et al. (2000) discovered an unexpected ICM structure of contact discontinuities, which are called "cold fronts". However, although such X-ray observations of the ICM physics provide us with detailed information of the cluster merger, X-ray study alone is not sufficient for understanding of complex physical processes acting during the cluster merger. This is because cluster mergers are dynamically governed by dark matter which mainly accounts for $\sim 80 \%$ of the total mass of clusters. Further, the ICM in merging clusters is not in, or close to, hydrostatic equilibrium, so that the X-ray determined total mass of merging clusters, under the assumption of hydrostatic equilibrium, will be different from the gravitational mass of the clusters.

Gravitational lensing effects on background galaxies, on the other hand, are powerful, unique tools to study the mass distributions in clusters regardless of the physical/dynamical state of matter in the systems. Weak lensing image distortions of background galaxy images can be used to map the distribution of matter in clusters on scales of $\sim 100 \mathrm{kpc}$ to $1-2 \mathrm{Mpc}$ scales in a model-independent way (see, e.g., Bartelmann \& Schneider 2001; Umetsu, Tada, \& Futamase 1999). Therefore weak lensing enables the direct study of mass in clusters even when the clusters are in the process of pre/mid/post merging, where the assumption of hydrostatic equilibrium or isothermality are no more valid.

In this way, weak lensing, X-ray, and optical photometric observations provide complementary information of the physical properties of clusters. Hence, a joint analysis of weak lensing, X-ray, and optical photometric observations will yield a comprehensive picture of the physical state of ICM, dark matter and member galaxies.

In this paper we present weak-lensing mass distributions of a sample of seven nearby merging clusters, reconstructed using weak shear data taken with SuprimeCam on the $8.2 \mathrm{~m}$ Subaru telescope, and make a quantitative comparison of the mass maps with X-ray and optical galaxy distributions. The Subaru/Suprime-Cam is the most ideal, working instrument for weak lensing shape measurements of background galaxies because of its wide field-of-view of $34^{\prime} \times 27^{\prime}$, photon collecting power of the $8.2 \mathrm{~m}$ mirror, and excellent image quality with stable, small PSF anisotropy (e.g., Miyazaki et al. 2002; Hamana et al. 2003; Sato et al. 2003; Broadhurst et al. 2005). We selected for the Subaru weak lensing observations seven nearby merging clusters $(0.05 \lesssim z \lesssim 0.28)$ of different merging stages and properties, A520, A754, A1750, A1758, A1914, A2034 and A2142, for which archival Xray data of Chandra and/or XMM-Newton are available.
Detailed analysis results of individual clusters will be presented elsewhere. Details of our X-ray, optical and weaklensing analyses are presented in $\S 2$. In $\S 3$, we compare the resulting projected distributions of mass, optical luminosity density, and X-ray emission for our sample of merging clusters. In $\S 4$ we derive global cluster parameters such as the cluster mass, the cluster mass-to-light ratio, and the ICM temperature. Finally, in $\S 5$, we summarize the projected offset distributions of cluster galaxies and ICM with respect to the mass, and discusss the implications of our results for understanding of cluster merger phenomena.

Throughout the paper we adopt the concordance cosmology of $\Omega_{m 0}=0.3, \Omega_{\Lambda}=0.7$ and $H_{0}=70 \mathrm{kms}^{-1} \mathrm{Mpc}^{-1}$, and use the $\mathrm{AB}$ magnitude system.

\section{DATA ANALYSIS}

\subsection{Data Preparation}

\subsubsection{Subaru Data Analysis}

We observed all of the target clusters, except A520 for which archival data are available (SMOKA), with Subaru/Suprime-Cam (ID: S05A-159, PI: N. Okabe). For each target cluster we chose $R_{\mathrm{c}}$ or $i^{\prime}$ band filters for the weak lensing shape analysis, and $g^{\prime}$ or $V$ for separating member and background galaxies based on the colormagnitude diagram. All of our observations were taken using the AG (acquisition and guide) probe for guide stars, while the archival $i^{\prime}$-band data of A520 were taken with and without guide probe on 17th November 2001 and 19th October 2001, respectively. We retrieved from SMOKA a total of seven $i^{\prime}$-band images taken under good seeing conditions $\left(\sim 0 !^{\prime \prime} 6\right)$, and analyzed separately the following three imaging data sets: (A) all of seven $i^{\prime}$ images taken both with and without guide probe $(7 \times 240$ s exposure $)$, (B) four $i^{\prime}$ images taken without guide probe $(4 \times 240 \mathrm{~s}$ exposure), and (C) three $i^{\prime}$ images taken with guide probe $(3 \times 240$ s exposure $)$. Our main analysis results are based on the deepest data set (A) (see §3.7), which was also used by Mahdavi et al. (2007) for their multi-telescope, multi-bandpass weak lensing analysis of the cluster. We compare in Appendix the resulting mass maps derived from the thee different imaging data sets. We note that the A520 data were taken with large dithering offsets of $\approx 2$ ' 3 , whereas the other cluster data were taken with a dithering offset of $1^{\prime}$. A summary of observation parameters and conditions is given in Table 1 .

Each Subaru data set was reduced using the software SDFRED (Yagi et al. 2002; Ouchi et al. 2004). For each CCD frame we first estimated and subtracted the bias level by the median value in the overscan region. Next we carried out flat-fielding for all of the CCD frames to correct for the pixel-to-pixel variation in sensitivity. We then combined mosaic CCD images and corrected for the field distortion due to the camera optics. In order to obtain the local sky background map we first measure the median sky level in each mesh of a grid, and then compute a high-resolution background map via the $2 \mathrm{D}$ bilinear interpolation. Here we took the mesh size to be $32 \times 32$ pixels with 0 "' 202 pixel $^{-1}$. We subtract the sky background 
measured from our images. We matched the point spread function (PSF) between the frames and masked out areas and objects vignetted by the AG probe and bad pixels, such as satellite trails. Finally we generate a median combined image from these individual frames. Here stacking parameters such as the coordinate shifts, rotations, and scalings were determined by stellar objects common to all exposures. A summary of the seeing FWHMs of final reduced images for the weak lensing analysis is shown in Table 1.

In order to make an accurate comparison between optical and X-ray images, we performed astrometry using the IRAF 2.11 tasks (CCMAP and CCSETWCS) with our object catalogs generated using SExtractor (Bertin \& Arnouts 1996) and the USNO-A2.0 catalog as our reference. After the astrometry correction typical rms residuals are about 1 pixel $\left(=0 .{ }^{\prime \prime} 202\right)$.

\subsubsection{Chandra Data Analysis}

We used archival Chandra ACIS data for the X-ray analyses of A520, A754, A1914 and A2142. The X-ray observations are identified by their ID numbers (Obs. ID) in Table 1 . The data were reduced using the CIAO (version 3.3 for A520, A754 and A2142, and ver 3.2.2 for A1914) for the X-ray data processing. Standard screening was applied to the photon list. Bad pixels and columns were removed with ASCA grades 0, 2, 3, 4 and 6. The data were then cleaned of periods of anomalous background rates which are more than $3 \sigma$ significance from the mean value. We found an aspect offset in the reduced data of A754 (Obs.ID = 577), which was then corrected using the aspect calculator. We applied the CTI correction to our data with focal plane temperature of $-120^{\circ} \mathrm{C}$, because of the charge loss in ACIS-I CCD chips during transfer to the readout node. Background was derived from blank sky data. We normalized the background data so that the count rates in the $10-12 \mathrm{keV}$ band matched our observed count rates in the outer region of Chandra field-of-views.

The raw X-ray image was binned by a factor of 8 in each dimension (DETX and DETY). Point sources detected by WAVDETECT in the CIAO package was excluded from the images. X-ray images were adaptively smoothed, and background and exposure images were smoothed using the same kernel. Smoothed X-ray images were obtained by substituting the smoothed backgrounds and dividing them by smoothed exposure maps.

\subsubsection{XMM-Newton Data Analysis}

We used archival XMM-Newton data for A1750, A1758 and A2034 from three EPIC cameras (MOS1, MOS2 and $\mathrm{PN})$. X-ray observations are identified by their ID numbers (Obs. ID) in Table 1. We created calibrated event files using SAS version 6.5.0. The XMM-Newton data during high background flares were removed. To identify the high background periods, we generated light curves in the $>10 \mathrm{keV}$ energy band. The light curves were binned in $100 \mathrm{~s}$ intervals. We obtained good time intervals (GTIs) by applying $2 \sigma$ clipping. We confirmed that GTIs are consistent with previous works done by Belsole et al. (2004) and David \& Kempner (2004). The data were then filtered to leave only events with PATTERN $\leq 12$ and
\#XMMEA_EM for MOS and FLAG $=0$ and PATTERN $\leq$ 4 for PN.

We followed the double-subtraction method (Arnaud et al. 2002) for background modeling. Background files were produced by taking into account three different background components: particle-induced background, cosmic X-ray background (CXB) and residual soft photon contamination. The particle background was estimated from the filter wheel closed (FWC) data released from the EPIC Background Working Group. The particle background was re-normalized in the $0.3-10 \mathrm{keV}$ energy band by the ratio of the observation counts over the FWC particle counts outside the XMM field-of-view. For a CXB background estimation, we used blank-sky background data of the same instrument, filter and mode as those used in each observation. The blank-sky background counts were normalized to match the observed event counts in a source-free annulus at large radius. We obtained soft $\mathrm{X}$-ray excess images by matching in the low energy band the background count rates to our observed count rates in the outer region of the XMM field-of-view. Finally these normalized background images were combined to form the total X-ray background.

The raw X-ray image was then smoothed by a factor of 64 in each dimension (DETX and DETY). Finally we obtained for each target adaptively smoothed, backgroundsubtracted and exposure-corrected mosaic of the MOS1, MOS2 and PN images, in the same manner as done for the Chandra data.

\subsection{Cluster Galaxy Selection}

We performed object detection and aperture photometry using SExtractor (Bertin \& Arnauts 1996) in dualimage mode with deep $i^{\prime}$ or $R_{\mathrm{c}}$ as the detection image, yielding a common object catalog for the two filters, $\left(g^{\prime}, R_{\mathrm{c}}\right)$ or $\left(V, i^{\prime}\right)$. We extracted all objects with isophotal area larger than 10 pixels above $3 \sigma$ pixel $^{-1}$ of the local sky level. We used MAG_AUTO and MAG_APER of SExtractor output as object's total magnitude and aperture magnitude, respectively. For aperture photometry the aperture size is set to 10 pixels (2."02), except 20 pixels (4."04) for A754 at a very low redshift of $z=0.0542$. We define for each target cluster a sample of cluster member galaxies according to their color $\left(g^{\prime}-R_{\mathrm{c}}\right.$ or $\left.V-i^{\prime}\right)$ and magnitude $\left(R_{\mathrm{c}}\right.$ or $\left.i^{\prime}\right)$. Figure 1 shows the color magnitude diagram (CMD) for one of our target clusters, A2142, after the removal of stellar objects. The CMD in Figure 1 exhibits a tight color-magnitude (CM) sequence of earlytype cluster galaxies, since the two filters bracket the restframe $4000 \AA$ break. We quantify the CM sequence of galaxies with a linear relation in color-magnitude space. We set the maximum magnitude of the linear CM relation to $R_{\mathrm{c}}, i^{\prime}=22 \mathrm{ABmag}$. It can be seen that the luminous end of $\mathrm{CM}$ sequence extends to the high-luminosity regime of $R_{\mathrm{c}} \approx 16 \mathrm{ABmag}$. For individual galaxies, we convert from apparent to absolute magnitudes by using the k-correction for early-type galaxies. Here we assume that all of the member galaxies in each target cluster are located at a single redshift summarized in Table 2. 
We measure the optical luminosity of an individual cluster target by summing all the cluster galaxies within a given radius from the cluster center. Here we define the cluster center to be either at the brightest cluster galaxy (hereafter BCG). To do this we use a sample of bright CMsequence galaxies with magnitudes $R_{\mathrm{c}}, i^{\prime}<22 \mathrm{ABmag}$. We note that BCGs tend to deviate from the linear CM relation. Hence we visually checked if such brightest galaxies were properly included in our cluster member sample, and included them if they were missing. A field correction is applied to the measured luminosity density of cluster galaxies to account for contamination by field galaxies: We estimate the background luminosity density from an annular region outside of the target region, and then subtract this background contribution from the observed luminosity density of the cluster.

In order to measure the total luminosity of cluster galaxies, we also need to correct for incompleteness of the sample due to the magnitude limit. We assume that cluster galaxies follow a Schechter luminosity function (Schechter 1976) of the form:

$$
\phi(L)=\frac{d N}{d L}=\frac{\phi^{*}}{L^{*}}\left(\frac{L}{L^{*}}\right)^{-p} \exp \left(-L / L^{*}\right) .
$$

By integrating $\phi(L)$ down to the luminosity cutoff corresponding to the magnitude limit $L_{\min }$ of our cluster member sample, we obtain the following relation between the total luminosity $L_{\text {tot }}$ and the observed luminosity $L_{\mathrm{obs}}$ of the cluster:

$$
L_{\text {tot }}=L_{\mathrm{obs}} \frac{\Gamma(2-p)}{\Gamma\left(2-p, L_{\lim } / L^{*}\right)},
$$

where $\Gamma(a, x)$ is the upper incomplete gamma function. We adopt the following parameters for the cluster luminosity function: $p=1.03, M_{R_{c}}^{*}=-21.89+5 \log (h)$ in $R_{\mathrm{c}^{-}}$ band and $p=0.70, M_{i^{\prime}}^{*}=-22.31+5 \log \left(h_{70}\right)$ in $i^{\prime}$-band (Goto et al. 2002), where $M^{*}$ is the absolute magnitude corresponding to the characteristic luminosity $L^{*}$. The resulting luminosity correction factors, $\Gamma(2-p) / \Gamma(2-$ $\left.p, L_{\lim } / L^{*}\right)$, are of order of unity as shown in Table 3 .

\subsection{Weak Lensing}

In the weak lensing analysis we aim to reconstruct from image distortions of background galaxies the dimensionless surface mass density

$$
\kappa(\boldsymbol{\theta})=\Sigma_{\mathrm{cr}}^{-1} \Sigma(\boldsymbol{\theta})
$$

of clusters in units of the critical surface mass density for gravitational lensing,

$$
\Sigma_{\mathrm{cr}}=\frac{c^{2}}{4 \pi G} \frac{D_{s}}{D_{d} D_{d s}},
$$

where $D_{s}, D_{d}$ and $D_{d s}$ are the angular diameter distances from the observer to the sources, from the observer to the deflecting lens, and from the lens to the sources. In the following we closely follow the standard notation of Bartelmann \& Schneider (2001).

\subsubsection{Weak Lensing Distortion Analysis}

We use for our weak lensing analysis the IMCAT package developed by $\mathrm{N}$. Kaiser ${ }^{1}$, following the formalism developed by Kaiser, Squires, \& Broadhurst (1995). We have modified the method somewhat following the procedures described in Erben et al. (2001). We used the same analysis pipeline as in Broadhurst, Takada, Umetsu et al. (2005), Medezinski, Broadhurst, Umetsu et al. (2007), and Umetsu \& Broadhurst (2007). Note that our weak lensing analysis on A520 is based on the deepest data set in Table 2, and a comparison of the mass reconstructions between different imaging data sets is given in Appendix, demonstrating the effects of different observation conditions and strategies on the weak lensing analysis.

We measure the image ellipticity $e_{\alpha}=$ $\left\{Q_{11}-Q_{22}, Q_{12}\right\} /\left(Q_{11}+Q_{22}\right)$ from the weighted quadrupole moments of the surface brightness of individual galaxies,

$$
Q_{\alpha \beta}=\int d^{2} \theta W(\theta) \theta_{\alpha} \theta_{\beta} I(\boldsymbol{\theta}) \quad(\alpha, \beta=1,2)
$$

where $I(\boldsymbol{\theta})$ is the surface brightness distribution of an object, $W(\theta)$ is a Gaussian window function matched to the size of the object.

Firstly the PSF anisotropy needs to be corrected using the star images as references:

$$
e_{\alpha}^{\prime}=e_{\alpha}-P_{s m}^{\alpha \beta} q_{\beta}^{*}
$$

where $P_{s m}$ is the smear polarisability tensor being close to diagonal, and $q_{\alpha}^{*}=\left(P_{* s m}\right)_{\alpha \beta}^{-1} e_{*}^{\beta}$ is the stellar anisotropy kernel. We select bright, unsaturated foreground stars identified in a branch of the circularized half-light radius $\left(r_{h}\right)$ vs. magnitude $\left(i^{\prime}\right)$ diagram to measure $q_{\alpha}^{*}$. In Table 2 we summarize basic information and statistics of the stellar samples used for PSF corrections in our weak lensing analysis. To obtain a smooth map of $q_{\alpha}^{*}$ used in equation (6), we divided the co-added mosaic image, whose size is about $\sim 11 \mathrm{~K} \times 9 \mathrm{~K}$ pixels, into $5 \times 4$ chunks. The chunk length is determined based on the typical coherent scale of PSF anisotropy patterns. By this, PSF anisotropy in individual chunks can be well described by fairly low-order polynomials. The typical number of stars per chunk is 20-50, depending on the field (see $N^{*}$ of Table 2). We fitted the $q^{*}$ in each chunk independently with secondorder bi-polynomials, $q_{*}^{\alpha}(\boldsymbol{\theta})$, in conjunction with iterative $\sigma$-clipping rejection on each component of the residual: $\delta e_{\alpha}^{*}=e_{\alpha}^{*}-P_{* s m}^{\alpha \beta} q_{\beta}^{*}(\boldsymbol{\theta})$. As summarized in Table 4, uncorrected ellipticity components of stellar objects have on average a mean of $1-2 \%$ with a few $\%$ of rms, or variation of PSF across the data field. On the other hand, the residual $\delta e_{\alpha}^{*}$ after correction is reduced to $\left|\overline{\delta e}_{\alpha}^{*}\right| \lesssim 10^{-4}$. After the anisotropic PSF correction the rms value of stellar ellipticities, $\sigma\left(\delta e^{*}\right) \equiv \sqrt{\left\langle\left|\delta e^{*}\right|^{2}\right\rangle}$, is reduced from a few $\%$ to $(4-8) \times 10^{-3}$. We show in Figure 3 distributions of stellar ellipticity components before and after the PSF anisotropy correction for our target clusters. Figure 4 shows the distortion fields of stellar ellipticities before and after the

\footnotetext{
http://www.ifa.hawaii/kaiser/IMCAT
} 
PSF anisotropy correction. From the rest of the object catalog, we select objects with ${\overline{r_{h}}}^{*} \lesssim r_{h} \lesssim 15$ pixels as a weak lensing galaxy sample, where $\bar{r}_{h}^{*}$ is the median value of stellar half-light radii (see Table 4), corresponding to the half median width of circularized PSF over the data field. An apparent magnitude cut off is also made to remove from the weak lensing galaxy sample bright foreground/cluster galaxies and very faint galaxies with noisy shape measurements. Table 5 summarizes the magnitude range and the mean surface number density $n_{g}$ of background galaxies for our sample of target clusters. Without color selection the $n_{g}$ is ranging from $n_{g} \approx 37 \mathrm{arcmin}^{-2}$ (A520) to $n_{g} \approx 72 \mathrm{arcmin}^{-2}$ (A2142), depending on the depth, the seeing condition of the observations, and the degree of contamination of cluster/foreground galaxies.

Second, we need to correct the isotropic smearing effect on image ellipticities caused by seeing and the Gaussian window function used for the shape measurements. The reduced shear $g_{\alpha}=\gamma_{\alpha} /(1-\kappa)$ can be estimated from

$$
g_{\alpha}=\left(P_{g}^{-1}\right)_{\alpha \beta} e_{\beta}^{\prime}
$$

with the pre-seeing shear polarizability tensor $P_{\alpha \beta}^{g}$. In the weak lensing limit where $|\kappa|,\left|\gamma_{\alpha}\right| \ll 1$, the image ellipticity is linearly proportional to the gravitational shear $\gamma_{\alpha}: g_{\alpha} \approx$ $\gamma_{\alpha} \approx\left(P_{g}^{-1}\right)_{\alpha \beta} e_{\beta}^{\prime}$. We follow the procedure described in Erben et al. (2001) to measure $P_{g}$ for an individual galaxy (see also $\S 3.4$ of Hetterscheidt et al. 2007). We adopt the scalar correction scheme, namely,

$$
\left(P_{g}\right)_{\alpha \beta}=\frac{1}{2} \operatorname{tr}\left[P_{g}\right] \delta_{\alpha \beta} \equiv P_{g}^{\mathrm{s}} \delta_{\alpha \beta}
$$

(Hudson et al. 1998; Hoekstra et al. 1998; Erben et al. 2001; Hettersheidt et al. 2007). However, the $P_{g}^{\text {s }}$ measured for individual galaxies are still noisy especially for small and faint galaxies. We thus adopt a smoothing scheme in object parameter space proposed by Van Waerbeke et al. (2000; see also Erben et al. 2001; Hamana et al. 2003). In this scheme we first identify $N$ neighbors for each object in $r_{g}$-mag parameter space. We then calculate over the local ensemble the median value $\left\langle P_{g}^{\mathrm{s}}\right\rangle$ of $P_{g}^{\mathrm{s}}$ and the variance $\sigma_{g}^{2}$ of $g=g_{1}+i g_{2}$ using equation (7). The dispersion $\sigma_{g}$ is used as an rms error of the shear estimate for individual galaxies. We adopt $N=30$. For each cluster field we compute the mean variance $\bar{\sigma}_{g}^{2}$ over the background galaxy sample. In Table 5 we listed the mean rms $\bar{\sigma}_{g} \equiv \sqrt{\bar{\sigma}_{g}^{2}}$ per galaxy, which is of the order of $\bar{\sigma}_{g} \approx 0.4$. Finally we use the following estimator for the reduced shear:

$$
g_{\alpha}=e_{\alpha}^{\prime} /\left\langle P_{g}^{\mathrm{s}}\right\rangle \text {. }
$$

\subsubsection{Weak Lensing Mass Reconstruction}

Having obtained the shear estimates for a sample of background galaxies, we then pixelize the distortion data into a regular grid of pixels using a Gaussian $w_{g}(\theta) \propto$ $\exp \left[-\theta^{2} / \theta_{g}^{2}\right]$ with $\theta_{g}=\mathrm{FWHM} / \sqrt{4 \ln 2}$. Further we incorporate in the pixelization a statistical weight $u_{g}$ for an individual galaxy, so that the smoothed estimate of the reduced shear field at an angular position $\boldsymbol{\theta}$ is written as

$$
\bar{g}_{\alpha}(\boldsymbol{\theta})=\frac{\sum_{i} w_{g}\left(\boldsymbol{\theta}-\boldsymbol{\theta}_{i}\right) u_{g, i} g_{\alpha, i}}{\sum_{i} w_{g}\left(\boldsymbol{\theta}-\boldsymbol{\theta}_{i}\right) u_{g, i}}
$$

where $g_{\alpha, i}$ is the reduced shear estimate of the $i$ th galaxy at angular position $\boldsymbol{\theta}_{i}$, and $u_{g, i}$ is the statistical weight of $i$ th galaxy which is taken as the inverse variance, $u_{g, i}=1 /\left(\sigma_{g, i}^{2}+\alpha^{2}\right)$, with $\sigma_{g, i}$ being the rms error for the shear estimate of $i$ th galaxy (see $\S 2.3 .1$ ) and $\alpha^{2}$ being the softening constant variance (Hamana et al. 2003). We choose $\alpha=0.4$, which is a typical value of the mean $\mathrm{rms}$ $\bar{\sigma}_{g}$ over the background sample. The case with $\alpha=0$ corresponds to an inverse-variance weighting. On the other hand, the limit $\alpha \gg \sigma_{g, i}$ yields a uniform weighting. We have confirmed that our results are insensitive to the choice of $\alpha$ (i.e., inverse-variance or uniform weighting) under the adopted smoothing parameters.

The error variance for the smoothed shear $\bar{g}=\bar{g}_{1}+i \bar{g}_{2}$ (10) is then given as

$$
\sigma_{\bar{g}}^{2}(\boldsymbol{\theta})=\frac{\sum_{i} w_{g, i}^{2} u_{g, i}^{2} \sigma_{g, i}^{2}}{\left(\sum_{i} w_{g, i} u_{g, i}\right)^{2}}
$$

where $w_{g . i}=w_{g}\left(\boldsymbol{\theta}-\boldsymbol{\theta}_{i}\right)$ and we have used $\left\langle g_{\alpha, i} g_{\beta, j}\right\rangle=$ $(1 / 2) \sigma_{g, i}^{2} \delta_{\alpha \beta}^{\mathrm{K}} \delta_{i j}^{\mathrm{K}}$ with $\delta_{\alpha \beta}^{\mathrm{K}}$ and $\delta_{i j}^{\mathrm{K}}$ being the Kronecker's delta.

We then invert the pixelized reduced-shear field (10) to obtain the lensing convergence field. In the map-making we assume the linear shearing in the weak-lensing limit, that is, $g_{\alpha}=\gamma_{\alpha} /(1-\kappa) \approx \gamma_{\alpha}$. We adopt the two inversion methods, namely, the Kaiser \& Squires inversion method (Kaiser \& Squires 1993) and the noise-filtering inversion method outlined in Seitz \& Schneider (2001). The first method makes use of the 2D Green function in an infinite space, while the latter is based on the finite-field solution of the inversion problem for the reconstruction kernel. The finite-field method must be used for a nearby cluster where the data field is dominated by positive, biased density field with $\langle\kappa\rangle>0$. In the linear map-making process, the pixelized shear field is weighted by the inverse of the variance (11). Note that this weighting scheme corresponds to using only the diagonal part of the noise covariance matrix, $N\left(\boldsymbol{\theta}_{i}, \boldsymbol{\theta}_{j}\right)=\left\langle\overline{\Delta g}\left(\boldsymbol{\theta}_{i}\right) \overline{\Delta g}\left(\boldsymbol{\theta}_{j}\right)\right\rangle$, which is only an approximation of the actual inverse noise weighting in the presence of pixel-to-pixel correlation due to nonlocal Gaussian smoothing. In Table 5 we summarize the Gaussian FWHM used in the pixelization and the rms noise level in the reconstructed $\kappa$ field for our sample of target clusters.

The smoothing scale is chosen so as to optimize the weak lensing detection of target mass structures, depending both on the size of the structure and the strength of noise power $\left(\propto \bar{\sigma}_{g}^{2} / n_{g}\right)$. Under the adopted smoothing parameters, the typical rms reconstruction error in the $\kappa$ map is $~ 0.02$ except for A1914, in which a subarcminscale $(\mathrm{FWHM}=0.75)$, high resolution reconstruction was adopted to resolve substructures (see $\S 3.4$ ).

\subsubsection{Red Background Galaxy sample}

As demonstrated by Broadhurst et al. (2005) and Medezinski et al. (2007), it is crucial to make a secure selection of background galaxies in order to minimize the 
dilution of the lensing signal by cluster/foreground galaxies and to make an accurate determination of the cluster mass. To do this, we define a sample of red background galaxies whose colors are redder than the CM sequence of cluster member galaxies due to large $k$-corrections. These red background galaxies are largely composed of early to mid-type galaxies at moderate redshifts (Medezinski et al. 2007). Cluster member galaxies are not expected to extend to these colors in any significant numbers because the intrinsically reddest class of cluster galaxies, i.e. E/S0 galaxies, are defined by the CM sequence and lie blueward of chosen sample limit, so that even large photometric errors will not carry them into our red sample.

In Table 6 we summarize our selection criteria for the red background sample and the resulting mean surface density of the red galaxies. The larger the cluster redshift, the redder the CM sequence of cluster galaxies due to larger $k$-corrections. Hence, the number of red background galaxies, selected in this way, will decrease with cluster redshift. In particular, A1758 is at a moderately high redshift of $z=0.279$, and there are very few such red galaxies remained. We therefore relaxed the selection criteria of the red background sample for the case of A1758 (see Table 6).

2.3.4. Tangential Distortion and Cluster Mass Profile

For an individual cluster, we derive an azimuthallyaveraged shear profile as a function of projected radius from the fiducial cluster center, which is chosen as the optical center (see $\S 2.2$ ).

The tangential component $g_{+}$is used to obtain the azimuthally averaged distortion due to lensing, and computed from the distortion coefficients $\left(g_{1}, g_{2}\right)$ of each object:

$$
g_{+}=-\left(g_{1} \cos 2 \phi+g_{2} \sin 2 \phi\right),
$$

where $\phi$ is the position angle of an object with respect to the cluster center, and the uncertainty in the $g_{+}$measurement is $\sigma \equiv \sigma_{g} / \sqrt{2}$ in terms of the rms error $\sigma_{g}$ for the reduced shear measurement.

The estimation of $g_{+}$only has significance when evaluated statistically over a large number of background galaxies because of the intrinsic spread in shapes and orientations as well as the measurement errors in the shape measurement. In radial bins we calculate the weighted average of the $g_{+} \mathrm{s}$ and its weighted error:

$$
\begin{aligned}
\left\langle g_{+}\left(\theta_{n}\right)\right\rangle & =\frac{\sum_{i} u_{g, i} g_{+, i}}{\sum_{i} u_{g, i}}, \\
\sigma_{+}\left(\theta_{n}\right) & =\sqrt{\frac{\sum_{i} u_{g, i}^{2} \sigma_{i}^{2}}{\left(\sum_{i} u_{g, i}\right)^{2}}},
\end{aligned}
$$

where the index $i$ runs over all of the objects located within the $n$th annulus with a median radius of $\theta_{n}$, and $u_{g, i}=1 /\left(\sigma_{g, i}^{2}+\alpha^{2}\right)$ is the inverse variance weight softened with $\alpha=0.4$ ( see $\S 2.3 .2)$.

For a parameter-free estimation of the cluster mass profile we use the aperture-densitometry, or so-called the $\zeta_{c^{-}}$ statistic (Fahlman et al. 1994; Clowe et al. 2000) of the form:

$$
\begin{aligned}
\zeta_{c}\left(\theta ; \theta_{\text {inn }}, \theta_{\text {out }}\right) & =\bar{\kappa}(<\theta)-\bar{\kappa}\left(\theta_{\text {inn }}<\theta<\theta_{\text {out }}\right) \\
& =2 \int_{\theta}^{\theta_{\text {inn }}} d \ln \theta^{\prime}\left\langle\gamma_{+}(\theta)\right\rangle \\
& +\frac{2}{1-\theta_{\text {inn }}^{2} / \theta_{\text {out }}^{2}} \int_{\theta_{\text {inn }}}^{\theta_{\text {out }}} d \ln \theta^{\prime}\left\langle\gamma_{+}(\theta)\right\rangle
\end{aligned}
$$

where $\theta_{\text {inn }}$ and $\theta_{\text {out }}$ are the inner and outer radii of the annular background region in which the mean background contribution, $\bar{\kappa}\left(\theta_{\text {inn }}<\theta<\theta_{\text {out }}\right)$, is estimated; The $\left\langle\gamma_{+}\right\rangle$is an azimuthal average of the tangential component of the gravitational shear, which we take $\left\langle\gamma_{+}(\theta)\right\rangle \approx\left\langle g_{+}(\theta)\right\rangle$ in the weak lensing limit. Then the projected mass of the cluster inside the projected radius $\theta$ is given as

$$
M_{\zeta}(<\theta)=\pi \theta^{2} \Sigma_{\mathrm{cr}} \zeta_{c}\left(\theta ; \theta_{\text {inn }}, \theta_{\text {out }}\right) .
$$

$M_{\zeta}(<\theta)$ is regarded as a lower bound to the true enclosed mass $M(<\theta)$, because of the subtraction of the mean $\kappa$ within the outer region, $\theta_{\text {inn }}<\theta<\theta_{\text {out }}$. Errors on $\zeta_{c}$ are calculated by propagating the rms errors $\sigma_{+}\left(\theta_{n}\right)$ (equation [14]) for the tangential shear measurement.

The lensing properties such as $\kappa$ and $\gamma_{\alpha}$ depend on the source redshift and the background cosmology through the critical lensing surface mass density, $\Sigma_{\mathrm{cr}}$, defined in equation (4). For all of the cluster targets, we assume the mean redshift of the red background galaxies is $\left\langle z_{s}\right\rangle=1$ (e.g., Broadhurst et al. 2005). Since our target clusters are located at low redshifts $\left(z_{d} \lesssim 0.2\right.$ except $z_{d}=0.279$ for A1758) $\Sigma_{\text {cr }}$ depends weakly on the source redshift as shown in Figure 2, so that a precise knowledge of the redshift distribution of background galaxies is not crucial (see, e.g., Bartelmann \& Schneider 2001).

In order to quantify and constrain the cluster mass properties we fit the following two different mass models to $\zeta_{c}$-statistic measurements: singular isothermal sphere (SIS) and universal density profile proposed by Navarro, Frenk, \& White (1996, hereafter NFW). The details of the halo models are given in Appendix. We parametrize our halo models using the cluster virial properties, such as the cluster virial mass, $M_{\mathrm{vir}}$, the cluster virial epoch, $z_{\mathrm{vir}}$, and the cluster virial radius, $r_{\text {vir }}$ :

$$
M_{\mathrm{vir}}=\frac{4 \pi}{3} \bar{\rho}\left(z_{\mathrm{vir}}\right) \Delta_{\mathrm{vir}} r_{\mathrm{vir}}^{3}
$$

where $\Delta_{\text {vir }}$ is the overdensity with respect to the mean cosmic density $\bar{\rho}\left(z_{\text {vir }}\right)$ at the cluster virial redshift, predicted by the dissipationless spherical tophat collapse model (Peeebles 1980; Eke, Cole, \& Frenk 1996; Bullock et al. 2001). We assume the cluster redshift $z_{d}$ is equal to the cluster virial redshift $z_{\text {vir }}$.

The SIS model has a one-parameter functional form described by the one-dimensional velocity dispersion $\sigma_{v}$ of the cluster, which is related with the cluster virial redshift $z_{\text {vir }}$ and the cluster virial mass $M_{\text {vir }}$ as

$$
\sigma_{v}\left(M_{\mathrm{vir}}, z_{\mathrm{vir}}\right)=\frac{1}{2} r_{\mathrm{vir}} H_{0} \sqrt{\Omega_{m 0} \Delta_{\mathrm{vir}}\left(1+z_{\mathrm{vir}}\right)^{3}}
$$

Thus, $\sigma_{v} \propto M_{\mathrm{vir}}^{1 / 3}$. Alternatively we can introduce the virial temperature of an SIS halo: 


$$
\mu m_{p} \sigma_{v}^{2}=k_{B} T_{\mathrm{SIS}}
$$

where $\mu=0.62$ is the mean molecular weight and $m_{p}$ is the proton mass.

On the other hand, the NFW model has a twoparameter functional form, and we take the virial mass, $M_{\mathrm{vir}}$, and the concentration parameter, $c_{\mathrm{vir}}=r_{\mathrm{vir}} / r_{s}$, with $r_{s}$ being the inner characteristic radius of the NFW profile.

\section{DISTRIBUTIONS OF MASS AND BARYONS IN MERGING CLUSTERS}

Here we present and compare in Figures 5-11 the resulting two dimensional maps of the convergence $\kappa$, optical luminosity density, and X-ray emission for our sample of merging clusters. Table 5 lists the properties of our background galaxy samples and the parameters relevant for weak lensing mass reconstructions. The cluster targets are selected to be on the various merging stages based on previous detailed X-ray studies, for which the physical relationship between mass and baryons though the merging processes is yet unknown. X-ray properties of the target clusters are summarized in Table 2. A1750 and A1758 are binary clusters which are presumably in a pre-merger phase. A2034 and A2142 are cold front clusters. A754, A1914 and A520 are on-going mergers, which have irregular temperature distributions and radio halos (Govoni et al. 2004).

\section{1. $A 754$}

A754 is an on-going merger at a redshift of $z=0.054$. For such a low redshift cluster $(z<0.1)$, the expected lensing signal is very low (Figure 2) and a weak lensing analysis is challenging. As seen from the Chandra X-ray contours in the top-right panel of Figure 5, there are two major gas components in the east and west sides of the data field. The west gas clump (XC in Figure 5) corresponds to the main cluster having a BCG. From this configuration, the east X-ray gas clump (XE in Figure 5) seems to have been running through the cluster center from the west. The XMM-Newton image with a wider field-of-view has shown a moving feature of the east clump consistent with the Chandra X-ray observation (Henry, Finoguenov, \& Briel. 2004). The X-ray contours of the east gas clump are compressed and elongated towards the north-east direction, indicating that the east gas clump is currently moving towards the northeast. Its averaged temperature within 9 arcmin radius is $10.0 \pm 0.3 \mathrm{keV}$ at the $90 \%$ confidence level (Markevitch et al. 2003; see also Table 2). The X-ray temperature maps of A754 were derived by Henry \& Briel (1995) with ROSAT data, Henriksen \& Markevitch (1996) with ASCA data, Markevitch et al. (2003) with Chandra data, and Henry, Finoguenov \& Briel (2004) with XMM-Newton data. Henry \& Markevitch (1996) obtained an X-ray temperature of $8.5-9.0 \mathrm{keV}$ from the ASCA data. Henry et al. (2004) derived an X-ray temperature within $12^{\prime}$ to be $8.6 \pm 0.1 \pm 0.6$ where the first and the second errors are statistical and systematic un- certainties at $1 \sigma$ confidence, respectively. The ASCA and XMM-Newton temperatures of A754 are somewhat lower than the Chandra temperature. However, the ASCA and Chandra measurements covered larger volumes of this ongoing merger. The Chandra temperature map shows a strongly irregular feature, where the cool gas is offset from the X-ray brightness peak towards the northeast by $\sim 200$ kpc.

Since this nearby cluster extends outside the fieldof-view of Subaru/Suprime-Cam, we used a finite-field method for reconstructing the mass distribution of A754 as shown in Figure 5. We also compared the $\kappa$ map derived using the Kaiser \& Squires method with the finitefield based $\kappa$ map. The $\kappa$ maps derived with the different methods are qualitatively similar, and the main features and quantitative properties of the mass peaks are consistent with each other within $1 \sigma$ reconstruction error, which means that the mass reconstruction is insensitive to the boundary conditions. The Gaussian FWHM used for the mass reconstruction is $1 ! 67$. The reconstructed $\kappa$ map shows two mass clumps in the west and east sides of the data field. The west clump is located in the X-ray cluster central region (Henry, Finoguenov \& Briel 2004), and hence we refer to this as the central mass clump ( $\mathrm{C}$ in Figure 5). We found moderate signal-to-noise ratios of 5.8 and 5.1 in the $\kappa$ map for the central and east mass (E in Figure 5) clumps, respectively, thanks to the superb image quality of Subaru/Suprime-Cam.

The peak locations in the luminosity map of cluster sequence galaxies are in good agreement with these mass substructures (bottom-left panel). The central mass peak coincides with the BCG (top-left panel). The east mass concentration contains a luminous, large elliptical galaxy.

The east mass clump is offset from the east X-ray clump towards the southwest, which is opposite to the moving direction of this gas clump (bottom-right panel). This kinematic feature is different from the case of the bullet cluster, 1E0657-56 (Clowe,Gonzale \& Markevitch 2004). The cool gas region (see Markevitch et al. 2003) is located outside the region enclosed by the $1 \sigma$ mass contour around the east mass clump.

A possible scenario explaining the observed merger geometry of the east mass and X-ray clumps, which cannot be simply explained by the ram-pressure stripping, is the following: the east mass substructure just reaches its apocenter of the merger orbit and falls back towards the center for its second impact. In the rest frame of the mass clump, the X-ray core, which is initially bounded in the mass clump, feels the force in the opposite direction to the acceleration (and moving) direction of the mass clump. This explains the observed configuration of the mass and gas clumps associated with the east substructure. As a result, the gas clump escapes away from the potential well of the mass substructure and would cool adiabatically as it expands.

\section{2. $A 1750$}

A1750 is a binary cluster at $z=0.086$, well studied by Einstein, ROSAT, ASCA and XMM-Newton X-ray satel- 
lites (Forman et al. 1981; Novicki, Jones, \& Donnelly 1998; Donnelly et al. 2001; Belsole et al. 2004). The XMM-Newton observation (Belsole et al. 2004) has shown that the southern cluster, A1750C, has a higher X-ray luminosity than the northern cluster, A1750N. The projected distance between the two X-ray peaks is about 900 kpc. The X-ray temperatures of A1750C and A1750N are $3.87 \pm 0.10 \mathrm{keV}$ and $2.84 \pm 0.12 \mathrm{keV}$ at $90 \%$ confidence, respectively, (Belsole et al. 2004). The projected separation is therefore shorter than the sum the two virial radii predicted by the $M-T_{X}$ relation. Hence, it is likely that the two clusters just started to interact with each other. Indeed, the temperature in the middle region of the two clusters is higher than the cluster temperatures: $T_{X}=5.12_{-0.69}^{+0.77} \mathrm{keV}$. Belsole et al. (2004) estimated a Mach number of 1.64 for the merger shocks by applying the Rankine-Hugoniot jump conditions under the assumption that the pre-shock temperature is approximated by the averaged temperature in the central regions of A1750C and $\mathrm{A} 1750 \mathrm{~N}$ and that the post-shock temperature is given by the temperature in the middle region of the two clusters. Belsole et al. (2004) also concluded that A1750C is an unrelaxed cluster for the following four reasons: (1) the discontinuity of the gas density profile in the southeast region (region 3 in their notation), (2) the shift of $\mathrm{X}$-ray centroid from the position of the BCG, (3) the lack of evidence for a cooling flow, and (4) excess entropy in the central region, compared with other relaxed clusters.

No significant offset among distributions of the galaxy luminosity, mass and ICM is found in the binary cluster A1750, as shown in Figure 6.

The X-ray surface brightness peak in A1750C coincide with the position of the BCG (top-right panel) but is offset from the centroid of the X-ray emission, which is consistent with the X-ray analysis by Belsole et al. (2004). The position of the main mass peak $(7.7 \sigma)$ in A1750C coincides with the peak positions of the optical luminosity (bottomleft panel) and the X-ray surface brightness (bottom-right panel), within a smoothing scale (1'25 FWHM). No significant mass substructure is found in A1750C. The observed geometry of mass, galaxies, and X-ray emission in A1750C does not show a signature of recent strong mergers as seen in A754. However, we cannot entirely rule out the possibility of a minor merger of small mass clumps below the weak lensing sensitivity. The averaged X-ray temperature of $\mathrm{A} 1750 \mathrm{C}$ is higher than the temperature predicted by the best-fitting SIS model to the tangential shear profile, as shown in Figure 13 (see also $\$ 4.3$ ). This is consistent with the lack of cool gas and the excess entropy in the central region (Belsole et al. 2004).

A1750N has two BCGs whose positions coincide with that of the X-ray peak of A1750N (top-right panel). The mass peak $(5.9 \sigma)$ of $\mathrm{A} 1750 \mathrm{~N}$ is slightly, but not significantly, offset from the X-ray and galaxy-luminosity peaks, and the offset is within the smoothing scale of FWHM $=1 ! 25$. The temperature $T_{\text {SIS }}$ estimated from weak lensing coincides with the X-ray temperature $T_{X}$ for A1750N (see §4.3).

As seen from the bottom-right panel of Figure 6, the reconstructed mass distribution traces an extended Xray substructure (XM in Figure 6) between A1750N and $\mathrm{A} 1750 \mathrm{C}$, which is around $(\mathrm{RA}, \mathrm{DEC})=\left(202.77^{\circ},-1.765^{\circ}\right)$. Overall, the ICM and galaxy distributions in the binary cluster A1750 and its components, A1750C and A1750N, are in good agreement with the mass distribution.

\section{3. $A 1758$}

A1758 is a binary cluster at $z=0.2790$. The ROSAT observation by Rizza et al. (1998) showed that this cluster consists of two clusters (A1758N and A1758S) separated by $\sim 8^{\prime}$ on the sky, corresponding to the projected physical separation of $\sim 2 \mathrm{Mpc}$. David \& Kempner (2004) found from the XMM-Newton observation that the line-of-sight velocity difference between A1758N and A1758S is less than $\Delta V=2100 \mathrm{kms}^{-1}$. David \& Kempner (2004) argued that their physical proximity to each other and small velocity difference are consistent with those of a gravitationally interacting system. The Chandra and XMM-Newton observations showed that there is no X-ray signature of the interaction between A1758N and A1758S (David \& Kempner 2004), contrary to the case of the binary cluster A1750 which has a high temperature region between the two components (see $§ 3.2$ ). This system is most likely in an earlier merger stage than A1750, and therefore serves as an ideal target for studying the cluster mass distribution in the initial stage of the merging process. As shown in Figure 7, the XMM-Newton X-ray image shows a bridge connecting between the two cluster components. On the other hand, the reconstructed mass map shows no significant mass structure corresponding to the bridge in the X-ray image. Note that the mass structure located on the X-ray bridge is a local minimum ( $\mathrm{M}$ in Figure 7 ). It is also shown in Figure 7 that there is no significant offset between the mass structures and the X-ray/optical structures of A1758N and A1758S along the north-south direction connecting between A1758N and A1758S, which is consistent with results for A1750 (§3.2).

The system components, A1758N and A1758S, are both undergoing mergers (David \& Kempner 2004). David \& Kempner (2004) found from XMM-Newton data average gas temperatures of $\mathrm{A} 1758 \mathrm{~N}$ and $\mathrm{A} 1758 \mathrm{~S}$ to be $8.2 \pm 0.4$ $\mathrm{keV}$ and $6.4_{-0.4}^{+0.3} \mathrm{keV}$, respectively, at the $90 \%$ confidence level. Using Chandra data, David \& Kempner (2004) obtained an average gas temperature of A1758N to be $9.0_{+0.9}^{-0.6} \mathrm{keV}$ at the $90 \%$ confidence level. For A1758N, the XMM-Newton and Chandra measurements of the average gas temperature are in good agreement with each other.

A1758N has a complex X-ray morphology as seen in the top right panel of Figure 7: there are northwest and southeast X-ray subclumps in the cluster central region. The Chandra X-ray image suggests that the northwest subclump (XC in Figure 7 ) is currently moving towards the north and that the southeast subclump (XSE in Figure 7) is currently moving towards the southeast (David \& Kempner 2004). The optical luminosity distribution (bottom-left panel) reveals two luminous subclumps of cluster-sequence galaxies in A1758N. The Xray peak position of the northwest subclump coincides 
with that of the luminous BCG (top-right panel). The southeast gas subclump is, on the other hand, offset by $\approx 290 \mathrm{kpc}$ to the northwest of the other galaxy subclump (top-right panel).

Figure 7 shows that mass and light are similarly distributed in A1758N. The mass map shows double peaks: the first peak with a significance of $13.5 \sigma$ (SE in Figure 7) corresponding to the southeast luminous galaxy clump, and the second peak with a significance of $11.0 \sigma$ ( $\mathrm{C}$ in Figure 7) corresponding to the northwest luminous galaxy clump (bottom-left panel). A visual inspection also reveals several gravitational arc candidates around the two mass peaks in A1758N, supporting the bimodal mass distribution in A1758N. Figure 15 displays zoom in views of tangential arc candidates in the $R_{\mathrm{c}}$-band image. The tangential arc candidates $\mathrm{A}, \mathrm{B}$, and $\mathrm{C}$ are bluer than the cluster red sequence in $g^{\prime}-R_{\mathrm{c}}$ color. The tangential arc candidates D and $\mathrm{E}$ are associated with cluster galaxy concentrations. This bimodal feature of A1758N in the weak lensing mass map was previously reported by Dahle et al. (2002) based on weak lensing data taken with ALFOSC on Nordic Optical Telescope. We note that Dahle et al. (2002) also found an arc-like image in A1758N.

The northwest galaxy clump has a higher luminosity than the southeast one. The angular extent of the northwest galaxy clump is larger than that of the southeast one. These optical features would suggest that the northwest mass structure $(\mathrm{C})$ is the primary component of $\mathrm{A} 1758 \mathrm{~N}$ and the southwest clump (SE) is the merging substructure. $N$-body simulations of $\Lambda \mathrm{CDM}$ models show that weak lensing measurements of cluster mass peaks have a large scatter $(\sim 100 \%$ for less massive systems with $\left.M_{200} \sim 10^{14} M_{\odot} h^{-1}\right)$ due to line-of-sight projection effects of intervening mass structures as well as due to intrinsic ellipticities of background galaxies (White, van Waerbeke \& Mackey 2002; Wu et al. 2006). Therefore the relative peak heights of the mass peaks SE and C $(13.5 \sigma$ and $11.0 \sigma$ significance, respectively) could have been substantially affected by such effects. The southeast structure in the mass/galaxy map is located in front of the X-ray southeast subclump moving towards the southeast. This geometry regarding the southeast substructure is consistent with the results from the joint optical photometric, X-ray, and weak lensing analysis of the merging cluster 1E0657-56 (Clowe, Gonzale, \& Markevitch 2004). On the other hand, the northwest structure, which is likely to be the main component of A1758N, shows no significant offset among the galaxy, ICM, and mass distributions, which is different from the results of 1E0657-56.

A1758S has an elongated X-ray emitting core located close to the first peak in the galaxy luminosity distribution (right panels). David \& Kempner (2004) pointed out that the X-ray core has two bow-like shaped edges which are curved up toward north and south, respectively. They suggested that these features are a signature of the merger along a line between the northwest and southeast sides with a small impact parameter. No optical counterpart has been detected in the Subaru image (left panels), whereas two luminous galaxy concentrations are found along the northeast-southwest direction, which is perpendicular to the hypothetical merging direction. The second optical peak associated with A1758S is offset from the X-ray core to the southwest by $\sim 480 \mathrm{kpc}$. The high density region with $\kappa>9 \sigma$ significance is elongated along the northeast-southwest direction, aligned with the two luminous galaxy concentrations. The mass peak associated with A1758S is located in the middle of the two optical clumps, and has a peak height of $9.9 \sigma$. The angular separation between the two luminous subclumps is comparable to the smoothing scale FWHM $=1.25$ for the weak lensing reconstruction. At this smoothing scale, we cannot therefore resolve possible mass substructures originally associated with the optical subclumps. Nonetheless, the elongated mass and X-ray structures and the existence of two luminous galaxy concentrations indicate that A1758S is not yet in dynamical equilibrium and is an ongoing merger. The merger geometry of A1758S is puzzling, because the curved-up directions of the X-ray core are almost perpendicular to both directions of the elongations of the mass and light distributions. If the observed direction of the elongation in mass and light is regarded as the original collision axis, then our results would indicate that not all arc-like cores in the X-ray emission can be used to trace the path of the moving core.

\section{4. $A 1914$}

The cluster A1914 is an on-going merger at $z=0.1712$ with averaged temperature of $10.9 \pm 0.7 \mathrm{keV}$ at $90 \%$ confidence (Govoni et al. 2004). A1914 has two X-ray cores embedded in a round X-ray halo: As shown in the topright panel of Figure 8 (see also Govoni et al. 2004), the larger X-ray core (XE in Figure 8) is elongated in the eastwest direction, and the smaller core (XW in Figure 8) is located to the west of the larger core. Based on Chandra X-ray data, Govoni et al. (2004) found a filamentary hot region along the northeast-southwest direction connecting between the two small cores, and argued that the filamentary hot region is a signature of the shock-heated gas induced by the elongated small X-ray core moving towards the west.

We have obtained for A1914 a high resolution mass map with a Gaussian FWHM of 0.75 as shown in Figure 8 (left panels). The cluster mass distribution is highly irregular, whereas the Chandra X-ray map shows a nearly circular morphology except the central core region (top-right panel). We found seven mass concentrations above a detection threshold of $4 \sigma$ significance in the $\kappa$ map covering a $16^{\prime} \times 15^{\prime} .5$ field around A1914. There are two prominent mass peaks with $\kappa \gtrsim 0.3$ : The first mass peak (C1 in Figure 8) has a peak height of $9.1 \sigma$, and its position is in good agreement with the second brightest cluster galaxy (BCG2 in Figure 8) which is close to the elongated Xray core. The position of the second mass peak with a significance of $7.8 \sigma$ (C2 in Figure 8), on the other hand, coincides well with that of the brightest cluster galaxy (BCG1 in Figure 8) sitting in the optical center. The observed geometry of the galaxy and mass distributions would suggest that the second mass peak (C2) associated 
with the optical cluster center (BCG1) is the primary cluster center, and the first mass peak $(\mathrm{C} 1)$ is the merging substructure. By visual inspection we found an arc-like image tangentially-oriented with respect to $\mathrm{BCG} 2 / \mathrm{C} 1$, as pointed out by Dahle et al. (2002) and Sand et al. (2005). The curvature of this tangential arc candidate is consistent with the existence of the high density peak (C1) around BCG2 (see Figure 16).

However, it remains puzzling that the projected mass distribution in A1914 is so irregular contrary to the quasi circular X-ray distribution in the outer region. A detailed analysis will be presented in a forthcoming paper (Umetsu \& Okabe in preparation).

\subsection{A2034}

A2034 is a cold front cluster at $z=0.1130$ well studied with ROSAT, ASCA and Chandra X-ray satellites (David, Forman, \& Jones 1999; White 2000; Kempner \& Sarazin 2003). Based on the Chandra observation, Kempner \& Sarazin (2003) found a sharp discontinuity in the X-ray surface brightness $\sim 3^{\prime}$ to the north of the cluster center, similar to the surface brightness jump associated with cold fronts. The X-ray morphology suggests that this dense core is moving toward the north direction. Kempner \& Sarazin (2003) showed that the temperature is fairly constant to a radius of at least about $5^{\prime}$ from the center of the main cluster in A2034, and hence no significant temperature jump, associated with the dense core, is found. They also argued that the excess emission from the southern part of the dense core is likely to be a background structure. Kempner \& Sarazin (2001) found evidence for the existence of a radio relic near the cold front.

The resulting mass distribution in A2034 is highly irregular and quite different from the X-ray surface brightness distribution of the XMM-Newton data as shown in Figure 9. In the central region of this cluster $\left(\sim 18 .^{\prime} 5 \times 18 .^{\prime} 5\right)$, there are six significant mass concentrations with peak heights greater than $4 \sigma$ significance (left panels). The main mass peak with a significance of $6.4 \sigma$ ( $\mathrm{C}$ in Figure 9) coincides with the first peak in the X-ray surface brightness distribution (see the bottom-right panel of Figure 9). There are two BCGs close to the first X-ray and mass peaks, corresponding to the main cluster of A2034.

The second mass peak with a significance of $6.3 \sigma(\mathrm{S}$ in Figure 9) is located to the south of the main mass peak, and coincides with the south excess X-ray emission discovered by Kempner \& Sarazin (2003). No spectroscopic data are available in this region. The reconstructed mass map reveals another three mass structures (W1, W2 and W3 in Figure 9) associated without Xray counterparts in the west of the second mass peak. We confirmed that they are background structures at $z \sim 0.116-0.118$ from SDSS spectroscopic data. The velocity difference between the main cluster and the west structures is $c \delta z \sim 900-1500 \mathrm{kms}^{-1}$, and the comoving radial separation is $\mathcal{O}(10 \mathrm{Mpc})$ by neglecting their proper motions. Their apparent proximity suggests that the cluster A2034 and the west mass structures could be part of a filamentary structure.
The primary result of our joint analysis on A2034 is the detection of the northern mass subclump with a significance of $5.4 \sigma$ ( $\mathrm{N}$ in Figure 9) located ahead of the north cold front shown in the top-right panel. The second brightest member galaxy of A2034 is located close to the center of the north mass subclump (left panels). This merger geometry is consistent with the results of other cold front clusters, A2142 (§3.6), A1758N (§3.3), and 1E0657-56 (Clowe, Gonzale, \& Markevitch 2004).

\subsection{A2142}

A2142 is a cold front cluster at $z=0.0909$. Cold fronts were discovered for the first time in this cluster by the Chandra X-ray observation (Markevitch et al. 2000). There are two cold fronts in the central region of A2142, as indicated in the top-right panel of Figure 10: One is at the northwest edge (Cold Front NW) of a large core, and the other at the south edge (Cold Front S) of a small core in the X-ray surface brightness distribution. The small core is apparently contained within the large core in the sky plane. Those edges indicate that the large and small cores are moving toward the northwest and the south directions, respectively. The temperatures change abruptly across the cold fronts by a factor of two. There are two BCGs (BCG1 and BCG2) in the cluster core region, as indicated in the top-right panel of Figure 10: BCG1 is located inside the small core, and BCG2 is apparently located in the large core. The two BCGs have a large line-of-sight velocity difference of $1840 \mathrm{kms}^{-1}$ (Oegerle, Hill, \& Fitchett 1995).

Okabe, Umetsu \& Hattori (2008) have obtained a mass map with low angular resolution of FWHM = 3..3 using a different set of Subaru weak lensing data taken under bad weather conditions. Okabe et al. (2008) found that the mass distribution is similar to the luminosity distribution of spectroscopically selected bright cluster member galaxies, but is quite different from the ICM distribution. Okabe et al. (2008), based on the joint Xray/optical/weak-lensing analysis, found a marginal detection $(2.1 \sigma)$ of a northwest mass substructure, which is located ahead of the northwest cold front. The projected separation between the northwest cold front and mass substructure is $\sim 540 \mathrm{kpc}$.

In the present study we have derived a high resolution mass map with FWHM =1.00, as shown in the left panels of Figure 10, using a new Subaru data set with long exposure.

The number density of background galaxies usable for a weak lensing analysis is increased by a factor of $\sim 3$ as compared with the previous data set (Okabe et al. 2008).

Using the new high-resolution mass map, this northwest mass structure (NW in the top-left panel of Figure 10) has been detected at a significance of $\kappa=3.0 \sigma$, which is located in the same region as the previous detection. This improved detection using an independent data set confirms our previous tentative detection, and supports the existence of the northwest mass substructure located ahead of the northwest cold front. In the bottom-left panel of Figure 10, we see a slight excess luminosity of clus- 
ter sequence galaxies associated with the northwest mass substructure, as found earlier by Okabe et al. (2008) using the spectroscopically selected sample of bright cluster galaxies. The Chandra X-ray image shows no X-ray counterpart to the northwest mass substructure (right panels of Figure 10). XMM-Newton observations also show no substructure in the X-ray emission associated with the northwest mass substructure (Okabe et al. 2008). The merger geometry of A2142, that is the existence of mass substructures in front of cold fronts, is similar to other cold front clusters, such as A2034 (§3.5) and 1E0657-56 (Clowe, Gonzale, \& Markevitch 2004).

We have also discovered a mass concentration associated with a luminous galaxy concentration located in front of the south cold front. This south mass substructure (S in Figure 10) has a peak height of $4.3 \sigma$ in the $\kappa$ map, and is located around $(\mathrm{RA}, \mathrm{DEC})=\left(239.54^{\circ}, 27.18^{\circ}\right)$. The line connecting between BCG1 and this south substructure is roughly parallel to the moving direction of the south cold front.

The new high resolution mass map has revealed more complex and detailed structures in A2142 than the lowresolution mass map by Okabe et al. (2008). The significance of the first mass peak is $10.9 \sigma$ in the $\kappa$ map, and the peak position is close to BCG1. We note the presence of two tangential arc candidates around BCG1 as shown in panels A and B of Figure 17.

The line-of-sight velocity (LOSV) of BCG1 is close to the central value (mode) of the LOSV distribution of cluster galaxies (Oegerle, Hill, \& Fitchett 1995). The X-ray, optical, and mass peak positions of the main cluster are in good agreement with each other within FWHM $=1$ '.00 of the weak lensing reconstruction. On the other hand, no significant clumpy mass structure is seen around BCG2. Further we did not find any tangential arc candidate around BCG2. We show in panel $\mathrm{C}$ of Figure 17 a zoom in view of the $0.8 \times 0.8$ region around BCG2. The projected separation between BCG1 and BCG2 is larger than the angular resolution of the mass map, FWHM = 1.'00. BCG2 has a large peculiar velocity, and is in the highvelocity tail of the LOSV distribution in the cluster restframe (Okabe et al. 2008).

There are few member galaxies associated with BCG2 in the LOSV distribution. The mass distribution around BCG2 is peculiar and different from all other BCGs in our target clusters, which are sitting at high density regions with large $\kappa$-values. If BCG2 was originally associated with the northwest mass substructure, then the results may suggest the possibility that BCG and the dark-matter halo underwent different dynamical processes, which then resulted in a large offset between the BCG and the darkmatter halo after the merger.

\subsection{A520}

Markevitch et al. (2005) discovered with Chandra Xray observations a bow-shaped shock close to a dense gas clump in the cluster A520 at $z=0.199$. They derived a Mach number of $M=2.1_{-0.3}^{+0.4}$ with the Rankine-Hugoniot condition. The post and pre-shock temperatures are found to be $11.5_{-3.1}^{+6.7} \mathrm{keV}$ and $4.8_{-0.8}^{+1.2} \mathrm{keV}$, respectively. The $\mathrm{X}$ ray feature is very similar to the bullet cluster 1E0657-56 (Markevitch et al. 2005), in that a shock detached from a dense core is prominently observed. Therefore this cluster is a good target for a weak lensing study of 'bullet'-type merging clusters.

Two compact gas clumps (XSW1 and XSW2 in Figure 11) are located in the southwest of the cluster center. The gas clump XSW2 associated with the bow shock is close to but slightly offset from a luminous BCG. The extended tail gas from XSW2 is also seen in Figure 11. Markevitch et al. (2005) argued that XSW2 is moving along the northeast-southwest direction. Govoni et al. (2004) found that the temperature is higher along this direction based on Chandra data. On the other hand, the gas clump XSW1 is elongated in the east-west direction, and its X-ray contours are compressed towards the northwest direction (see the right panels of Figure 11), suggesting that XSW1 is currently moving towards the northwest direction. The presence of two gas clumps might suggest that A520 is a three-component merger.

Our weak lensing analysis of A520 is based on archival $i^{\prime}$-band data retrieved from SMOKA. We have three $i^{\prime}$ imaging data sets used for our weak lensing analysis as described in $\S 2.1$. Here we shall focus on the analysis results using the deepest data set (see Table 1), which is based on the same data used by Mahdavi et al. (2007). A comparison of mass maps with the three different data sets is devoted in Appendix 2.

We show in the left panels of Figure 11 contour plots of the reconstructed mass distribution in A520. The smoothing scale for the weak lensing mass reconstruction is $\mathrm{FWHM}=1 ! 25$. The first mass peak (C1 in Figure 11) is located in the southeast of the optical cluster center, and the detection signal-to-noise ratio is $6.1 \sigma$. The mass peak $\mathrm{C} 1$ is located $\sim 0.5$ east of the central $\mathrm{X}$-ray surface brightness peak (XC in Figure 11). Recently Mahdavi et al. (2007) reported the presence of a massive dark core (the mass peak 3 of Mahdavi et al. 2007) located $\sim 0$.'7 south of the central X-ray peak, XC; the offset between the dark core and $\mathrm{XC}$ is smaller than the angular resolution of Mahdavi et al.'s mass map $\left(\mathrm{FWHM}=1^{\prime}\right)$. The first peak $\mathrm{C} 1$ in our mass map and the dark core of Mahdavi et al. (2007) coincide with XC within Gaussian FWHMs of our and their mass maps, and the projected distance between $\mathrm{C} 1$ and the dark core is about 1!.0. In the central region of the $\kappa$ map, there are two more significant local maxima (C2 and C3 in Figure 11): C2 with a significance of $5.8 \sigma$ is located in the north of $\mathrm{C} 1$, and $\mathrm{C} 3$ with a significance of $5.4 \sigma$ in the east of $\mathrm{C} 1$. The locations of $\mathrm{C} 2$ and C3 agree well with those of the main and a local maxima of the optical luminosity density distribution (see the bottom-left panel of Figure 11), respectively. In the central high-density region of A520, the shape of mass contours with $\kappa \gtrsim 4 \sigma$ significance is similar to that of the cluster galaxy luminosity distribution.

We note that although Mahdavi et al. (2007) used the same Subaru/Suprime-Cam $i^{\prime}$ data as in the present paper, their analysis method is different from ours in the fol- 
lowing way: Our weak lensing analysis is based on three sets of stacked Subaru/Suprime $i^{\prime}$ data (see Appendix 2), while Mahdavi et al. (2007) performed shape measurements separately for each of seven Subaru exposures and combined individual weak lensing catalogs to improve the accuracy for the shear estimates. We have confirmed the existence of the dark core $(\mathrm{C} 1)$ reported by Mahdavi et al. (2007) in two of the imaging data sets; however, the peak C1 is not particularly pronounced in the mass map based solely on $i^{\prime}$ images taken without AG. We have detected C3 at high significance levels ( $\gtrsim 4.5 \sigma$ ) from all of the three imaging data sets (see Appendix 2), while Mahdavi et al. (2007) found no significant mass structure around C3,

The second mass peak (SW2 in Figure 11) with a significance of $6.0 \sigma$ is associated with the southwest substructure. In the southwest region, mass and light are similarly distributed, and the peak positions in the mass and luminosity maps coincide well with each other (see the bottom-left panel of Figure 11). On the other hand, the $\mathrm{X}$-ray surface brightness peak associated with the southwest X-ray core (XSW2 in Figure 11) is slightly offset from the corresponding galaxy-luminosity and mass peaks; the separation between the X-ray and mass peaks is smaller than the Gaussian smoothing scale of FWHM $=1$ '.25 used for the weak lensing mass reconstruction. The southwest compact gas, XSW2, seems to be within the potential well of the southwest mass substructure, SW2. We also find a local mass peak with a significance of $3.5 \sigma$ (SW1 in Figure 11) located ahead of the northwest of the gas clump XSW1. The mass peak SW1 is associated with a concentration of cluster member galaxies (see the bottomleft panel of Figure 11). The projected separation between SW1 and the northwest edge of XSW1 is about 1!.6.

We note that, in addition to these mass peaks, the reconstructed $\kappa$ map shows three more local maxima above a significance of $4 \sigma$, which are indicated as N, NE1, and NE2 in Figure 11: the mass clumps NE2 and N are associated with slight concentrations of cluster member galaxies. On the other hand, there is no apparent concentration of galaxies having similar $\left(V-i^{\prime}\right)$ colors around the location of NE1, although this mass structure is seen all of the three data sets (see Appendix 2).

Finally, the results from our joint optical, X-ray, and weak-lensing analysis show that although the X-ray features of A520 are very similar to those of the bullet cluster 1E0657-56, the relative positions of the galaxy, X-ray, and mass substructures are different, in that there is no apparent offset between the mass (SW2) and the gas (XSW2) clumps associated with the bow shock.

\section{GLOBAL CLUSTER PROPERTIES}

We present and compare global cluster properties, such as the total mass, optical luminosity, and X-ray temperature, for our sample of merging clusters based on the joint optical-photometric/X-ray/weak-lensing analysis.

\subsection{Cluster Mass}

We show in Figure 12 radial profiles of the projected mass $M_{\zeta}(\theta)$ for our sample of seven merging clusters together with the best-fitting NFW and SIS profiles. Note that the error bars are correlated; in the model fitting, we take into account the full error covariance matrix for the $M_{\zeta}$ measurements. The projected mass $M_{\zeta}(\theta)$ is measured using aperture densitometry, $\zeta_{c}$, by $M_{\zeta}(<\theta) \equiv$ $\pi\left(D_{d} \theta\right)^{2} \Sigma_{\mathrm{cr}} \zeta_{c}\left(\theta ; \theta_{\text {inn }}, \theta_{\text {out }}\right)$ (see equation [15] and $\left.\S 2.3 .4\right)$. Table 7 summarizes the best-fitting SIS and NFW model parameters derived for our sample of merging clusters. For each target and its cluster component (if exists) we chose the position of the BCG as the cluster center for measuring global cluster properties. The $\theta_{\text {inn }}$ and $\theta_{\text {out }}$ define the inner and outer boundaries of the background annulus for the $\zeta_{c}$-statistic measurement. The values of $\left(\theta_{\text {inn }}, \theta_{\text {out }}\right)$ are chosen so as to avoid cluster binary components and/or significant background substructures, and they are summarized in Table 8 . The radial range used for the fit is different for each cluster: the upper limit, $\theta_{\max }$, is set to the maximum radius which will avoid contamination by neighboring substructures and foreground/background mass structures; the lower limit, $\theta_{\min }$, is set so as to avoid the strong-lensing regime (see Table 7 ). As we have seen in $\S 3$, the mass distribution in merging clusters is highly irregular and complex. Indeed, when fitting NFW/SIS models to the $\zeta_{c}$-statistic measurements, we found for some clusters large $\chi^{2}$-values relative to the degrees-offreedom (dof). For all of the cluster targets, the virial mass $M_{\text {vir }}$ of the best-fitting NFW model is consistent with that of the SIS model (see $\S 2.3 .4$ ) within $1 \sigma$ statistical error. On the other hand, the NFW concentration parameter $c_{\text {vir }}$ was poorly constrained, except for a marginal detection of A2142 $(\approx 2.5 \sigma)$, because of contamination by neighboring substructures and clusters in projection space.

As defined by equation (15), $M_{\zeta}(<\theta)$ yields a lower bound to the true enclosed mass $M(<\theta)$ due to the subtraction of the mean $\kappa$ over the background annulus, $\bar{\kappa}\left(\theta_{\text {inn }}<\theta<\theta_{\text {out }}\right)$. In Table 8 we summarize the results of weak-lensing total mass estimates, $M_{\zeta}\left(<\theta_{M}\right)$, for our sample of merging clusters. The aperture radius $\theta_{M}$ is set to the maximum radius available for each target cluster, i.e. $\theta_{M} \approx \theta_{\max }$.

\subsection{Mass-to-light Ratio}

We measure for individual targets the cluster luminosity from color-selected member galaxies (see §2.2). The cluster luminosity is measured in $R_{\mathrm{c}}$-band for all clusters, except in $i^{\prime}$-band for A520. Here the aperture and the background annulus for each target are taken to be the same as in aperture densitometry. Table 8 lists the mass-to-light $(M / L)$ ratio derived for our sample of merging clusters, where the errors include a systematic uncertainty in the background luminosity density estimation as well as $1 \sigma$ random uncertainties in the cluster luminosity and mass measurements; the lower limit on the cluster $M / L$ ratio is based on the cluster luminosity without background 
subtraction and $1 \sigma$ random uncertainties for the $M / L$ ratio. The clusters A754 and A1750 have considerably high $M / L$ ratios of $M / L \gtrsim 600 h(M / L)_{\odot}$, which is simply due to the fact that cluster galaxies are still dominating in the background annular region and hence the background luminosity density is overestimated.

Girardi et al. (2000) studied optical properties of a sample of 105 nearby Abell-ACO clusters $(z<0.15)$ for which virial mass estimates are available, and found typical values for $M / L_{B_{j}}$ ratios to be $230-250 h\left(M / L_{B_{j}}\right)_{\odot}$. Sanderson \& Ponman (2003) conducted a joint optical/Xray study of the mass composite of a sample of 66 relaxed groups/clusters. Based on the X-ray mass measurements, they obtained a logarithmic mean value of $M / L_{B_{j}} \approx$ $350 h(M / L)_{\odot}$, which is scaled to $M / L_{R_{\mathrm{c}}} \approx 190 h\left(M / L_{R_{\mathrm{c}}}\right)_{\odot}$ assuming $\left(L / L_{\odot}\right)_{B_{j}}=0.547\left(L / L_{\odot}\right)_{R_{\mathrm{c}}}$ from a typical color $\left(B_{j}-R\right)=1.702$ of early-type galaxies (equation [4] of Sanderson \& Ponman 2003).

Clowe et al. (2006) performed a weak lensing analysis of 20 high- $z$ clusters $(z>0.4)$ selected from the ESO Distant Cluster Survey (EDisCS) using deep 3-color optical images taken with the VLT/FORS2. They derived for an individual cluster $M / L$ ratios, in $I$ and $B$ passbands, within $500 \mathrm{kpc}$ from photo- $z$ selected cluster galaxies. They found for their optically-selected EDisCS clusters that the clusters tend to have a lower $M / L$ ratio at higher redshifts, but found no change in their $M / L$ ratios with cluster mass. Clowe et al. (2006) also showed that the clusters having additional structures in projection space have a higher measured $M / L$ ratio, which is probably due to the projection effects.

\subsection{X-ray vs. SIS Temperatures}

We compare for individual cluster targets the X-ray temperature, $T_{X}$, and the best-fitting SIS temperature, $T_{\mathrm{SIS}}$, obtained from the aperture densitometry measurements. In Table 8 we summarize the best-fitting SIS velocity dispersion, $\sigma_{v}$, from the weak lensing analysis. The $\mathrm{X}$-ray temperatures of the clusters are taken from the literature (see Table 2). We note there is a good agreement between the Chandra and XMM-Newton temperatures of A1758N (David \& Kempner 2004), although Chandra and XMM-Newton have different instrument responses as well as sky and instrumental background levels. The X-ray temperature measurement is performed within apertures of $\theta \sim 10^{\prime}$, while the weak lensing measurement of aperture densitometry within apertures of $\theta_{M} \sim 5^{\prime}-15^{\prime}$ (see Table 8), depending on the availability of the clean background aperture without any significant mass structures. We emphasize that the SIS temperature $T_{\text {SIS }}$ based on gravitational lensing does not depend on the dynamical and physical state of the cluster system.

Figure 13 compares the X-ray temperature and the SIS temperature for our sample of merging clusters. It is shown in Figure 13 that there is an overall trend that the ICM temperatures from the X-ray observations are higher than the SIS temperature from the weak lensing analysis. Figure 14 shows explicitly the X-ray to SIS temperature ratio, $T_{X} / T_{S I S}$, as a function of $T_{\mathrm{SIS}}$. The sample mean for $T_{X} / T_{\mathrm{SIS}}$ is estimated as $\left\langle T_{X} / T_{\mathrm{SIS}}\right\rangle=2.05 \pm 0.11$.

For A1750N, where mass, light, and X-ray emission are similarly distributed (see $\S 3.2$ ), the temperature ratio $T_{X} / T_{\text {SIS }}$ is consistent with unity within the $1 \sigma$ error bar. The ICM temperature of A1750C, which shows an excess entropy in the central region, is higher than $T_{\text {SIS }}$ (§3.2), but is consistent with $T_{\mathrm{SIS}}$ within $2 \sigma: T_{X} / T_{\mathrm{SIS}}=$ $1.72 \pm 0.53(1 \sigma)$. The averaged X-ray temperature of A $520, T_{X}=7.1 \pm 0.54 \mathrm{keV}(1 \sigma)$, is slightly higher than the SIS temperature, $T_{\mathrm{SIS}}=5.9 \pm 1.1 \mathrm{keV}(1 \sigma)$, but is marginally consistent within $1 \sigma$ with $T_{\mathrm{SIS}}$ : $T_{X} / T_{\mathrm{SIS}}=$ $1.18 \pm 0.25(1 \sigma)$; However, we note that the post-shock temperature of A520, $T_{X}=11.5_{-3.1}^{+6.7} \mathrm{keV}(90 \% \mathrm{CL})$, is significantly higher than $T_{\text {SIS }}$, while its pre-shock temperature, $T_{X}=4.8_{-0.8}^{+1.2} \mathrm{keV}(90 \% \mathrm{CL})$, is marginally consistent with $T_{\mathrm{SIS}}$.

For the rest of the sample (A754, A1758N, A1758S, A1914, A2034, A2142) consisting of on-going mergers and cold-front clusters, the ratios $T_{X} / T_{\text {SIS }}$ are in the range of $1.4-3.1$, with the sample mean of $\left\langle T_{X} / T_{\mathrm{SIS}}\right\rangle=2.41 \pm 0.16$. Hence, presumably the most promising scenario is that the ICM temperature is increased by heating processes triggered by cluster mergers. On the other hand, the temperature ratios for the cold front clusters, A2034 and A2142, are in a moderate range of $1.94 \pm 0.38$ and $1.41 \pm 0.19$, respectively.

Although the averaged temperatures $T_{X}$ of the coldfront clusters were measured including the cold, dense cores, the X-ray temperatures outside the dense cores agree with the global temperatures (including the cold cores) within errors (Markevitch et al. 2000; Kempner $\&$ Sarazin 2003). Therefore, the observed variations and trends in the $T_{X} / T_{\text {SIS }}$ ratios would be naturally explained by the different energy scales injected into the ICM or different merger states of the cluster mergers. Figure 14 shows no clear correlation between the temperature ratio $T_{X} / T_{\text {SIS }}$ and the SIS temperature $T_{\text {SIS }}$. This might suggest that the enhancement of the ICM temperature does not depend strongly on the mass of the main cluster but on merging stages and conditions.

\section{DISCUSSION AND CONCLUSIONS}

We presented and compared weak lensing mass, optical light, and X-ray emission maps for a sample of seven merging clusters of galaxies. We have selected for the present study seven nearby Abell clusters $(0.0542 \leq z \leq 0.279)$ of different merging stages and properties, and conducted systematic, deep imaging observations of the seven target clusters with Suprime-Cam on Subaru telescope. Our seven target clusters, representing various merging stages and conditions, allow us to investigate in details the physical interplay between dark matter, ICM, and galaxies associated with hierarchical structure formation. The clusters A1750 and A1758 are binary systems, each of which consists of two cluster-sized components and has not yet experienced the first impact. A520, A754, A1758N, A1758S, and A1914, on the other hand, are classified as on-going merging clusters. A2034 and A2142 are "cold 
front" clusters.

In the binary cluster A1750, the projected mass, optical light, and X-ray distributions all revealed clear binary structures associated with the two cluster components (A1750N and A1750C), and are overall similar and regular without significant substructures (§3.2). A1758 also exhibits binary structures associated with the two system components (A1758N and A1758S) in the projected mass, optical light, and X-ray distributions (§3.3). Along the line between A1758S and A1758N, which is parallel to the dynamical motion of the binary components, no significant offset is found between the mass, X-ray, and optical peaks in the binary components. On the other hand, the ICM distribution in individual components, each of which is an undergoing merger, appears to be different from the distributions of mass (dark matter) and light (galaxies). Our results on the binary clusters indicate that, in an early merging phase, the distributions of mass and baryons are overall similar and the main mass peak of the cluster coincides well with the corresponding X-ray peak and galaxy concentration.

The mass distributions of on-going merging clusters, A520, A754, A1758N, A1758S, and A1914, are found to be highly irregular. Overall, the mass distribution appears to be similar to the galaxy luminosity distribution, whereas their distributions are totally different from the ICM distribution. Discrepancies between mass and ICM distributions are different in a variety of ways. Our results on the on-going mergers can be formally classified into three types depending on the moving direction and relative positions of mass and X-ray substructures. Here we speculate on the moving direction of cool, dense cores from the direction of compression and elongation of X-ray cores and trails, and summarize the results as follows:

(1) No significant offset between X-ray and mass peaks

The mass peak position coincides with the peak position of X-ray surface brightness within Gaussian smoothing FWHM of the weak lensing mass reconstruction. This apparent feature would imply that these dense cores are trapped in the gravitational potential well of the mass concentration. This is found in the southwest clump (SW2 and XSW2) of A520, the northwest clump (C and XC) of $\mathrm{A} 1758 \mathrm{~N}$, and the first mass peak and the elongated gas core (C1 and XE) of A1914. The position of the BCG in A1758N coincides with the peak position of the dense core in the ICM (XC). For the case of A520 and A1914, the associated luminous galaxy is slightly offset perpendicular to the moving direction of the gas core.

(2) Mass clump in front of the dense core

The second type is the case where the mass clump is located ahead of the moving gas core, as seen in the southwest structure (SE and XSE) in A1758N. The bullet cluster 1E0657-56 is categorized into this type. We note that this configuration is also found in our cold front clusters, A2034 (N and the cold front) and A2142 (NW and S), as we will discuss below. The following scenario could explain this type of merger configurations: a dense gas core, which is originally bound in a merging substructure, is stripped away from the mass and galaxy components of the substructure by the ram pressure of the ICM.

\section{(3) Mass clump behind the dense core}

The third type is where the mass clump is located behind the dense core, which is opposite to the second case. This is found in the east substructure (E and XE) of A754. A possible explanation is that this cluster is in a merging phase right after reaching its apocenter of the merger orbit (see §3.1), although no direct evidence from currently available data.

Cluster mergers depend on a certain set of merging parameters, such as the mass ratio of the main to sub cluster, initial velocity and angular momentum of the merging substructure, and the shape of the gravitational potential of the main cluster. Furthermore, clusters are in various phases of the merging process. Understanding and constraining the dynamical process that causes offsets between the mass/ICM/galaxy components therefore require a more detailed, quantitative study of these merged substructures, which will be presented elsewhere. For example, line-of-sight kinematic information of member galaxies from spectroscopic observations, measurements of the density, temperature, and entropy of the pre/postshock regions will be valuable.

The mass maps of the cold front clusters A2034 and A2142 reveal irregular mass structures, which are quite different from the ICM distribution. Mass structures with low gas mass fractions have been detected in front of three cold fronts, which is found earlier in the bullet cluster 1E0657-56 (Clowe et al. 2004). Such merger configurations are classified as the second type of on-going mergers as discussed above. To date four cold fronts have been studied via joint X-ray/weak-lensing analysis including 1E0657-56, and all of the cold fronts show such a configuration of mass/cold-front substructures. This might be thus a common feature of cold front clusters associated with the formation of cold fronts. Current results from the joint analysis of cold front clusters suggest a possible scenario for the formation of cold fronts that a substructure falls into and passes through the primary cluster halo, while the hot gas originally bound in the substructure is stripped away by ram pressure stripping (see Clowe et al. 2004). A more detailed, statistical study of the relationship between cold fronts and associated mass structures is required to draw more definitive conclusions on the formation of cold fronts.

The observed positions of dense cores in A2034 and A2142 are apparently located in the primary cluster halo in projection space. This is different from the case of the bullet cluster 1E0657-56, which shows a significant offset between the mass and X-ray halos of the primary cluster as well as between those of the subcluster (Clowe et al. 2004). However, since no line-of-sight information of substructures is available from the current data sets, the merger geometry in three-dimensional space has not yet been fully constrained. Such line-of-sight information of the primary and sub clusters will be necessary to place 
quantitative constraints on the evolution of the gas temperature and pressure of the ICM substructure against the gravitational potential of the primary cluster.

Our quantitative comparison of projected distributions of mass, galaxies, and ICM in merging clusters shows that the dark matter and ICM components exhibit different behaviors during the cluster merger process. Similar features have been reported based on cosmological $N$ body/hydrodynamical simulations. Tormen, Moscardini, \& Yoshida (2004) have shown by their numerical simulations that dark matter halos of merged substructures freely move and oscillate in the primary cluster halo, while gas halos initially bound in the substructure are stripped by various physical processes, such as ram pressure stripping and dynamical friction. These behaviors depend on the mass ratio between the primary and sub cluster. It is easier for dark matter of small substructures to survive longer, while for the ICM of small substructures to be destroyed or digested by the primary cluster. They also showed that the separation between the dark matter and gas centers for a merged substructure, whose mass is more than 0.01 times of the primary cluster mass, is very small in the early phase of $\sim 1$ Gyr. These simulation results are consistent with our results of the binary cluster A1750 (§3.2). Takizawa (2006) studied the X-ray and mass distributions of 1E0657-56 based on $N$-body/hydrodynamical simulations, and reproduced a clear offset between X-ray and mass peak as found from the joint X-ray/weak-lensing analysis by Clowe et al. (2004). The mass ratio of the sub to main halo is assumed to be $1 / 16$, which is based on the weak lensing mass estimate by Clowe et al. (2004). Takizawa (2006) also derived an analytic expression for the ram pressure stripping conditions of the substructure in mergers of two NFW dark matter halos, which is useful to understand the merging conditions of the first and second types defined above. Ricker \& Sarazin (2001) and Mathis, Lavaux, Diego, \& Silk (2005) studied cluster mergers of nearly-equal-mass with numerical simulations and showed that the hot gas of a merged substructure escapes from the local gravitational potential well when it reaches the apocenter in merger orbits, because the inward motion of the gas is delayed compared with the associated dark matter clump. Their simulation results would be a clue to understand the third type of on-going mergers as found in A754. Nagai \& Kravtsov (2003) searched for cold fronts and their counterparts in their high-resolution cluster simulations, and found cold fronts appear to be common in major and minor cluster mergers in hierarchical models. Their results indicate cold fronts are non-equilibrium transient phenomena during cluster mergers, although no significant offset between dark matter and gas distributions is shown in their figure. Ascasibar \& Markevitch (2006) used high-resolution simulations of cluster mergers and showed that cold fronts in relaxed clusters are due to sloshing of the cool gas in the gravitational potential of the primary cluster, and can be easily triggered by minor mergers and persist for gigayears. Based on three-dimensional hydrodynamic simulations Takizawa (2005) investigated the dynamical evo- lution of the ICM driven by a radially-moving substructure and its observational implications, and found that the subcluster's cold gas is pushed out of its potential well around the turn around, resulting in that the cold gas clump appears to be in front of the main X-ray peak of the subcluster (see Figure 6 of Takizawa 2005).

A comparison of the ICM and SIS temperatures of merging clusters from X-ray and weak-lensing analyses, respectively, provides us with interesting pieces of evolutionary information of the ICM during the cluster merger. Our results show that the ICM temperature in on-going and cold-front clusters is significantly higher than the SIS temperature that is a measure of the virial temperature of the cluster (Figure 13). This would suggest that the ICM temperature is increased by the cluster merger. Similar results were found from numerical simulations. Randall, Sarazin, \& Ricker (2002) and Rowley, Thomas, \& Kay (2004) studied the effects of merger boosts on the ICM properties and showed that cluster mergers can boost for a duration of the sound crossing time the X-ray luminosity and temperature of the merged cluster above the virial equilibrium values for the merged system. Our results show no significant correlation between the SIS temperature, or the cluster mass, and the X-ray to SIS temperature ratio, $T_{X} / T_{\mathrm{SIS}}$; this might indicate that the energy release from cluster mergers is determined not only by the cluster mass but also by other merging parameters. Ricker \& Sarazin (2001) studied offset mergers between clusters based on $N$-body/hydrodynamic simulations as a function of impact parameter and mass ratio of the colliding clusters, and show that the variations in global properties of clusters, namely total luminosity and average temperature, indeed depend on the impact parameter and the mass ratio, as well as the observed epoch of mergers.

Based on visual inspection, we have identified gravitational arc candidates in three of our cluster targets: A1758, A1914, and A2142 (see Figures 15, 16, and 17). A further examination and confirmation of the lensing hypothesis of these gravitational arc candidates will require follow up spectroscopy and higher-resolution imaging, which will provide strong lensing constraints needed for a detailed modeling of the observed arc-cluster systems.

In summary we compared projected distributions of mass and baryons in a sample of seven merging clusters based on the joint optical-photometric/X-ray/weaklensing analysis. The global cluster parameters, such as the cluster mass, the cluster mass-to-light ratio, and the ICM temperature, are derived for individual targets of our cluster sample.

A joint analysis of optical photometric, X-ray, and weak lensing data provides us with a comprehensive picture of cluster mergers, which will help to better understand the complex physical interplay between dark matter, ICM, and galaxies in the cluster formation process. For merging clusters an X-ray analysis alone cannot constrain the cluster mass distribution since the ICM is not in hydrostatic equilibrium. We have demonstrated that weak lensing is indeed a unique and powerful method to map the 
mass distribution in merging clusters that are in the process of active evolution and formation, since it does not require any assumption of the physical/dynamical state of the system. Furthermore, thanks to the Subaru/SuprimeCam with excellent image quality and wide-field imaging capability, we were able to achieve high-resolution and wide-field imaging in weak lensing mass reconstructions. In particular, it is worth noting that the Subaru/SuprimeCam makes it possible to reveal a mass distribution of a very nearby cluster at $z \sim 0.05$ (A754), where the lensing signal is very low due to its proximity to the observer. A detailed study of individual targets using the joint data sets is the next step for understanding the merger dynamics of clusters and associated physical processes of the ICM. To date galaxy clusters have been studied extensively and systematically in X-ray and optical photometric observations. On the other hand, the weak-lensing database for clusters is not sufficiently large as compared with X-ray and optical, although much progress has been made in the recent years (e.g., Dahle et al. 2002; Cypriano et al. 2004; Bardeau et al. 2005, 2007; Clowe et al. 2006; Hoekstra 2007).

Therefore a systematic weak-lensing study of a larger sample of clusters needs to be conducted for further understanding of clusters and their formation/evolution process.

\section{Acknowledgments}

This work is in part supported by Grants-in-Aid for the 21st Century COE Program "Exploring New Science by Bridging Particle-Matter Hierarchy" in Tohoku University and "Towards a New Basic Science; Depth and Synthesis" in Osaka University, funded by the Ministry of Education, Science, Sports and Culture of Japan. The work is partially supported by the National Science Council of Taiwan under the grant NSC95-2112-M-001-074-MY2. This work is in part supported by a Grant-in-Aid for Science Research in a Priority Area "Probing the Dark Energy through an Extremely Wide and Deep Survey with Subaru Telescope" (18072001) from the Ministry of Education, Culture, Sports, Science, and Technology of Japan .

We gratefully thank H. Furusawa, the Subaru Support Astronomer of the Suprime-Cam. We acknowledge fruitful and intensive discussions with M. Takada, H. Hoekstra, T. Broadhurst, T. T. Takeuchi, M. Takizawa, M. Hattori, N. Ota, S. S. Sasaki, T. Nagao, and Y. Fujita. We are also grateful to N. Kaiser for developing the IMCAT package publicly available.

\section{Appendix 1. Lensing Mass Models}

The SIS density profile is given by

$$
\rho_{\mathrm{SIS}}(r)=\frac{\sigma_{v}^{2}}{2 \pi G r^{2}}
$$

where $\sigma_{v}$ is the one-dimensional velocity dispersion of the SIS halo. The $\sigma_{v}$ is related with the virialization epoch $z_{\text {vir }}$ and the virial mass $M_{\mathrm{vir}}$ of the SIS halo as

$$
\begin{aligned}
\sigma_{v}\left(M_{\mathrm{vir}}, z_{\mathrm{vir}}\right) & =\frac{1}{2} r_{\mathrm{vir}} H_{0} \sqrt{\Omega_{m 0} \Delta_{\mathrm{vir}}\left(1+z_{\mathrm{vir}}\right)^{3}}, \\
M_{\mathrm{vir}} & =\frac{4 \pi}{3} \bar{\rho}\left(z_{\mathrm{vir}}\right) \Delta_{\mathrm{vir}} r_{\mathrm{vir}}^{3}
\end{aligned}
$$

where $r_{\mathrm{vir}}=r_{\mathrm{vir}}\left(M_{\mathrm{vir}}, z_{\mathrm{vir}}\right)$ is the virial radius, and $\Delta_{\mathrm{vir}}$ is the mean overdensity with respect to the mean cosmic density $\bar{\rho}\left(z_{\text {vir }}\right)=\Omega_{m}\left(z_{\text {vir }}\right) \rho_{\mathrm{cr}}\left(z_{\text {vir }}\right)$ at the virialization epoch, predicted by the dissipationless spherical tophat collapse model (Peeebles 1980; Eke, Cole, \& Frenk 1996; Bullock et al. 2001). We assume the cluster redshift $z$ is equal to the cluster virial redshift $z_{\text {vir }}$. We use the following fitting formula in a flat 3-space with cosmological constant (see Oguri, Taruya, Suto 2001):

$$
\Delta_{\text {vir }}=18 \pi^{2}\left(1+0.4093 \omega_{\text {vir }}^{0.9052}\right) \text {, }
$$

where $\omega_{\text {vir }} \equiv 1 / \Omega_{m}\left(z_{\text {vir }}\right)-1$.

We thus have $\sigma_{v} \propto M_{\mathrm{vir}}^{1 / 3}$. In the case of an SIS lens, the lensing convergence is obtained as

$$
\kappa_{\mathrm{SIS}}(\theta)=\frac{\theta_{E}}{2 \theta}
$$

where $\theta_{E}=4 \pi\left(\sigma_{v} / c\right)^{2}\left(D_{d s} / D_{s}\right)$ is the Einstein radius. Then the averaged convergence within $\theta$ is

$$
\bar{\kappa}_{\mathrm{SIS}}(<\theta)=\frac{\theta_{E}}{\theta}=2 \kappa_{\mathrm{SIS}}(\theta) .
$$

The NFW universal density profile has a two-parameter functional form as

$$
\rho_{\mathrm{NFW}}(r)=\frac{\rho_{s}}{\left(r / r_{s}\right)\left(1+r / r_{s}\right)^{2}}
$$

where $\rho_{s}$ is a characteristic inner density, and $r_{s}$ is a characteristic inner radius. In stead of using $r_{s}$, we introduce the concentration parameter, $c_{\text {vir }} \equiv r_{\text {vir }} / r_{s}$. The inner density $\rho_{s}$ can be expressed in terms of other virial properties of the NFW halo:

$$
\rho_{s}=\bar{\rho}\left(z_{\mathrm{vir}}\right) \frac{\Delta_{\mathrm{vir}}}{3} \frac{c_{\mathrm{vir}}^{3}}{\ln \left(1+c_{\mathrm{vir}}\right)-c_{\mathrm{vir}} /\left(1+c_{\mathrm{vir}}\right)}
$$

$\rho_{\mathrm{cr}}$ is the critical density of the universe at the cluster redshift $z_{d}$, and $r_{s}$ is the NFW scaling radius. Hence, for a given cosmological model and a halo virial redshift $\left(z_{\text {vir }}\right)$, we can specify the NFW model with the halo virial mass $M_{\mathrm{vir}}$ and the halo concentration parameter $c_{\mathrm{vir}}$.

For an NFW profile, it is useful to decompose the convergence $\kappa(\theta)$ and the averaged convergence $\bar{\kappa}(<\theta)$ as

$$
\begin{aligned}
\kappa_{\mathrm{NFW}}(x) & =\frac{b}{2} f(x), \\
\bar{\kappa}_{\mathrm{NFW}}(<x) & =\frac{b}{x} g(x),
\end{aligned}
$$

where $b=4 \rho_{s} r_{s} / \Sigma_{\text {cr }}$ is the dimensionless scaling convergence, $x=\theta /\left(r_{s} / D_{d}\right)$ is the dimensionless angular radius, and $f(x)$ and $g(x)$ are dimensionless functions. We have analytic expressions for $f(x)$ and $g(x)$ as (Bartelmann 1996): 


$$
\begin{aligned}
& f(x)=\left\{\begin{array}{cc}
\frac{1}{1-x^{2}}\left[-1+\frac{2}{\sqrt{1-x^{2}}} \operatorname{arctanh} \sqrt{\frac{1-x}{1+x}}\right. & (x<1) \\
\frac{1}{3} \quad(x=1) & (\text { A } 11) \\
\frac{1}{x^{2}-1}\left[+1-\frac{2}{\sqrt{x^{2}-1}} \arctan \sqrt{\frac{x-1}{x+1}}\right. & (x>1) .
\end{array}\right. \\
& g(x)= \begin{cases}\frac{2}{x \sqrt{1-x^{2}}} \operatorname{arctanh} \sqrt{\frac{1-x}{1+x}}+\frac{1}{x} \ln \frac{x}{2} & (x<1) \\
1-\ln 2 & (x=1)(\mathrm{A} 12) \\
\frac{2}{x \sqrt{x^{2}-1}} \arctan \sqrt{\frac{x-1}{x+1}}+\frac{1}{x} \ln \frac{x}{2} & (x>1)\end{cases}
\end{aligned}
$$

Finally, we use the following identity to calculate the theoretical $\zeta_{c}$-statistic for a given set of the aperture parameter $\left(\theta_{\text {inn }}, \theta_{\text {out }}\right)$ :

$$
\begin{aligned}
\zeta_{c}(\theta) \equiv & \bar{\kappa}(<\theta)-\bar{\kappa}\left(\theta_{\text {inn }}<\theta<\theta_{\text {out }}\right) \\
= & \bar{\kappa}(<\theta)-\bar{\kappa}\left(<\theta_{\text {inn }}\right) \\
& +\frac{1}{1-\left(\theta_{\text {inn }} / \theta_{\text {out }}\right)^{2}}\left(\bar{\kappa}\left(<\theta_{\text {inn }}\right)-\bar{\kappa}\left(<\theta_{\text {out }}\right)\right) .
\end{aligned}
$$

In practice, we use a discretized estimator for equation (15). We note that, unlike tangential shear measurements, measurement errors for $\zeta_{c}(\theta)$ are correlated between different annular bins.

Appendix 2. Comparison of Weak Lensing Mass Reconstructions between Three Different Data Sets for A520

We retrieved from the Subaru Archival Website, SMOKA, a total of seven $i^{\prime}$-band images of A520 taken under good seeing conditions $\left(\sim 0 .^{\prime \prime} 6\right)$. The archival $i^{\prime}$ data of A520 were taken with and without AG (acquisition and guide) probe on 17th November 2001 and 19th October 2001, respectively. The use of AG probe is required for accurate telescope pointing, which is crucial for weak lensing shape measurements of faint background galaxies. We performed a weak lensing analysis separately on the following three imaging data sets: (A) all of seven $i^{\prime}$ images taken both with and without guide probe $(7 \times 240$ s exposure) used in the weak lensing analysis of Mahdavi et al. (2007), (B) four $i^{\prime}$ images taken without guide probe $(4 \times 240$ s exposures $)$, and $(\mathrm{C})$ three $i^{\prime}$ images taken with guide probe $(3 \times 240$ s exposure). Therefore, $(B)$ and (C) are independent and taken under different observing modes, while (A) is a combination of (B) and (C). We note that the A520 data were taken with large dithering offsets of $\approx 2 ! 3$, whereas the other cluster data were taken with a dithering offset of $1^{\prime}$.

Our weak lensing analysis is based on co-added mosaics of multiple exposures (A), (B), and (C), whereas Mahdavi et al. (2007) performed shape measurements separately for each of seven Subaru exposures and combined individual weak lensing catalogs to improve the accuracy for the shear estimates. In the present paper, the deepest data set (A) is used for our main analysis of A520, and the resulting mass map is compared with X-ray and opticalluminosity distributions of the cluster in Figure 11. Figure 18 , on the other hand, compares the mass reconstruction based on (B) with the X-ray and optical-luminosity distributions.
We show in Figure 19 the mass maps of A520 reconstructed from the above three imaging data sets. The left panel in Figure 19 shows the mas map from the deepest data set (A), which is used for the main analysis of the present paper (see also Figure 11); the middle panel shows the mass map from (B) taken without guide probe; the right panel shows the mass map from $(\mathrm{C})$ taken with guide probe. For all of the reconstruction, the Gaussian smoothing FWHM is taken to be 1!25. The contours are spaced in units of $1 \sigma$ reconstruction error of each data set.

The primary mass peak $\mathrm{C} 1$ found from the data set (A) is also detected at a significance of $5.6 \sigma$ from the data set (C) taken with guide probe. The angular position of $\mathrm{C} 1$ agrees with that of the dark core discovered by Mahdavi et al. (2007) within Gaussian FWHM used for the mapmaking. However, this mass clump is not particularly pronounced in the mass map obtained from (B).

The mass clump C3 of (A) is associated with a local concentration of cluster member galaxies (see Figure 11). The mass clump C3 is found to be the first peak in the reconstructed mass map from (B). The mass structure around C3 is not strongly pronounced in the mass map based on (C), but the reconstruction shows a clear feature of the extending mass structure towards the southeast direction, which is seen in the optical luminosity map of the cluster (see the bottom-left panel of Figure 11). However, we note Mahdavi et al. (2007) found no significant mass structure around C3 from their multi-telescope, multi-bandpass weak lensing analysis based on Subaru/Suprime-Cam $R_{\mathrm{c}}$ and $i^{\prime}$ and CFHT/MegaCam $r^{\prime}$ data.

The mass clump $\mathrm{C} 2$ of $(\mathrm{A})$ corresponds to the primary peak in the optical luminosity distribution of the cluster. The mass reconstruction based on (B) shows a local maximum around $\mathrm{C} 2$, whereas no local maximum is found around $\mathrm{C} 2$ in the mass map from $(\mathrm{C})$.

The mass structures SW2, NE1, and NE2 are seen in all of the three reconstructions above a significance level of $4 \sigma$. The mass clump SW1 is detected at a significance level of $3.5 \sigma$ from $(\mathrm{A})$, and is marginally detected at $\approx 3 \sigma$ significance from (B), but is not seen in the mass reconstruction from $(\mathrm{C})$.

Details of mass structures are different in a qualitative and quantitative way between the results from (B) and (C) taken without and with guide probe, respectively. This difference in detailed mass structures might be due to the different pointing modes and exposures between the data sets (B) and (C), which could affect the shape measurements of faint background galaxies. Furthermore, the large dithering offsets of $2 ! 3$ could also make it difficult to correct for the PSF anisotropy in stacked images, as discussed by Mahdavi et al. (2007). Careful follow-up imaging observations with guide probe and small dithering offsets $\left(\sim 1^{\prime}\right)$ will make it possible to clarify these discrepancies in detailed mass structures in A520.

\section{References}

Abell, G. O., Neyman, J., \& Scott, E. L., AJ, 69, 529. Ascasibar, Y. \& Markevitch, M. 2006, ApJ, 650, 102. 
Arnaud, M., et al. 2002, A\&A, 390, 27.

Ashman, K., M., Bird, C., M. \& Zepf, S., E., 1994, AJ, 108, 2348 .

Bardeau, S., Kneib, J.-P., Czoske, O., Soucail, G., Smail, I., Ebeling, H. \& Smith, G. P. 2005, A\&A, 434, 433.

Bardeau, S., Soucail, G., Kneib, J. P., Czoske, O., Ebeling, H., Hudelot, P., Smail, I. \& Smith, G. P. 2007, A\&A, 470, 449.

Bartelmann 1996, A\&A, 313, 697

Bartelmann, M. \& Schneider, P., 2001, PhR, 340, 291.

Baier, F. W. \& Ziener, R., AN, 298, 87.

Belsole, E., Pratt, G. W., Sauvageot, J.-L. \& Bourdin, H. 2004, 415,821 .

Bertin, E. \& Arnouts, S. 1996, A\&AS, 117, 393.

Bradač, M. et al. 2006, ApJ, 652, 937.

Broadhurst, T., Takada, M., Umetsu, K., Kong, X., Arimoto, N., Chiba, M. \& Futamase, T. 2005, ApJ, 619, L143.

Bruzual, G. \& Charlot, S. 2003, MNRAS, 344,1000.

Bullock, J. S., Kolatt, T. S., Sigad, Y., Somerville, R. S., Kravtsov, A. V., Klypin, A. A., Primack, J. R. \& Dekel, A., 2001, MNRAS, 321, 559.

Clowe, D., Luppino, G. A., Kaiser, N., \& Gioia, I. M., 2000, ApJ, 539, 540.

Clowe, D., Gonzalez, A. \& Markevitch, M. 2004, ApJ, 604, 596.

Clowe, D. et al. 2006, A\&A, 451, 395.

Cypriano, E. S., Sodre, L., Jr., Kneib, J.-P. \& Campusano, L. E. 2004, ApJ, 613, 95.

Dahle, H., Kaiser, N., Irgens, R. J., Lilje, P. B. \& Maddox, S. J. 2002, ApJS, 139, 313.

David, L. P., Forman, W. \& Jones, C. 1999, ApJ, 519, 533.

David, L. P. \& Kempner, J. 2004, ApJ, 613, 831.

Donnelly, R. H., Forman, W., Jones, C., Quintana, H., Ramirez, A., Churazov, E.\& Gilfanov, M. 2001, ApJ, 562, 254

Eke, V. R., Cole, S. \& Frenk, C. S. 1996, MNRAS, 282, 263.

Erben, T., van Waerbeke, L., Bertin, E., Mellier, Y., \& Schneider, P. 2001, A\&A, 366, 717

Fahlman, G., Kaiser, N., Squires G. \& Woods, D. 1994, ApJ, $437,56$.

Finoguenov, A., Böhringer, H. \& Zhang, Y.-Y., 2005, A\&A, $442,827$.

Forman, W., Bechtold, J., Blair, W., Giacconi, R., van Speybroeck, L. \& Jones, C. 1981, ApJ, 243, L133.

Geller, M. J. \& Beers, T. C., PASP, 94, 421.

Girardi, M., Borgani, S., Giuricin, G., Mardirossian, F. \& Mezzetti, M., 2000, ApJ, 530, 62.

Goto, T. et al. 2002, PASJ, 54, 515.

Govoni, F., Markevitch, M., Vikhlinin, A., VanSpeybroeck, L., Feretti, L.\& Giovannini, G. 2004, ApJ, 605, 695.

Hamana et al., 2003, ApJ, 597, 98.

Hoekstra, H. 2007, MNRAS, 379, 317.

Henriksen, M. J. \& Markevitch, M. 1996,ApJ, 466,L79.

Henry, J. P. \& Briel, U. G. 1995, ApJ, 443. L9.

Henry, J. P., Finoguenov, A. \& Briel, U. G., 2004, ApJ, 615, 181.

Hudson, M. J., Gwyn, S. D. J., Dahle, H. \& Kaiser, N., 1998, ApJ, 503, 531.

Hetterscheidt, M. et al., 2007, A\&A, 468, 859 (astro$\mathrm{ph} / 0606571)$.

Hoekstra, H., Franx, M., Kuijken, K., \& Squires, G., 1998, ApJ, 504, 636.

Kaiser, N. \& Squires, G. 1993, ApJ, 404, 441.

Kaiser, N. 1995, ApJL, 439, L1.

Kaiser, N., Squires, G., Broadhurst, T., 1995, ApJ, 449, 460.
Katgert, P., Biviano, A. \& Mazure, A. 2004, ApJ, 600, 657.

Kempner, J. C. \& Sarazin, C. L. 2001 ApJ, 548, 639.

Kempner, J. C. \& Sarazin, C. L. 2003 ApJ, 593, 291.

Novicki, M., Jones, C., \& Donnelly, R. H. 1998, AAS, 193, 3807.

Mahdavi, A., Hoekstra, H., Babul, A., Balam, D.\& Capak, P. 2007, ApJ, 668, 806.

Markevitch, M. et al. 2000, ApJ, 541, 542.

Markevitch, M., Gonzalez, A. H., David, L., Vikhlinin, A., Murray, S., Forman, W., Jones, C., \& Tucker, W. 2002, ApJ, 567, L27.

Markevitch, M. et al. 2003, ApJ, 586, L19.

Markevitch, M., Govoni, F., Brunetti, G. \& Jerius, D. 2005, ApJ, 627, 733.

Mathis, H., Lavaux, G., Diego, J. M. \& Silk, J., 2005, MNRAS, $357,801$.

Medezinski, E., Broadhurst, T., Umetsu, K. et al., ApJ, 663, 717

Miyazaki, S. et al. 2002, PASJ, 54, 833,801.

Nagai, D., \& Kravtsov, A. V., 2003, ApJ, 587, 514.

Navarro, J. F.,Frenk, C. S. \& White, S. D. M. 1996, ApJ, 462, 563.

Novicki, M., Jones, C. \& Donnelly, R. H. 1998, AAS, 193, 3807.

Oegerle, W. R., Hill, J. M. \& Fitchett, M. J., 1995, AJ, 110, 32.

Oguri, M., Taruya, A., Suto, Y. 2001, ApJ, 559, 572

Okabe, N., Umetsu, K. \& Hattori, M., in preparation

Ouchi, M., et al. 2004, ApJ, 611, 660.

Peebles, P. J. E., 1980 The large-scale structure of the universe (Princeton University Press)

Randall, S. W., Sarazin, C. L. \& Ricker, P. M. 2002, ApJ, 577, 579.

Ricker, P. M. \& Sarazin, C. L. 2001, ApJ, 561, 621.

Rizza, E., Burns, J. O., Ledlow, M. J., Owen, F. N., Voges, W. \& Bliton, M. 1998,MNRAS, 301, 328.

Rowley, D. R., Thomas, P. A. \& Kay, S. T., 2004, MNRAS, 352,508 .

Sanderson, A. J. R. \& Ponman, T. J., 2003, MNRAS, 345, 1241.

Sand, D. J., Treu, T., Ellis, R. S. \& Smith, G. P. 2005, ApJ, 627,32 .

Sato, J., Umetsu, K., Futamase, T. \& Yamada, T. 2003, ApJ, $582, \mathrm{~L} 67$.

Schechter, P. 1976, ApJ, 203, 297.

Seitz, S. \& Schneider, P., 1995, A\&A, 297, 287.

Seitz, S. \& Schneider, P., 1996, A\&A, 305, 383.

Seitz, S. \& Schneider, 2001, A\&A, 374, 740.

Takizawa, M. 2005, ApJ, 629, 791.

Takizawa, M. 2006, PASJ, 58, 925

Umetsu \& Okabe, in preparation

Umetsu, K., Tada, M., \& Futamase, T. 1999, Prog. Theor. Phys. Suppl., 133, 53

Umetsu, K. \& Broadhurst, T. 2007, submitted to ApJ (arXiv:astro-ph/0712.3441)

Tormen, G., Moscardini, L. \& Yoshida, N., 2004, MNRAS, 350, 1397.

Van Waerbeke et al., 2000A\&A, 358, 30.

White, D. A, 2000, MNRAS, 312, 663.

White, M., Van Waerbeke, L., Mackey, J. 2002, ApJ, 575, 640

Wu, J.-M., Umetsu, K., Chien, C.-H., Chiueh, T. 2006, submitted to ApJ (astro-ph/0607542).

Yagi, M., Kashikawa, N., Sekiguchi, M., Doi, M., Yasuda, N., Shimasaku, K. \& Okamura, S., 2002, AJ, 123, 66. 
Zabludoff, A., I., Geller, M. J., Huchra, J., P. \& Vogeley, M., S., 1993, AJ, 106, 1273.
Table 3. Color-Magnitude relation

\begin{tabular}{lcccc}
\hline \hline $\begin{array}{l}\text { Cluster } \\
(1)\end{array}$ & $\mathrm{a}$ & $\mathrm{b}$ & $\mathrm{c}$ & $\begin{array}{c}L_{\text {tot }} / L_{\text {obs }} \\
(3)\end{array}$ \\
\hline $\mathrm{A} 754$ & -0.00999 & 1.15662 & 0.16 & 1.00103 \\
$\mathrm{~A} 1750$ & -0.02849 & 1.65197 & 0.12 & 1.00268 \\
$\mathrm{~A} 1758$ & -0.11661 & 3.93112 & 0.16 & 1.04095 \\
$\mathrm{~A} 1914$ & -0.04522 & 2.13056 & 0.10 & 1.01238 \\
$\mathrm{~A} 2034$ & -0.02424 & 1.49218 & 0.18 & 1.00483 \\
A2142 & -0.02019 & 1.51493 & 0.11 & 1.00302 \\
A520 & -0.0236 & 1.33718 & 0.12 & 1.00531 \\
\hline
\end{tabular}

Note - Col. (1): Cluster name. Col. (2): Color-magnitude relation parameters of the form: $\mid$ color $-(a \times \operatorname{mag}+b) \mid<c$, where color $=g^{\prime}-R_{\mathrm{c}}$ and $\operatorname{mag}=R_{\mathrm{c}}$ for all targets, except color $=V-i^{\prime}$ and $\operatorname{mag}=i^{\prime}$ for A520. Col. (3): Correction factor of galaxy luminosities due to the luminosity cutoff, $L_{\text {tot }} / L_{\mathrm{obs}}=\Gamma(2-p) / \Gamma\left(2-p, L_{\lim } / L^{*}\right)$.

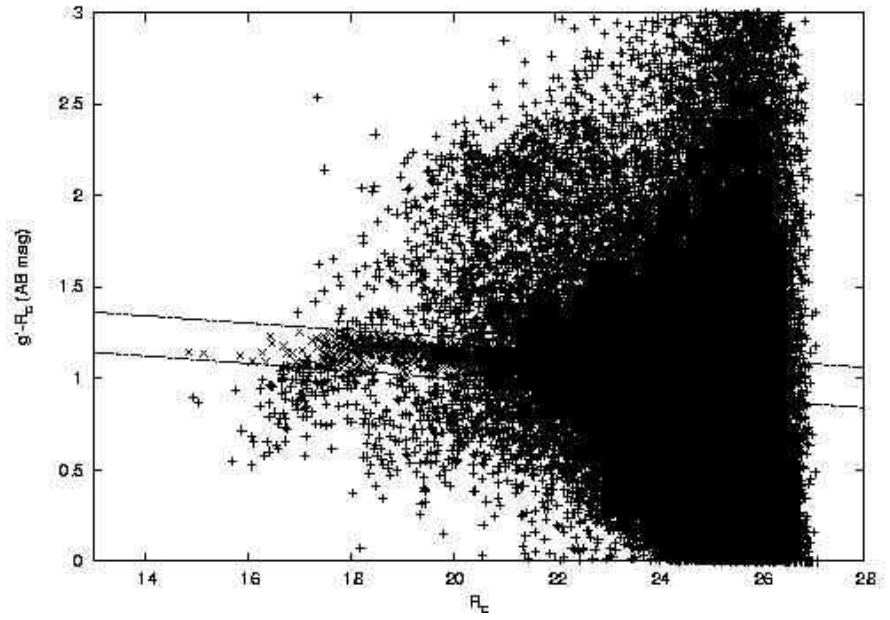

Fig. 1. The color-magnitude diagram of A2142: The earlytype galaxies in clusters are well separated by their colors thanks to the redshifted $4000 \AA$ break. 
Table 1. Target Clusters and Subaru/X-ray Observations

\begin{tabular}{|c|c|c|c|c|c|}
\hline Cluster & Subaru Bands & $\begin{array}{c}\& \text { Exposure Time } \\
(\mathrm{sec})\end{array}$ & $\begin{array}{c}\text { Seeing } \\
(\operatorname{arcsec}) \\
(3)\end{array}$ & $\begin{array}{c}\text { X-ray data } \\
\text { (4) }\end{array}$ & $\begin{array}{c}\text { Obs. ID } \\
(5)\end{array}$ \\
\hline $\mathrm{A} 754$ & $R_{\mathrm{c}}=360 \mathrm{~s} \times 8$ & $g^{\prime}=180 \mathrm{~s} \times 4$ & 0.75 & Chandra & 577 \\
\hline A1750 & $R_{\mathrm{c}}=360 \mathrm{~s} \times 8$ & $g^{\prime}=180 \mathrm{~s} \times 4$ & 0.67 & XMM & 0112240301 \\
\hline A1758 & $R_{\mathrm{c}}=360 \mathrm{~s} \times 8$ & $g^{\prime}=180 \mathrm{~s} \times 4$ & 0.69 & XMM & $0111160101 \& 0142860201$ \\
\hline A1914 & $R_{\mathrm{c}}=360 \mathrm{~s} \times 8$ & $g^{\prime}=180 \mathrm{~s} \times 4$ & 0.61 & Chandra & 3593 \\
\hline A2034 & $R_{\mathrm{c}}=360 \mathrm{~s} \times 8$ & $g^{\prime}=180 \mathrm{~s} \times 4$ & 0.63 & XMM & 0149880101 \\
\hline A 2142 & $R_{\mathrm{c}}=240 \mathrm{~s} \times 4+360 \mathrm{~s} \times 3$ & $g^{\prime}=120 \mathrm{~s} \times 4$ & 0.55 & Chandra & 5005 \\
\hline $\mathrm{A} 520^{\mathrm{a}}$ & $i^{\prime}=240 \mathrm{~s} \times 7$ & $V=450 \mathrm{~s} \times 4+120 \mathrm{~s} \times 3$ & 0.65 & Chandra & 4215 \\
\hline
\end{tabular}

Note - Col. (1): Cluster name. Col. (2): Band name of Subaru/Suprime-Cam and total exposure time in units of s. The former passband is used for the weak lensing analysis. Col. (3): Seeing FWHM in $R_{\mathrm{c}}$ or $i^{\prime}$ band in units of arcsec. Col. (4): X-ray satellite name of archival data. Col. (5): X-ray observation ID number.

${ }^{a}$ Data were collected at Subaru telescope and obtained from the SMOKA science archive. Four $i^{\prime}$-band images were taken without guide probe on 19th October 2001, while the other three $i^{\prime}$ images were taken without guide probe on 17th November 2001.

Table 2. Cluster X-ray Properties

\begin{tabular}{|c|c|c|c|c|c|}
\hline Cluster & $\begin{array}{c}z \\
(2)\end{array}$ & $\begin{array}{c}\text { Type } \\
\text { (3) }\end{array}$ & $\begin{array}{c}1 \text { arcmin } \\
\left(\mathrm{kpc} h_{70}^{-1}\right) \\
(4)\end{array}$ & $\begin{array}{l}\text { Components } \\
\qquad(5)\end{array}$ & $\begin{array}{c}T_{\text {ave }} \\
(\mathrm{keV}) \\
(6)\end{array}$ \\
\hline A754 & 0.0542 & On-going & 63.1 & & $10.0 \pm 0.3^{\mathrm{a}}$ \\
\hline A1750 & 0.0860 & Binary & 96.7 & $\begin{array}{l}\text { A } 1750 \mathrm{C} \\
\mathrm{A} 1750 \mathrm{~N}\end{array}$ & $\begin{array}{l}3.87 \pm 0.10^{b} \\
2.84 \pm 0.12^{b}\end{array}$ \\
\hline A1758 & 0.2790 & Binary & 254.0 & $\begin{array}{l}\mathrm{A} 1758 \mathrm{~N} \\
\mathrm{~A} 1758 \mathrm{~S}\end{array}$ & $\begin{array}{c}8.2 \pm 0.4^{\mathrm{c}} \\
6.4_{-0.4}^{+0.3 \mathrm{c}}\end{array}$ \\
\hline A1914 & 0.1712 & On-going & 174.9 & & $10.9 \pm 0.7^{\mathrm{a}}$ \\
\hline A2034 & 0.1130 & Cold Front & 123.2 & & $7.9 \pm 0.4^{\mathrm{d}}$ \\
\hline A2142 & 0.0909 & Cold Front & 101.7 & & $8.1 \pm 0.4^{\mathrm{e}}$ \\
\hline A520 & 0.1990 & On-going & 197.2 & & $7.1 \pm 0.9^{\mathrm{a}}$ \\
\hline
\end{tabular}

Note - Col. (1): Cluster name. Col. (2): Cluster redshift. Col. (3): Cluster merger type, classified by their X-ray features. 'Binary' represents the system consisting of two clusters separated by the order of Mpc scales. A 'Cold Front' cluster shows the ICM with the presence of a contact discontinuity. 'On-going' is a cluster whose X-ray surface brightness and temperature maps show irregular morphologies. Col. (4): Physical scale in $\mathrm{kpc} / h_{70}$ unit corresponding to $1^{\prime}$ at the cluster redshift. Col. (5): Name of a component of binary cluster. Col. (6): Spatially averaged temperature

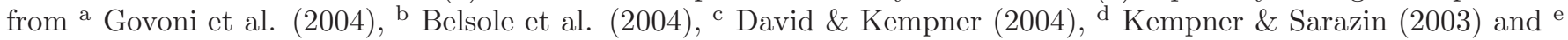
Markevitch et al. (2000). 
Table 4. Weak Lensing Stellar Sample

\begin{tabular}{|c|c|c|c|c|c|c|c|c|}
\hline Cluster & $\begin{array}{r}\text { Uncorrected } e^{*} \\
\bar{e}_{1}^{*} \times 10^{2} \\
(2)\end{array}$ & $\bar{e}_{2}^{*} \times 10^{2}$ & $\sigma\left(e_{*}\right)$ & $\begin{array}{r}\text { Residual } \delta e^{*} \\
\overline{\delta e}_{1}^{*} \times 10^{4} \\
(3)\end{array}$ & ${\overline{\delta e_{2}}}^{*} \times 10^{4}$ & $\sigma\left(\delta e^{*}\right)$ & $\begin{array}{l}N^{*} \\
(4)\end{array}$ & $\begin{array}{r}{\overline{r_{h}}}^{*} \\
\operatorname{arcsec} \\
(5)\end{array}$ \\
\hline A754 & -2.10 & -0.52 & $1.77 \times 10^{-2}$ & $+0.63 \pm 1.30$ & $+0.10 \pm 0.88$ & $4.25 \times 10^{-3}$ & 733 & 0.440 \\
\hline A1750 & +0.65 & -0.05 & $1.73 \times 10^{-2}$ & $-0.46 \pm 1.54$ & $-0.11 \pm 0.92$ & $4.60 \times 10^{-3}$ & 651 & 0.384 \\
\hline A1758 & +0.10 & +0.14 & $1.77 \times 10^{-2}$ & $0.00 \pm 2.44$ & $+0.06 \pm 1.00$ & $4.20 \times 10^{-3}$ & 255 & 0.380 \\
\hline A1914 & -0.85 & +0.50 & $2.25 \times 10^{-2}$ & $+0.42 \pm 2.67$ & $-0.18 \pm 1.68$ & $6.11 \times 10^{-3}$ & 375 & 0.345 \\
\hline A2034 & -1.03 & +2.13 & $2.51 \times 10^{-2}$ & $+0.02 \pm 2.82$ & $-2.70 \pm 2.10$ & $7.52 \times 10^{-3}$ & 458 & 0.342 \\
\hline A2142 & -1.23 & -2.77 & $2.86 \times 10^{-2}$ & $+1.01 \pm 2.64$ & $+4.24 \pm 1.82$ & $8.39 \times 10^{-3}$ & 684 & 0.294 \\
\hline A 520 & -2.40 & -2.43 & $2.98 \times 10^{-2}$ & $+0.19 \pm 1.55$ & $+2.10 \pm 1.29$ & $7.92 \times 10^{-3}$ & 1537 & 0.374 \\
\hline
\end{tabular}

Note - Col. (1): Cluster name. Col. (2): Mean and standard deviation of stellar ellipticities before PSF correction. Col. (3): Mean and standard deviation of stellar ellipticities after PSF correction. Col. (4): Number of stellar objects. Col. (5): Median stellar half-light radius.

Table 5. Background Galaxy Sample and Weak Lensing Mass Reconstruction

\begin{tabular}{lccccc}
\hline \hline Cluster & $\begin{array}{c}\text { Magnitude range } \\
\text { ABmag } \\
(2)\end{array}$ & $\begin{array}{c}n_{g} \\
\operatorname{arcmin}^{-2} \\
(1)\end{array}$ & $\bar{\sigma}_{g}$ & $\begin{array}{c}\text { FWHM } \\
\operatorname{arcmin}\end{array}$ & $\sigma_{\kappa}$ \\
\hline A754 & $19 \lesssim R_{\mathrm{c}} \lesssim 27$ & 37.3 & 0.376 & 1.67 & 0.01739 \\
A1750 & $19 \lesssim R_{\mathrm{c}} \lesssim 27$ & 56.4 & 0.405 & 1.25 & 0.02032 \\
A1758 & $19 \lesssim R_{\mathrm{c}} \lesssim 27$ & 46.4 & 0.408 & 1.25 & 0.02253 \\
A1914 & $19 \lesssim R_{\mathrm{c}} \lesssim 27$ & 47.7 & 0.411 & 0.75 & 0.03737 \\
A2034 & $19 \lesssim R_{\mathrm{c}} \lesssim 27$ & 52.4 & 0.413 & 1.17 & 0.02301 \\
A2142 & $19 \lesssim R_{\mathrm{c}} \lesssim 27$ & 72.1 & 0.395 & 1.00 & 0.02190 \\
A520 & $20 \lesssim i^{\prime} \lesssim 25.5$ & 37.6 & 0.424 & 1.25 & 0.02605 \\
\hline
\end{tabular}

Note - Col. (1): Cluster name. Col, (2): Magnitude range of the background galaxy sample. Col. (3): Surface number density of background galaxies for map-making. Col. (4): RMS error for the shear estimate per galaxy. Col. (5): Gaussian FWHM in map-making. Col. (6): RMS noise in the reconstructed mass map.

Table 6. Red Background Galaxy Sample

\begin{tabular}{lccc}
\hline \hline Cluster & $\begin{array}{c}\text { Magnitude range } \\
\text { ABmag } \\
(1)\end{array}$ & Color & $\begin{array}{c}n_{g} \\
\operatorname{arcmin}^{-2} \\
(1)\end{array}$ \\
\hline A754 & $19 \lesssim R_{\mathrm{c}} \lesssim 27$ & $1.14<g^{\prime}-R_{\mathrm{C}}<15.0$ & $(4)$ \\
A1750 & $19 \lesssim R_{\mathrm{C}} \lesssim 27$ & $1.23<g^{\prime}-R_{\mathrm{C}}<15.0$ & 8.55 \\
A1758 & $19 \lesssim R_{\mathrm{c}} \lesssim 27$ & $1.20<g^{\prime}-R_{\mathrm{C}}<15.0$ & 7.90 \\
A1914 & $19 \lesssim R_{\mathrm{c}} \lesssim 27$ & $1.36<g^{\prime}-R_{\mathrm{c}}<15.0$ & 5.11 \\
A2034 & $19 \lesssim R_{\mathrm{C}} \lesssim 27$ & $1.20<g^{\prime}-R_{\mathrm{C}}<15.0$ & 7.31 \\
A2142 & $19 \lesssim R_{\mathrm{C}} \lesssim 27$ & $1.29<g^{\prime}-R_{\mathrm{C}}<15.0$ & 7.55 \\
A520 & $20 \lesssim i^{\prime} \lesssim 25.5$ & $0.90<V-i^{\prime}<15.0$ & 9.40 \\
\hline
\end{tabular}

Note - Col. (1): Cluster name. Col, (2): Magnitude range of the red background galaxy sample. Col. (3): Color range of the red background galaxy sample. Col. (4): Mean surface number density of the red background galaxies. 
Table 7. Best-Fitting Mass Models

\begin{tabular}{|c|c|c|c|c|c|c|c|}
\hline Cluster & $\begin{array}{c}\mathrm{SIS} \\
\sigma_{v} \\
\left(\mathrm{~km} \mathrm{~s}^{-1}\right) \\
(2)\end{array}$ & $\begin{array}{c}\chi^{2} / \operatorname{dof}(\text { dof }) \\
(3)\end{array}$ & $\begin{array}{c}\left(\theta_{\min }, \theta_{\max }\right) \\
(\operatorname{arcmin}) \\
(4)\end{array}$ & $\begin{array}{c}\text { NFW } \\
M_{\text {vir }} \\
\left(h^{-1} 10^{14} M_{\odot}\right) \\
(5)\end{array}$ & $\begin{array}{l}c_{\mathrm{vir}} \\
(6)\end{array}$ & $\begin{array}{c}\chi^{2} / \operatorname{dof}(\text { dof }) \\
(7)\end{array}$ & $\begin{array}{c}\left(\theta_{\min }, \theta_{\max }\right) \\
(\operatorname{arcmin}) \\
(8)\end{array}$ \\
\hline A754 & $703.1 \pm 139.5$ & $0.32(3)$ & $(6.7,15.0)$ & $2.86 \pm 3.77$ & $4.97 \pm 5.26$ & $2.57(4)$ & $(1.5,5.4)$ \\
\hline A1750N & $633.9 \pm 80.2$ & $1.30(7)$ & $(1.0,7.0)$ & $2.90 \pm 2.08$ & $5.21 \pm 3.42$ & $1.11(6)$ & $(1.0,7.0)$ \\
\hline A $1750 \mathrm{C}$ & $588.9 \pm 90.6$ & $0.91(7)$ & $(1.0,6.7)$ & $1.88 \pm 1.63$ & $5.52 \pm 4.89$ & $0.99(6)$ & $(1.0,6.7)$ \\
\hline $\mathrm{A} 1758 \mathrm{~N}$ & $672.5 \pm 113.3$ & $1.23(4)$ & $(1.7,5.0)$ & $5.26 \pm 5.70$ & $0.24 \pm 0.95$ & $1.01(5)$ & $(1.0,5.0)$ \\
\hline A1758S & $594.5 \pm 94.0$ & $3.51(7)$ & $(0.5,4.6)$ & $1.20 \pm 1.15$ & $3.91 \pm 6.51$ & $1.04(6)$ & $(0.5,4.6)$ \\
\hline A1914 & $845.7 \pm 87.4$ & $1.08(7)$ & $(0.5,5.4)$ & $6.14 \pm 3.19$ & $4.13 \pm 2.79$ & $0.75(6)$ & $(1.0,7.0)$ \\
\hline A2034 & $792.8 \pm 78.2$ & $1.46(10)$ & $(1.0,12.0)$ & $7.17 \pm 4.30$ & $3.74 \pm 2.25$ & $0.84(9)$ & $(1.0,12.0)$ \\
\hline A 2142 & $940.4 \pm 62.6$ & $2.03(10)$ & $(1.0,15.0)$ & $11.95 \pm 5.24$ & $4.32 \pm 1.70$ & $0.83(9)$ & $(1.0,15.0)$ \\
\hline A520 & $960.4 \pm 94.0$ & $0.70(4)$ & $(5.1,15.0)$ & $6.14 \pm 2.38$ & $2.90 \pm 1.82$ & $0.68(7)$ & $(1.7,15.0)$ \\
\hline
\end{tabular}

Note - Col. (1): Cluster name. Col. (2): Best-fitting parameter value for the SIS 1D velocity dispersion. Col. (3): Reduced $\chi^{2}$ for the best-fitting SIS model, and the degrees-of-freedom in parenthesis. Col. (4): Radial range of the $\zeta_{c}$-statistic measurements used for the SIS model fitting. Col. (5): Best-fitting parameter value for the NFW virial mass. Col. (6): Best-fitting parameter value for the NFW concentration parameter. Col. (7): Reduced $\chi^{2}$ for the best-fitting NFW model, and the degrees-of-freedom in parenthesis. Col. (8): Radial range of the $\zeta_{c}$-statistic measurements used for the NFW model fitting.

Table 8. Global Cluster Properties

\begin{tabular}{|c|c|c|c|c|c|}
\hline Cluster & $\begin{array}{c}M_{\zeta}\left(<\theta_{M}\right) \\
\left(h^{-1} 10^{14} M_{\odot}\right) \\
(2)\end{array}$ & $\begin{array}{c}L\left(<\theta_{M}\right) \\
\left(h^{-2} 10^{12} L_{\odot}\right) \\
(3)\end{array}$ & $\begin{array}{c}M_{\zeta} / L\left(<\theta_{M}\right) \\
\left(h M_{\odot} / L_{\odot}\right) \\
(4)\end{array}$ & $\begin{array}{c}\theta_{M} \\
(\operatorname{arcmin}) \\
(5)\end{array}$ & $\begin{array}{c}\left.\theta_{\text {inn }}, \theta_{\text {out }}\right) \\
(\operatorname{arcmin}) \\
(6)\end{array}$ \\
\hline A754 & $2.73 \pm 1.70$ & $0.29^{+0.22}$ & $932^{+635}$ & 15.0 & $(15.0,20.0)$ \\
\hline $\mathrm{A} 1750 \mathrm{~N}$ & $1.49 \pm 0.65$ & $0.22_{-0.01}^{+0.07}$ & $660_{-346}^{+290}$ & 6.96 & $(16.0,21.0)$ \\
\hline $\mathrm{A} 1750 \mathrm{C}$ & $1.26 \pm 0.60$ & $0.17_{-0.01}^{+0.06}$ & $760_{-439}^{+360}$ & 6.65 & $(15.0,20.0)$ \\
\hline A $1758 \mathrm{~N}$ & $4.89 \pm 1.54$ & $2.17_{-0.02}^{+0.13}$ & $225_{-77}^{+71}$ & 5.08 & $(15.0,20.0)$ \\
\hline $\mathrm{A} 1758 \mathrm{~S}$ & $6.06 \pm 1.10$ & $1.20_{-0.02}^{+0.18}$ & $506_{-136}^{+92}$ & 4.63 & $(12.0,17.0)$ \\
\hline A1914 & $4.10 \pm 1.55$ & $1.08_{-0.02}^{+0.22}$ & $380_{-176}^{+140}$ & 6.96 & $(15.0,20.0)$ \\
\hline A2034 & $3.90 \pm 1.81$ & $1.02_{-0.05}^{+0.44}$ & $382_{-229}^{+178}$ & 12.0 & $(12.0,17.0)$ \\
\hline A 2142 & $3.97 \pm 2.04$ & $1.09_{-018}^{+1.37}$ & $365_{-274}^{+197}$ & 15.0 & $(15.0,20.0)$ \\
\hline A520 & $5.18 \pm 4.19$ & $3.40^{+1.35}$ & $152^{+123}$ & 15.0 & $(15.0,20.0)$ \\
\hline
\end{tabular}

Note - Col. (1): Cluster name. Col. (2): Cluster mass $M_{\zeta}\left(<\theta_{M}\right)$ enclosed within $\theta_{M}$ from aperture densitometry. Col. (3): Cluster luminosity enclosed within $\theta_{M}$ in $R_{\mathrm{c}}$-band for all targets, except in $i^{\prime}$-band for A520. Col. (5): Cluster mass-to-light ratio within $\theta_{M}$. Col. (6): Aperture radius in units of arcmin. Col. (8): Inner and outer boundaries of the background annulus for the cluster mass and luminosity measurements, given in units of arcmin. 


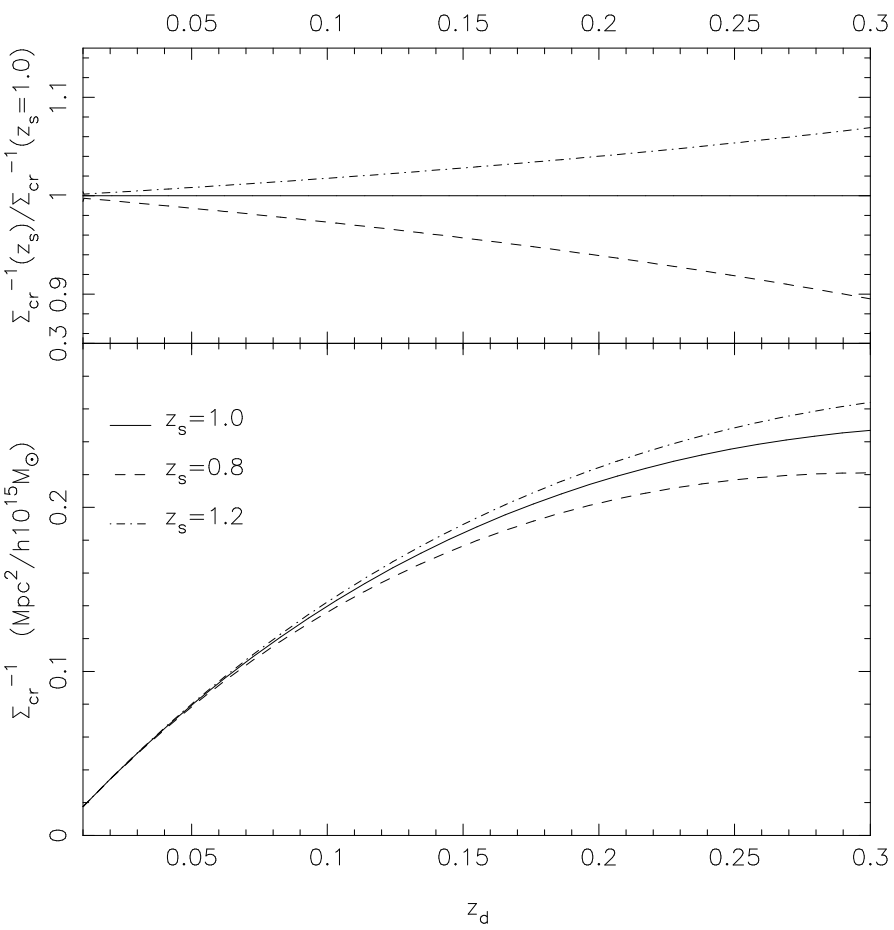

Fig. 2. The lower panel shows the inverse of the critical surface mass density of gravitational lensing, $\Sigma_{\mathrm{cr}}^{-1}\left(z_{d}, z_{s}\right)$, as a function of lens redshift $z_{d}$ for three different source redshifts, $z_{s}=0.8,1.0,1.2$ (dashed, solid, and dotted-dashed, respectively), demonstrating the geometric scaling of gravitational lensing signal. The top panel shows the relative lensing strength $\Sigma_{\mathrm{cr}}^{-1}\left(z_{d}, z_{s}\right) / \Sigma_{\mathrm{cr}}^{-1}\left(z_{d}, z_{s}=1.0\right)$ as a function of lens redshift $z_{d}$ normalized with respect to the source at $z_{s}=1$. For lensing clusters at low redshifts $z_{d}$, $\Sigma_{\mathrm{cr}}$ depends very weakly on the background redshift $z_{s}$, so that the uncertainty in $z_{s}$ of background galaxies is less important in the lensing-based cluster mass determination. 


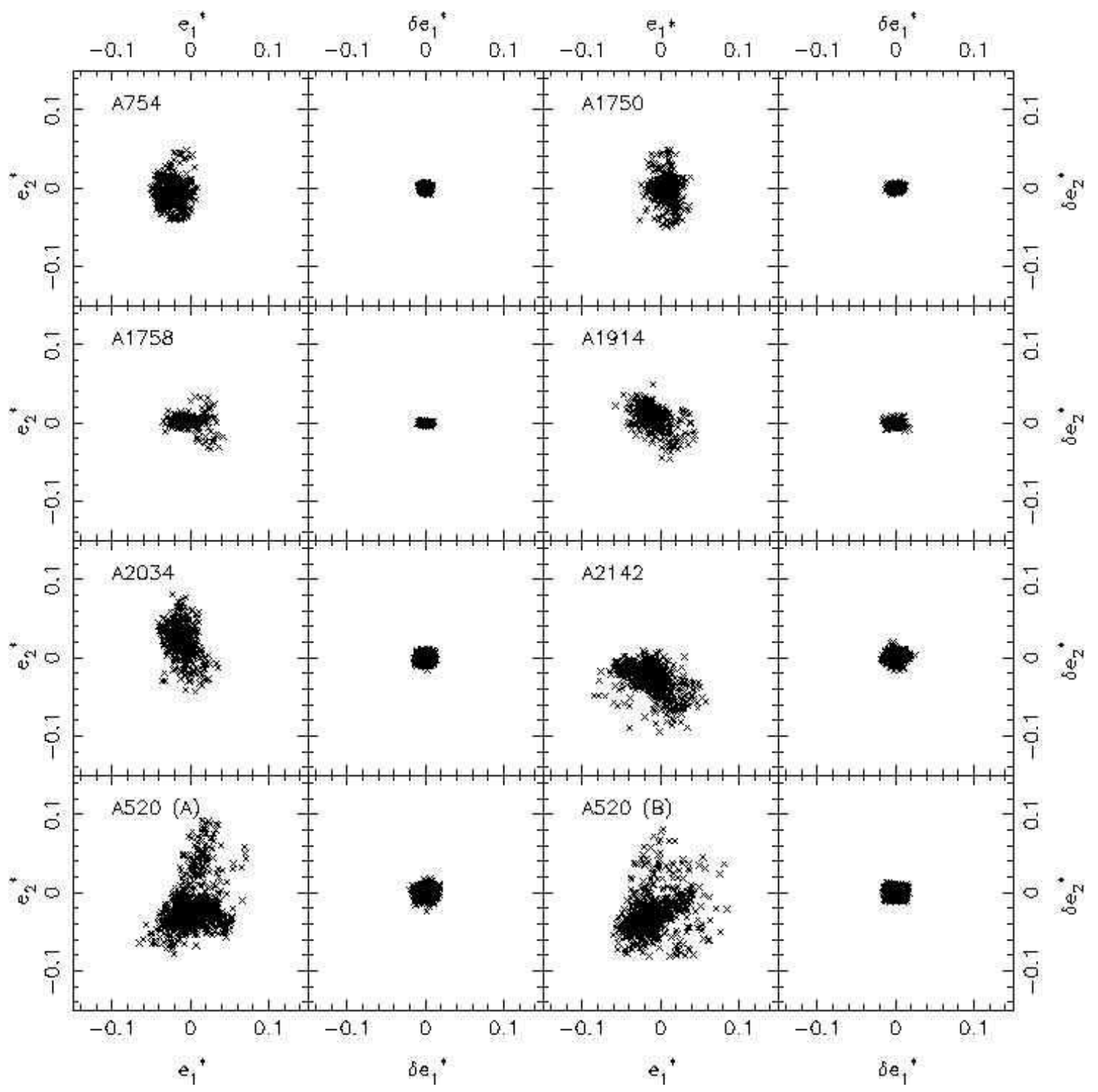

Fig. 3. Stellar ellipticity distributions before and after the PSF anisotropy correction for individual cluster targets. For each cluster the left panel shows the raw ellipticity components $\left(e_{1}^{*}, e_{2}^{*}\right)$ of stellar objects, and the right panel shows the residual ellipticity components $\left(\delta e_{1}^{*}, \delta e_{2}^{*}\right)$ after the PSF anisotropy correction. For A520 two different imaging data sets are shown: A520 (A) is based on a co-added mosaic of seven $i^{\prime}$ images $(7 \times 240 \mathrm{~s})$ taken both with and without guide probe. A520 (B) is based on a co-added mosaic of four $i^{\prime}$ images $(4 \times 240 \mathrm{~s})$ taken without guide probe. 


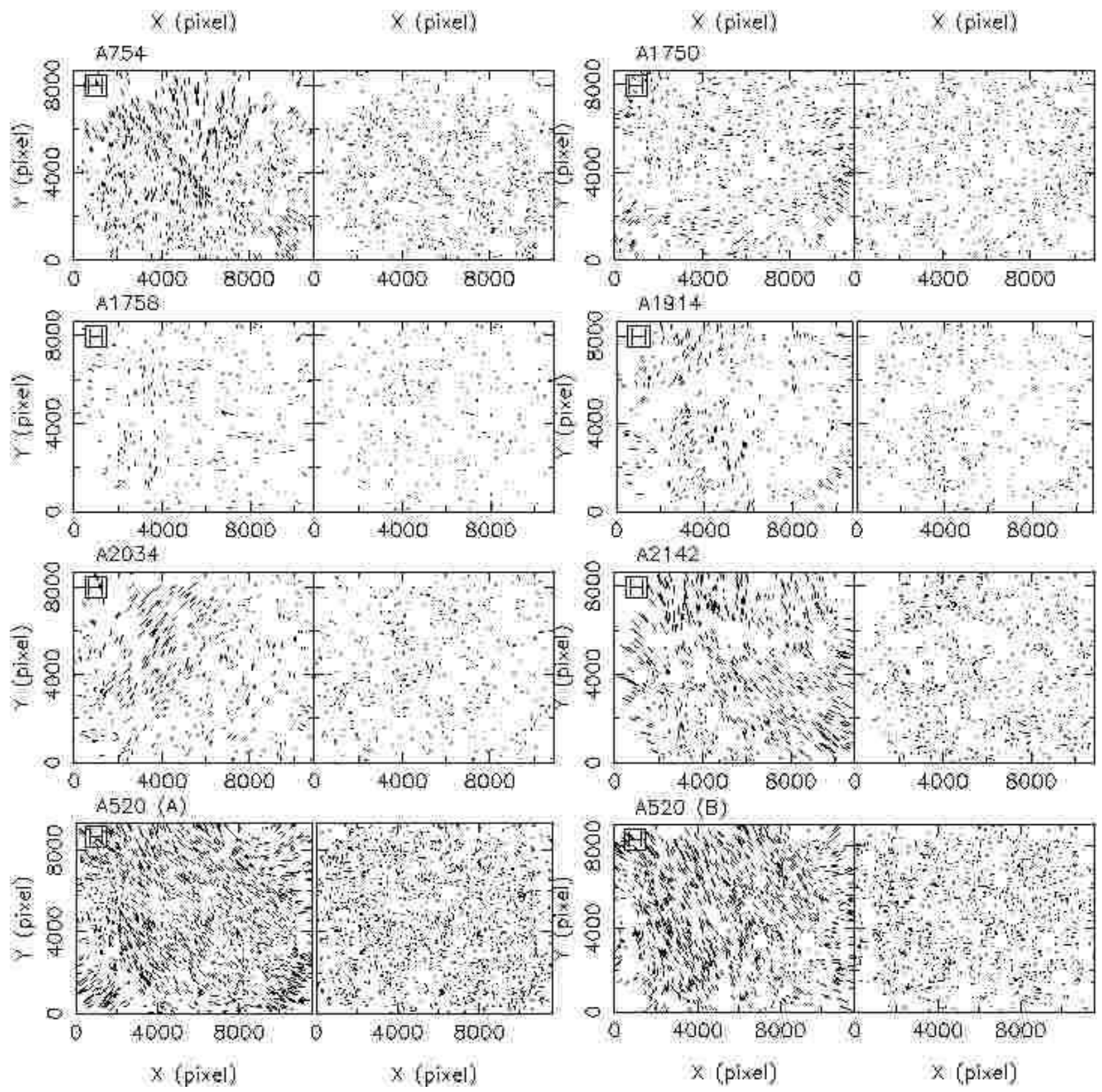

Fig. 4. The distortion field of stellar ellipticities before and after the PSF anisotropy correction for individual cluster targets. For each cluster the left panel shows the raw ellipticity field of stellar objects, and the right panel shows the residual ellipticity field after the PSF anisotropy correction. The orientation of the sticks indicates the position angle of the major axis of stellar ellipticity, whereas the length is proportional to the modulus of stellar ellipticity. A stick with the length of $10 \%$ ellipticity is indicated in the top left of the left panel for each target. For A520 two different imaging data sets are shown: A520 (A) is based on a co-added mosaic of seven $i^{\prime}$ images $(7 \times 240 \mathrm{~s})$ taken both with and without guide probe. A520 (B) is based on a co-added mosaic of four $i^{\prime}$ images $(4 \times 240$ s $)$ taken without guide probe. 

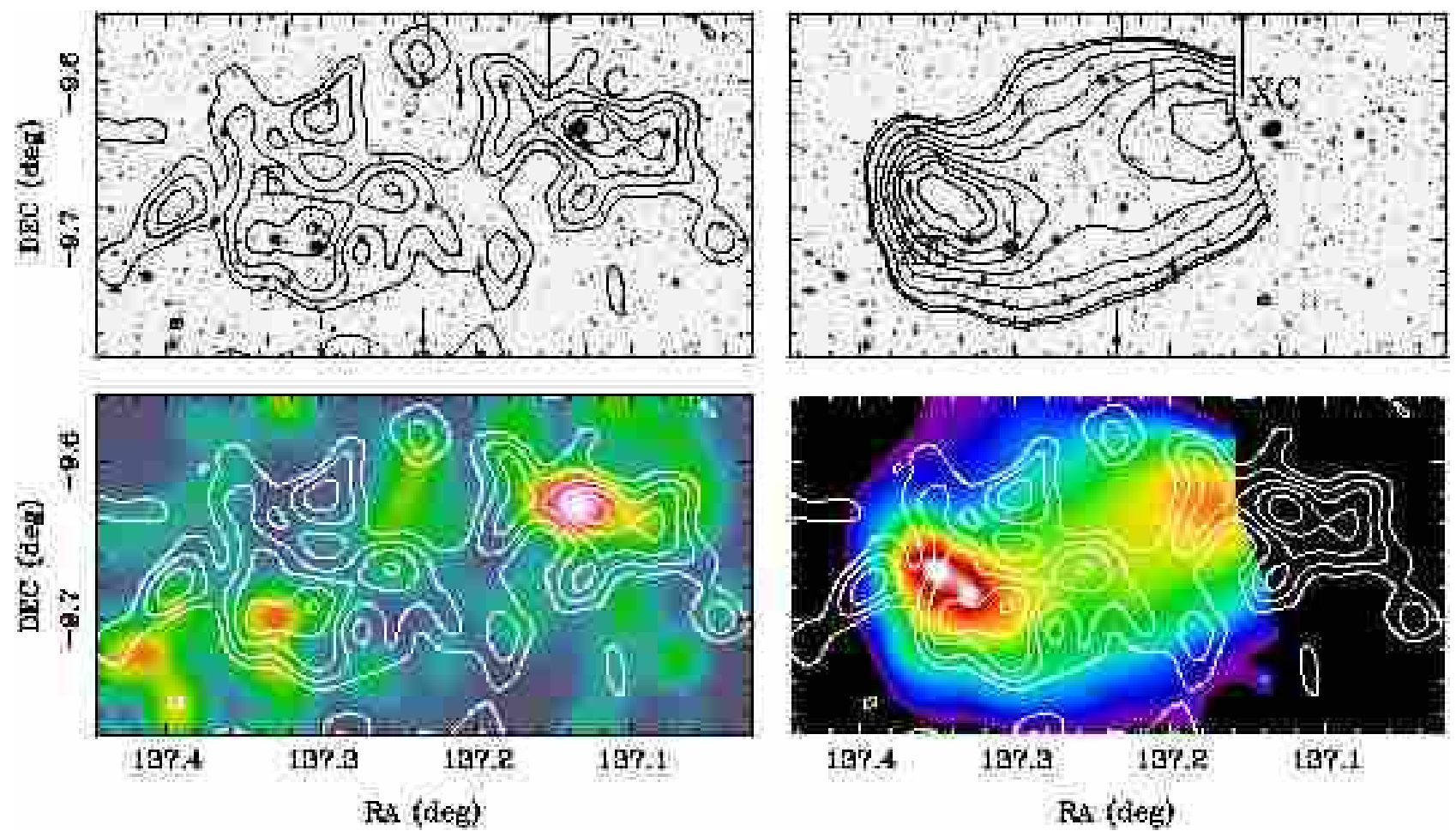

Fig. 5. A754 - Top-left: Subaru $R_{\mathrm{C}}$-band image of the central $\sim 25^{\prime} \times 13^{\prime}$ cluster region. Overlayed are contours of the lensing $\kappa$-field reconstructed from weak shear data. The contours are spaced in units of $1 \sigma$ reconstruction error (see Table 5). The Gaussian FWHM used for the mass reconstruction is 1!67. Top-right: adaptively-smoothed Chandra X-ray contours $(0.7-7.0 \mathrm{keV})$ overlaid on the same $R_{\mathrm{C}}$-band image. Bottom-left: Cluster luminosity density distribution in $R_{\mathrm{C}}$-band smoothed to the same angular resolution of the mass map. Overlayed are the same mass contours as in the top-left panel. Bottom-right: The same mass contours overlayed on the adaptively-smoothed Chandra X-ray image $(0.7-7.0 \mathrm{keV})$. 

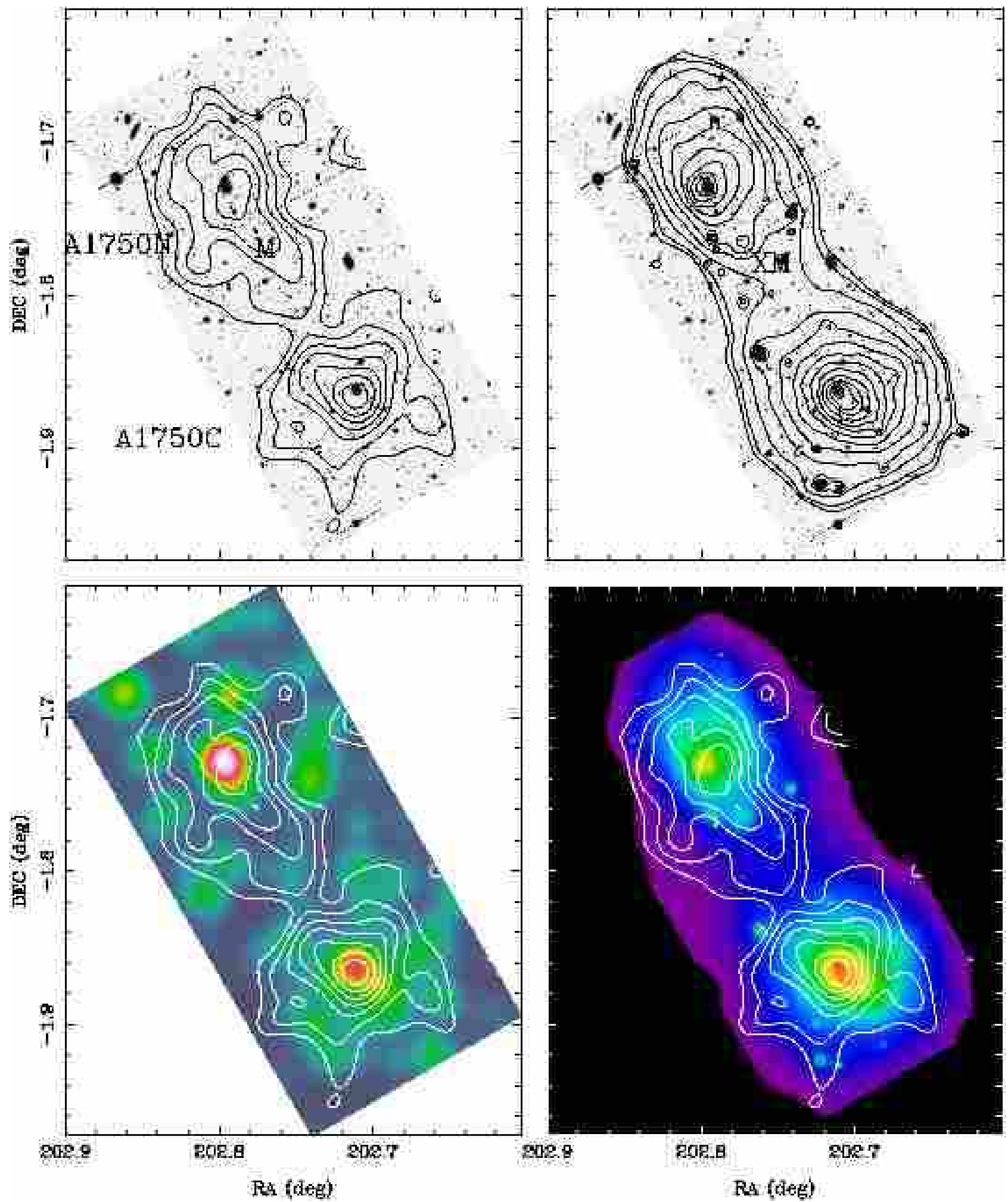

Fig. 6. A1750 - Top-left: Subaru $R_{\mathrm{C}}$-band image of the central $\sim 21^{\prime}, \times 18^{\prime}$ cluster region. Overlayed are contours of the lensing $\kappa$-field reconstructed from weak shear data. The contours are spaced in units of $1 \sigma$ reconstruction error (see Table 5 ). The Gaussian FWHM used for the mass reconstruction is 1!25. Top-right: adaptively-smoothed XMM-Newton X-ray contours $(0.5-7.0 \mathrm{keV})$ overlaid on the same $R_{\mathrm{C}}$-band image. Bottom-left: Cluster luminosity density distribution in $R_{\mathrm{C}}$-band smoothed to the same angular resolution of the mass map. Overlayed are the same mass contours as in the top-left panel. Bottom-right: The same mass contours overlayed on the adaptively-smoothed XMM-Newton X-ray image $(0.5-7.0 \mathrm{keV})$. 

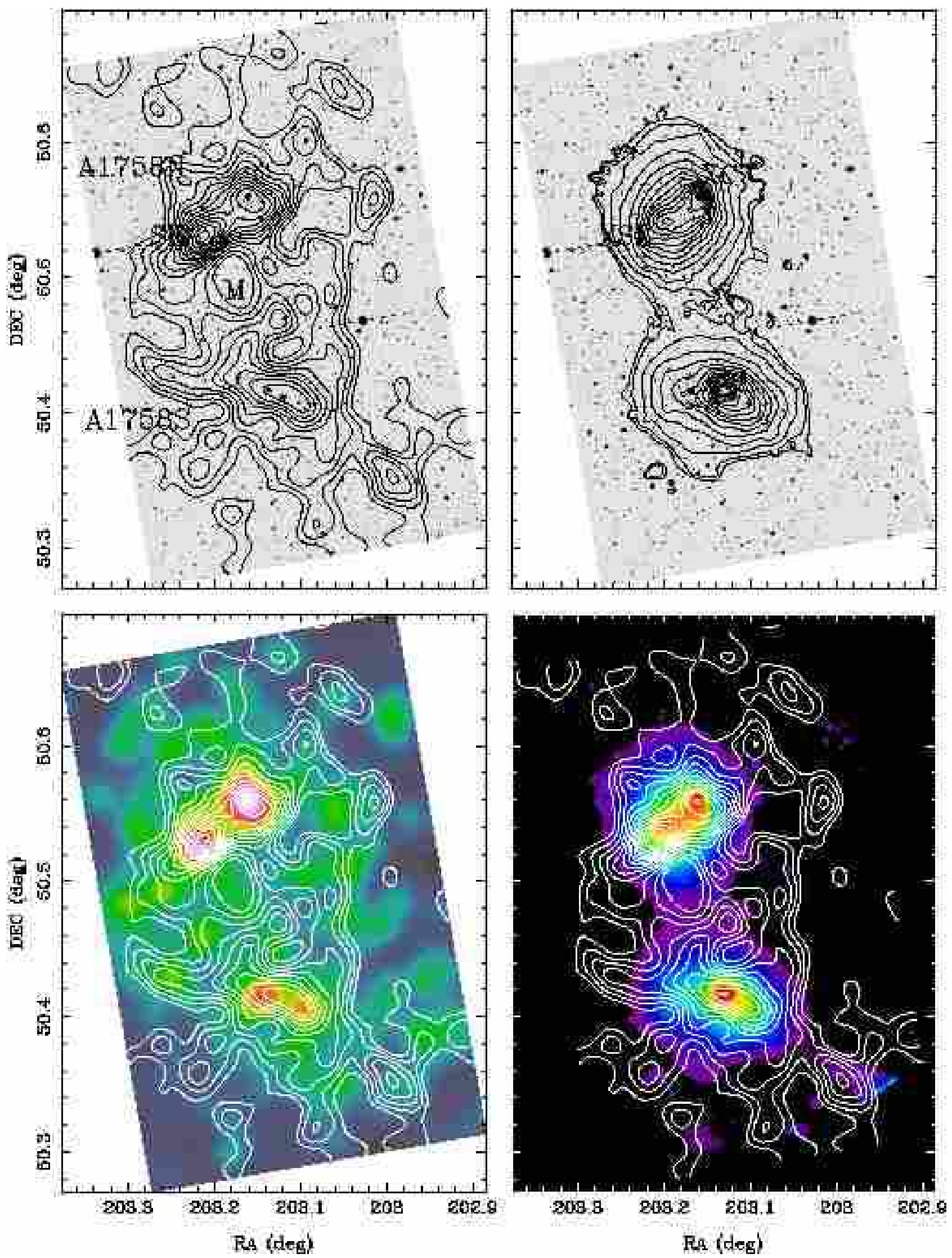

Fig. 7. A1758 - Top-left: Subaru $R_{\mathrm{C}}$-band image of the central $\sim 26^{\prime}, \times 19^{\prime}$ cluster region. Overlayed are contours of the lensing $\kappa$-field reconstructed from weak shear data. The contours are spaced in units of $1 \sigma$ reconstruction error (see Table 5 ). The Gaussian FWHM used for the mass reconstruction is 1 ! 25 . Top-right: adaptively-smoothed XMM-Newton X-ray contours $(0.5-7.0$ keV) overlaid on the same $R_{\mathrm{C}}$-band image. Bottom-left: Cluster luminosity density distribution in $R_{\mathrm{C}}$-band smoothed to the same angular resolution of the mass map. Overlayed are the same mass contours as in the top-left panel. Bottom-right: The same mass contours overlayed on the adaptively-smoothed XMM-Newton X-ray image $(0.5-7.0 \mathrm{keV})$. 

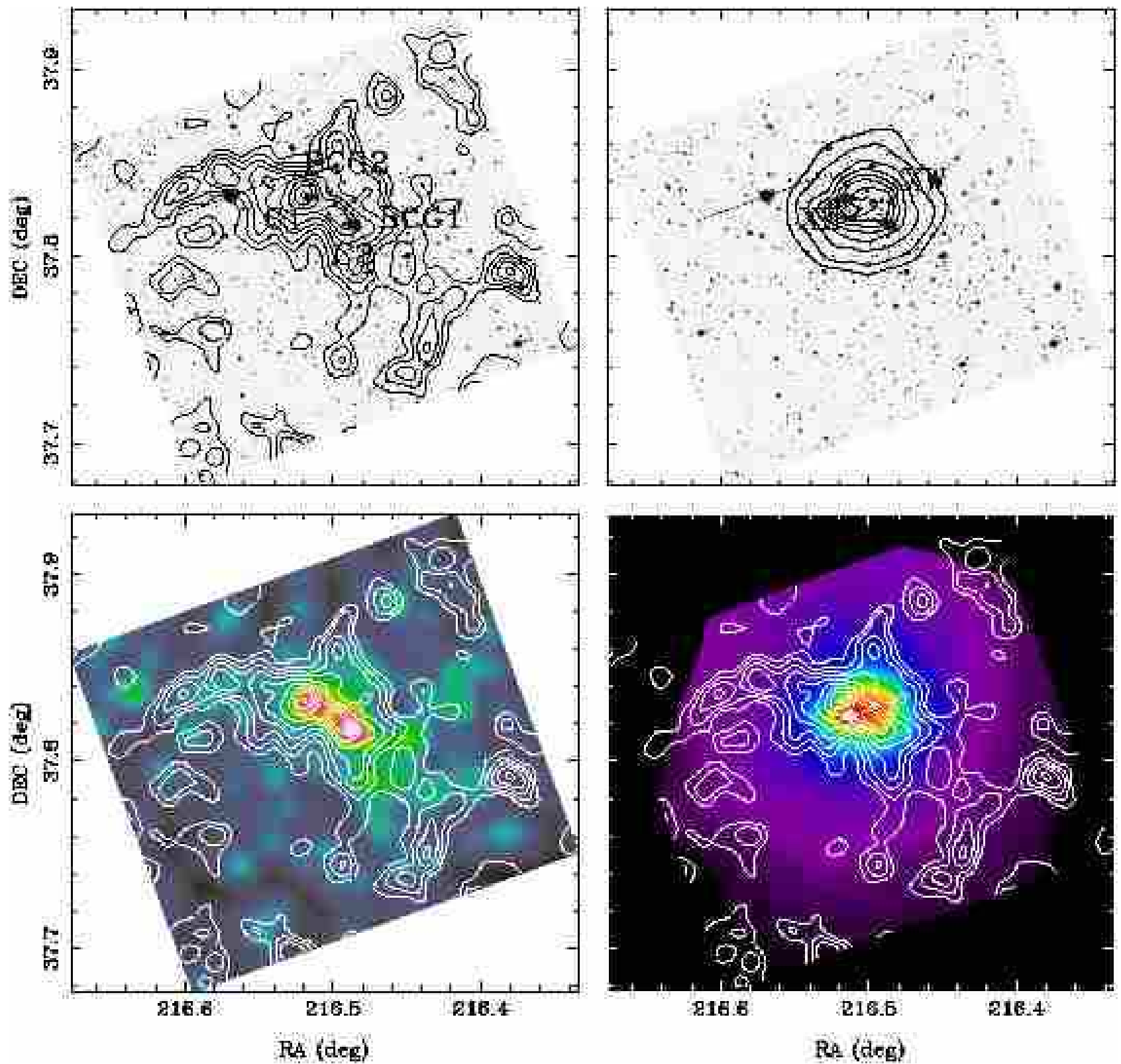

Fig. 8. A1914 - Top-left: Subaru $R_{\mathrm{C}}$-band image of the central $\sim 16^{\prime}, \times 15 ! 5$ cluster region. Overlayed are contours of the lensing $\kappa$-field reconstructed from weak shear data. The contours are spaced in units of $1 \sigma$ reconstruction error (see Table 5 ). The Gaussian FWHM used for the mass reconstruction is 0!75. Top-right: adaptively-smoothed Chandra X-ray contours $(0.7-7.0 \mathrm{keV})$ overlaid on the same $R_{\mathrm{C}}$-band image. Bottom-left: Cluster luminosity density distribution in $R_{\mathrm{C}}$-band smoothed to the same angular resolution of the mass map. Overlayed are the same mass contours as in the top-left panel. Bottom-right: The same mass contours overlayed on the adaptively-smoothed Chandra X-ray image $(0.7-7.0 \mathrm{keV})$. 

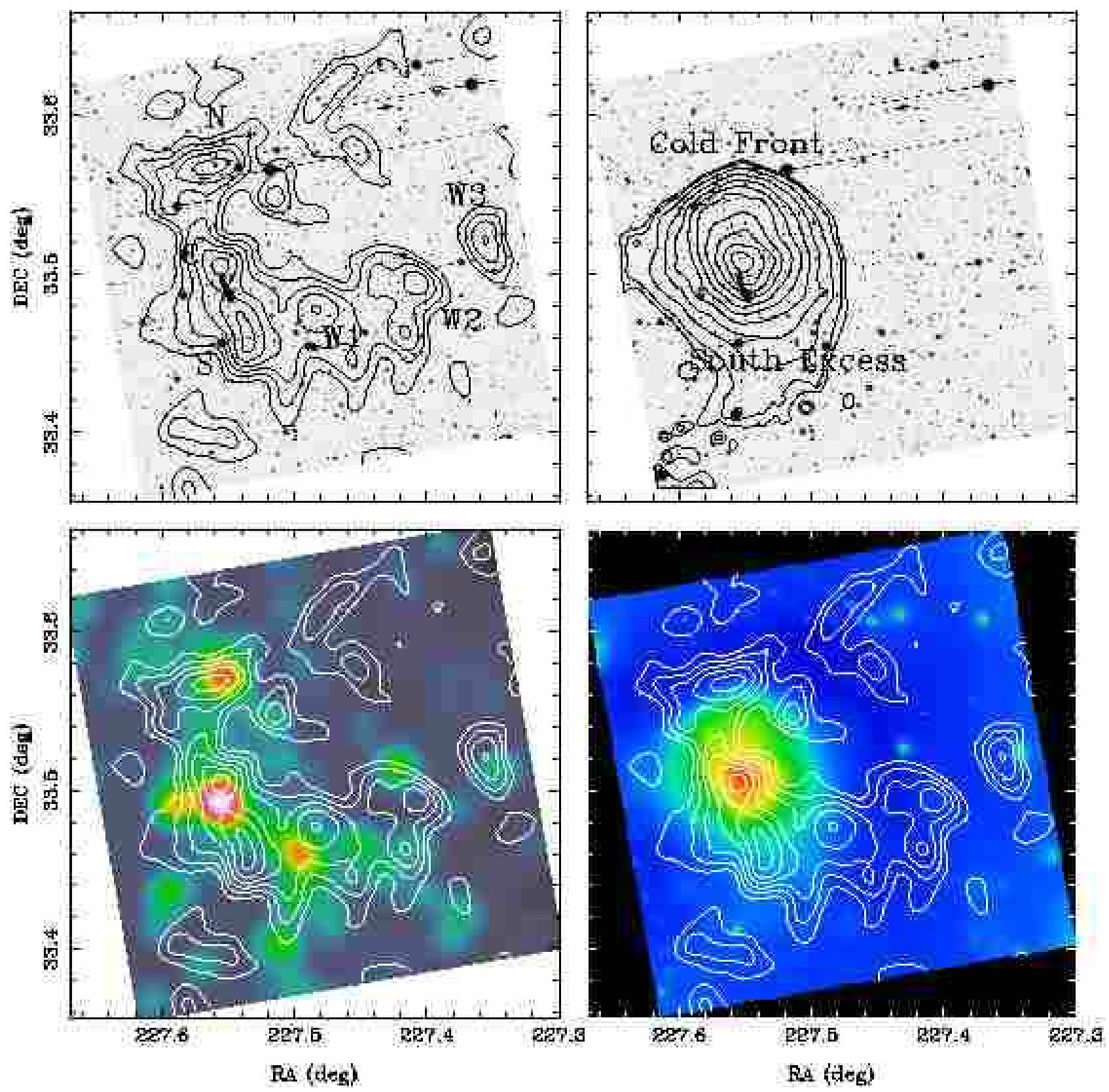

Fig. 9. A2034 - Top-left: Subaru $R_{\mathrm{C}}$-band image of the central $\sim 18.5, \times 18.5$ cluster region. Overlayed are contours of the lensing $\kappa$-field reconstructed from weak shear data. The contours are spaced in units of $1 \sigma$ reconstruction error (see Table 5 ). The Gaussian FWHM used for the mass reconstruction is 1 ! 17 . Top-right: adaptively-smoothed XMM-Newton X-ray contours $(0.5-7.0 \mathrm{keV})$ overlaid on the same $R_{\mathrm{c}}$-band image. Bottom-left: Cluster luminosity density distribution in $R_{\mathrm{C}}$-band smoothed to the same angular resolution of the mass map. Overlayed are the same mass contours as in the top-left panel. Bottom-right: The same mass contours overlayed on the adaptively-smoothed XMM-Newton X-ray image $(0.5-7.0 \mathrm{keV})$. 

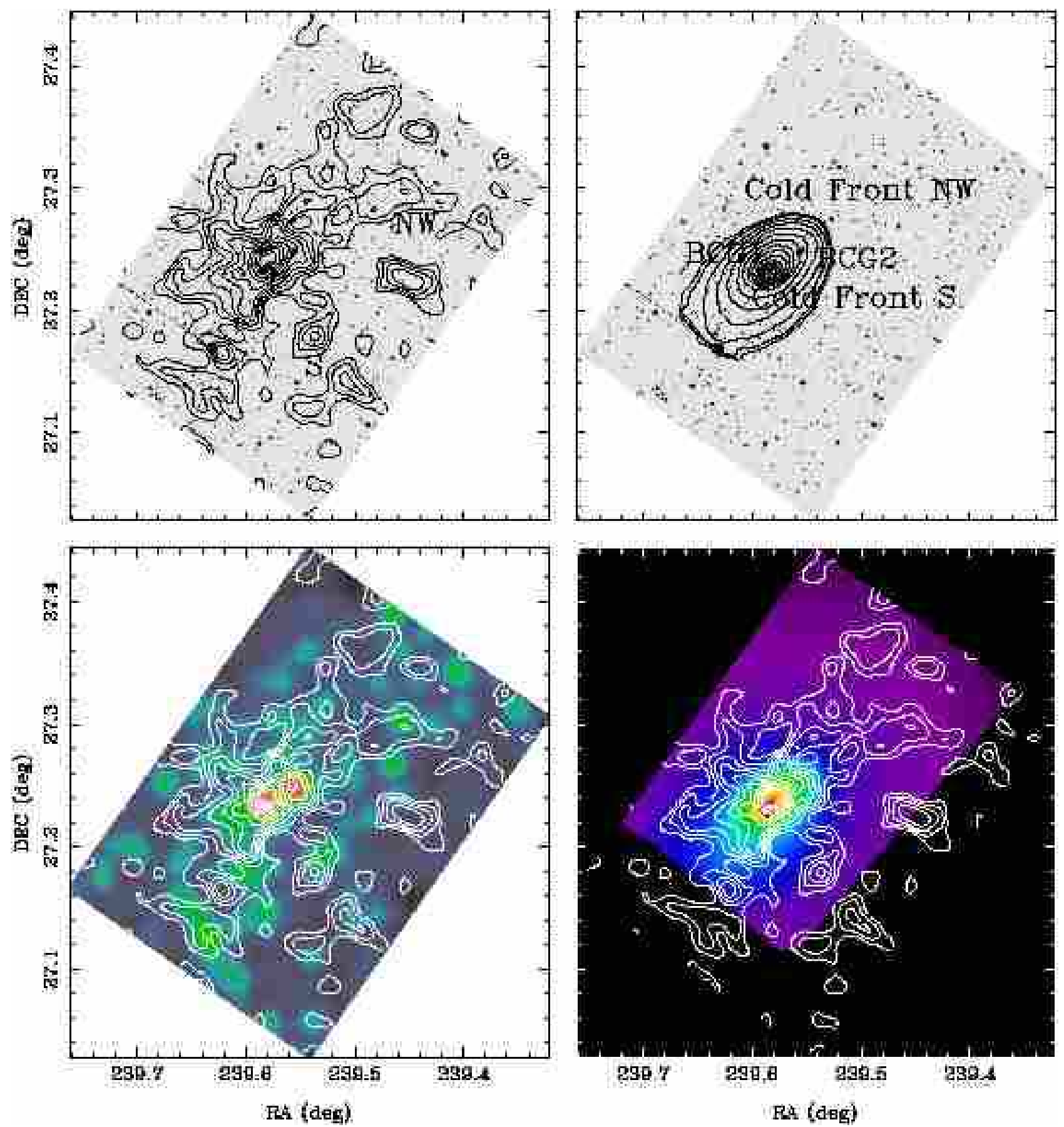

Fig. 10. A2142 - Top-left: Subaru $R_{\mathrm{C}}$-band image of the central $\sim 25^{\prime}, \times 23 \prime .5$ cluster region. Overlayed are contours of the lensing $\kappa$-field reconstructed from weak shear data. The contours are spaced in units of $1 \sigma$ reconstruction error (see Table 5 ). The Gaussian FWHM used for the mass reconstruction is 1'.00. Top-right: adaptively-smoothed Chandra X-ray contours $(0.7-7.0 \mathrm{keV})$ overlaid on the same $R_{\mathrm{C}}$-band image. Bottom-left: Cluster luminosity density distribution in $R_{\mathrm{C}}$-band smoothed to the same angular resolution of the mass map. Overlayed are the same mass contours as in the top-left panel. Bottom-right: The same mass contours overlayed on the adaptively-smoothed Chandra X-ray image $(0.7-7.0 \mathrm{keV})$. 

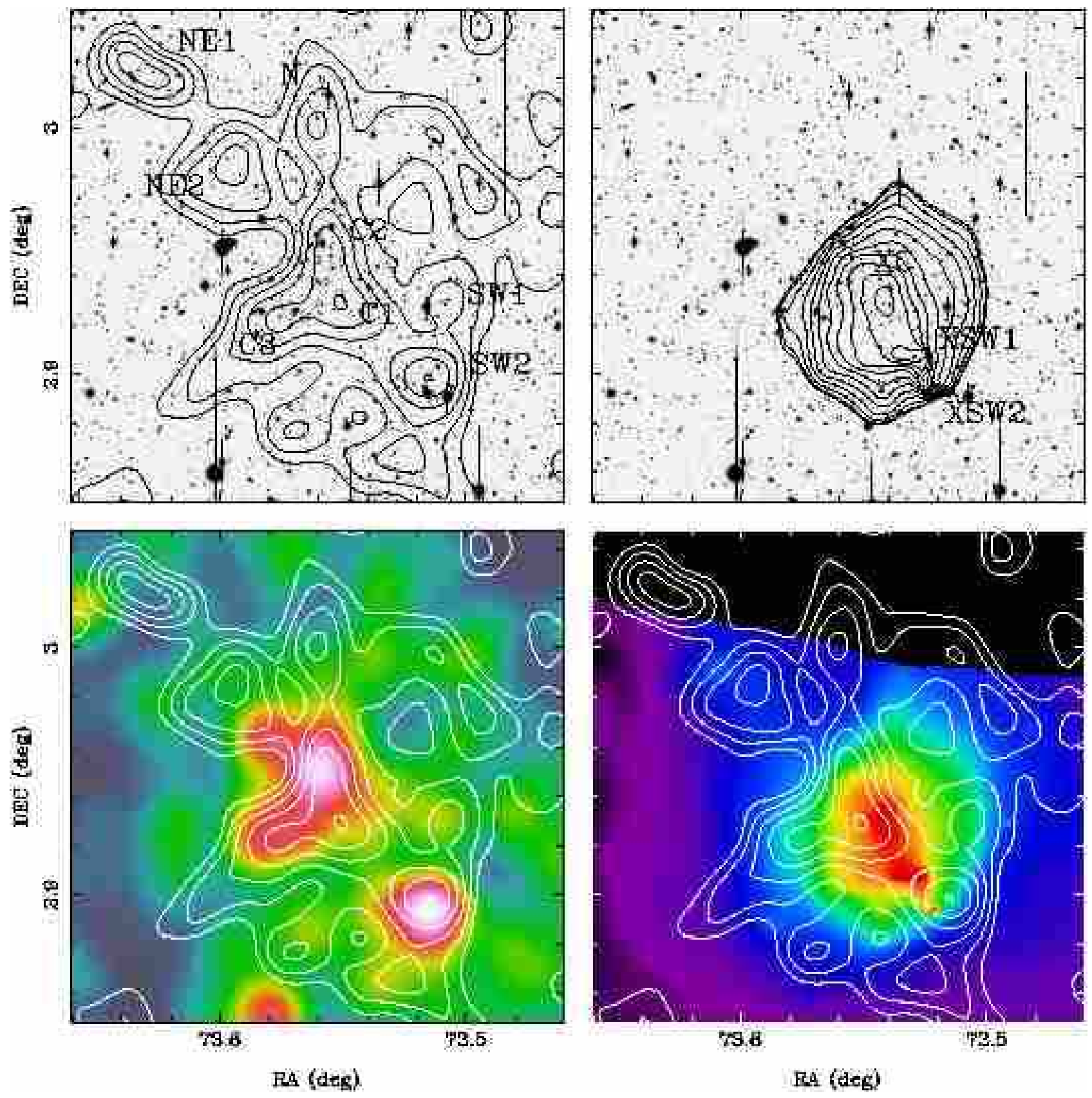

Fig. 11. A520 - Top-left: Subaru $i^{\prime}$-band image of the central $\sim 12^{\prime}, \times 12^{\prime}$ cluster region. Overlayed are contours of the lensing $\kappa$-field reconstructed from weak shear data. The contours are spaced in units of $1 \sigma$ reconstruction error (see Table 5 ). The Gaussian FWHM used for the mass reconstruction is 1 ! 25 . Top-right: adaptively-smoothed Chandra X-ray contours $(0.7-7.0 \mathrm{keV})$ overlaid on the same $i^{\prime}$-band image. Bottom-left: Cluster luminosity density distribution in $i^{\prime}$-band smoothed to the same angular resolution of the mass map. Overlayed are the same mass contours as in the top-left panel. Bottom-right: The same mass contours overlayed on the adaptively-smoothed Chandra X-ray image $(0.7-7.0 \mathrm{keV})$. 

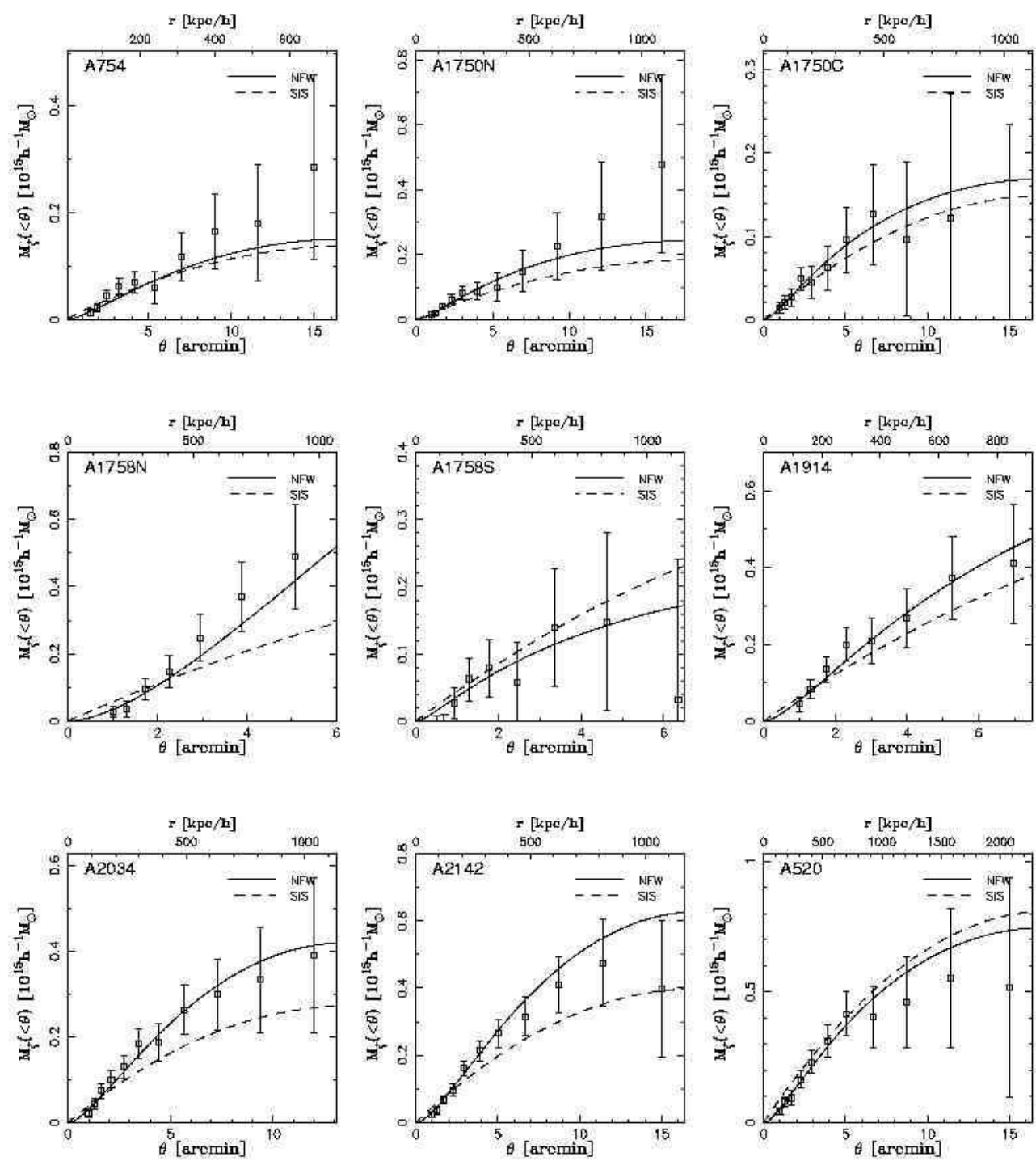

Fig. 12. Radial profiles of the projected mass $M_{\zeta}(\theta)$ for a sample of seven merging clusters of galaxies as measured by the weak lensing $\zeta$-statistic. The error bars are correlated. Also plotted are the best-fitting NFW and SIS profiles. 


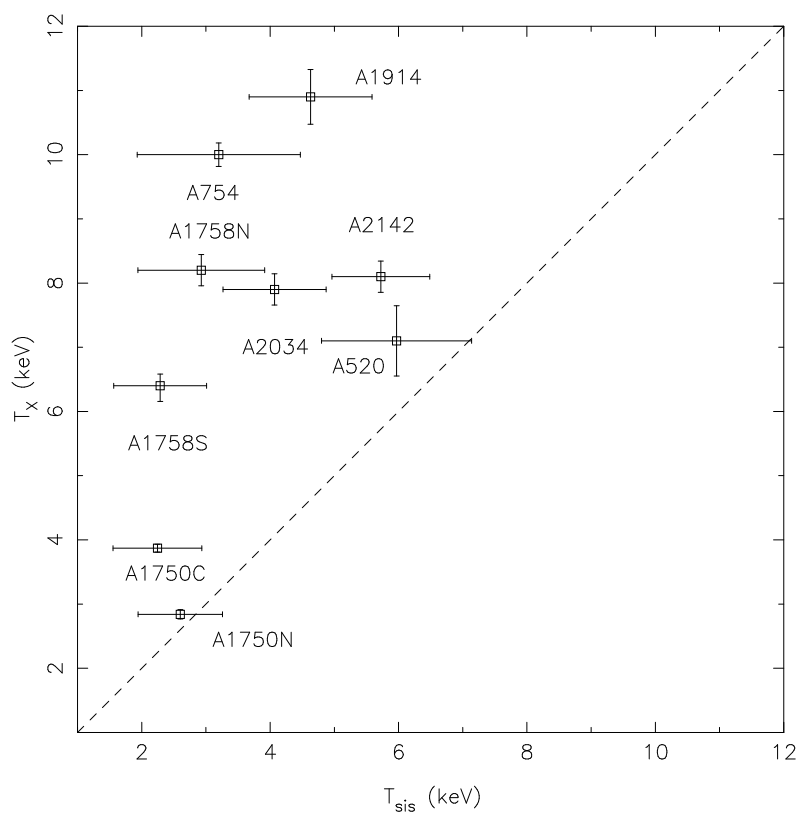

Fig. 13. Comparison between the best-fitting SIS temperature $T_{\mathrm{SIS}}$ from the $\zeta_{c}$-statistic measurement vs. the averaged X-ray temperature $T_{X}$ for a sample of nearby merging clusters. The dashed line indicates $T_{\mathrm{SIS}}=T_{X}$. For all clusters except A1750N, the observed X-ray temperature is significantly higher than the expected virial temperature of the cluster mass.

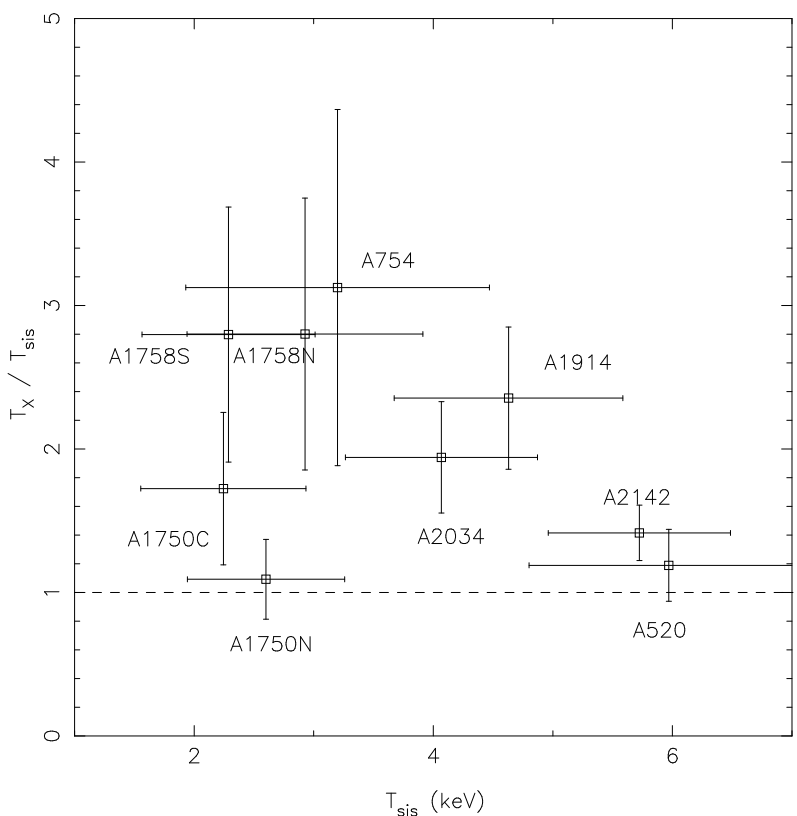

Fig. 14. The temperature ratio $T_{X} / T_{\mathrm{SIS}}$ v.s. the best-fitting SIS temperature $T_{\mathrm{SIS}}$. The dashed line indicates $T_{\mathrm{SIS}}=T_{X}$. 


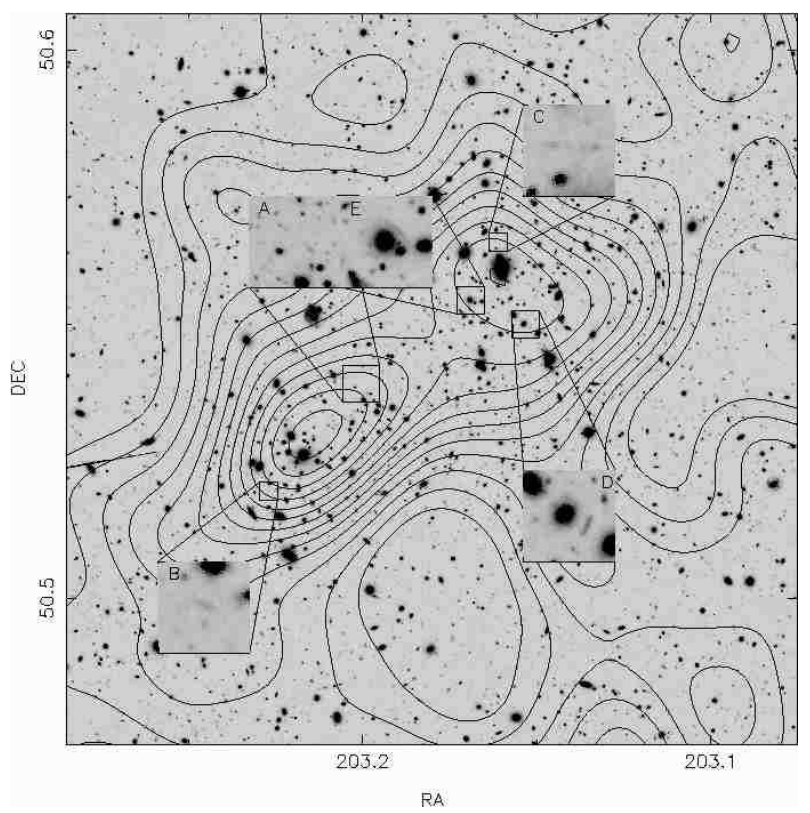

Fig. 15. Subaru $R_{\mathrm{C}}$-band image of the central 8! $\times 8$ ! cluster region of A1758N. Overlayed are contours of the reconstructed projected mass distribution of the cluster. The insets zoom in on tangential arc candidates identified based on visual inspection. The panels A $(0,4 \times 0 ! 4)$ and B $(0,2 \times 0 ! 2)$ are zoom in of blue tangential arcs around the SE mass clump. The panel C $(0 ! 2 \times 0 ! 2)$ is a zoom in view of blue tangential arcs around the mass clump $\mathrm{C}$. The panels $\mathrm{D}(0,3 \times 0,3)$ and $\mathrm{E}(0,3 \times 0,3)$ are zoom in of tangential arcs associated with cluster galaxy concentrations.

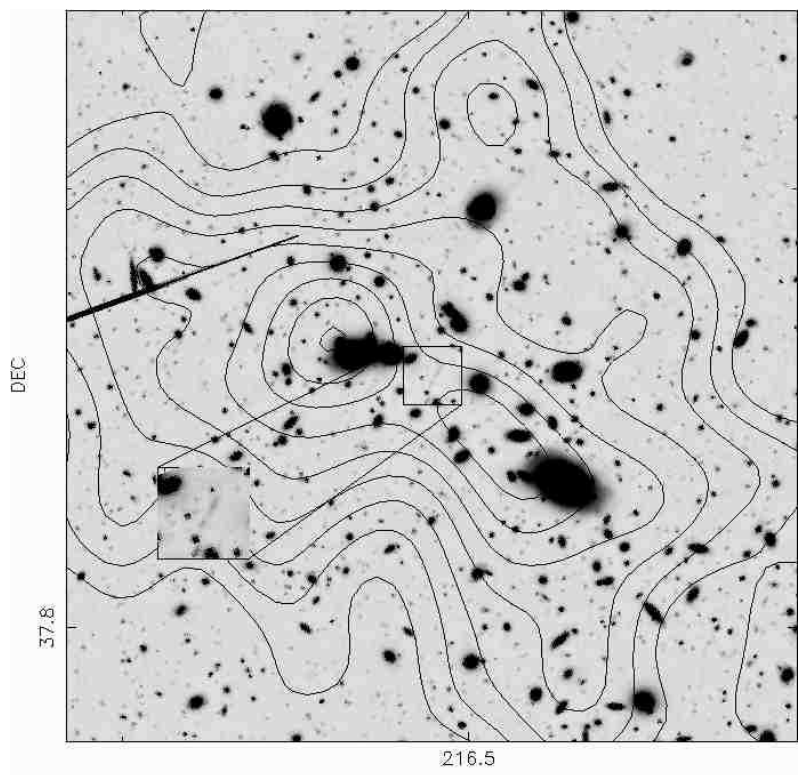

RA

Fig. 16. Subaru $R_{\mathrm{C}}$-band image of the central 5! $\times 5$ ! cluster region of A1914. Overlayed are contours of the reconstructed projected mass distribution of the cluster. The inset panel $(0,4 \times 0 ! 4)$ shows a zoom in view of two blue tangential arcs around BCG2. 


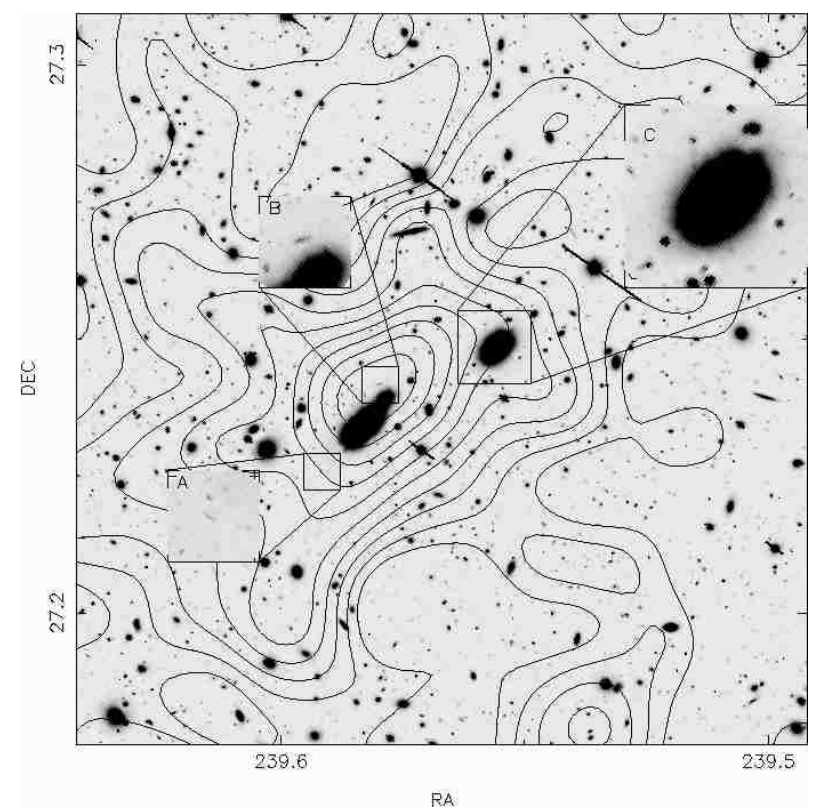

Fig. 17. Subaru $R_{\mathrm{C}}$-band image of the central 8! $\times 8$ ! cluster region of A2142. Overlayed are contours of the reconstructed projected mass distribution of the cluster. The insets zoom in on tangential arc candidates identified based on visual inspection. The panel A shows a zoom in view $(0 ! 4 \times 0 ! 4)$ of a tangential arc. The panel B shows a zoom in view $(0.4 \times 0.4)$ of a tangential arc. The panel $\mathrm{C}$ sows a zoom in view $(0 ! 8 \times 0 ! 8)$ around $\mathrm{BCG} 2$, where no arc-like image is found by visual inspection. 

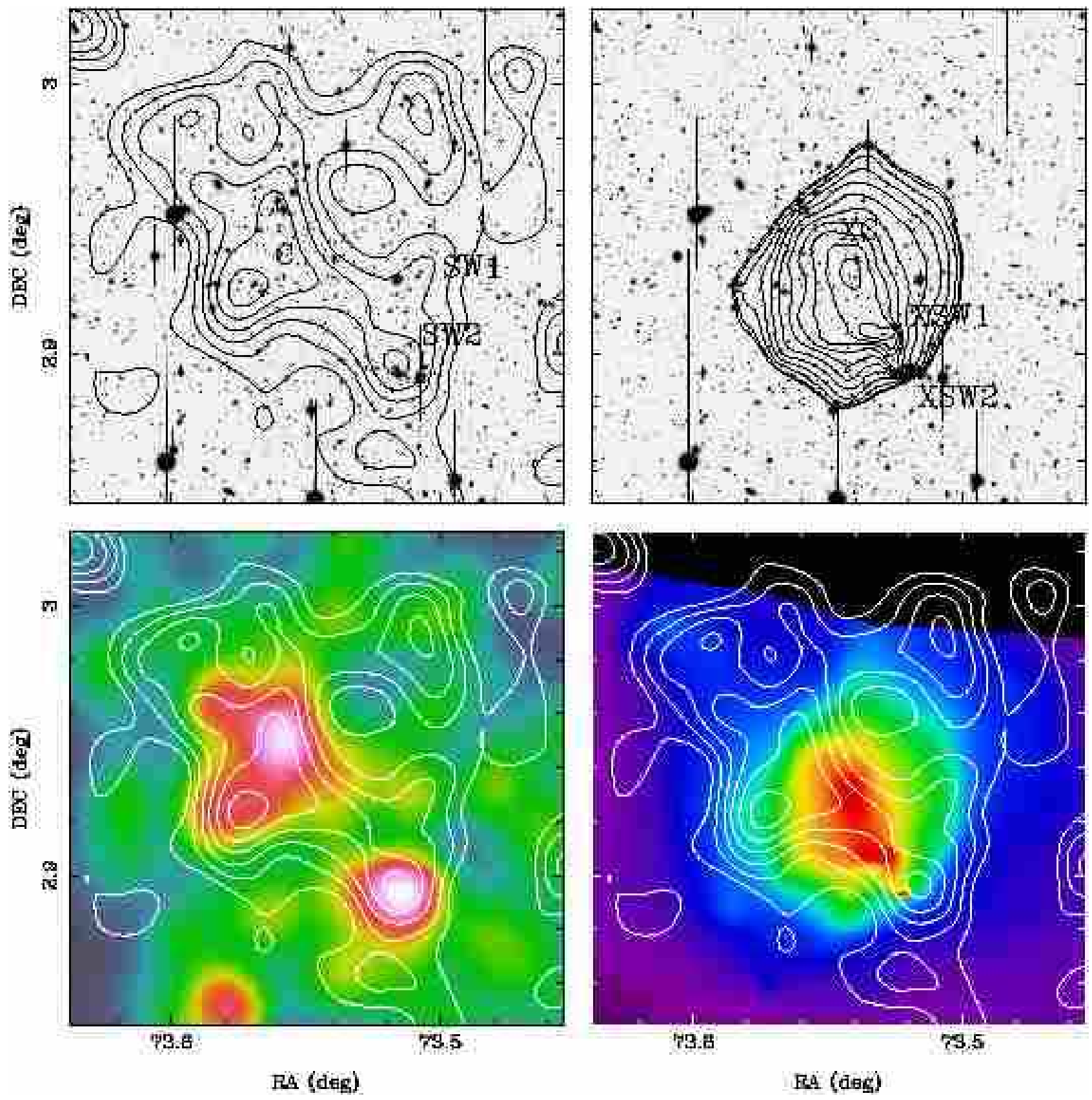

Fig. 18. A520 based on $4 \times 240$ s $i^{\prime}$-band images taken without guide star - Top-left: Subaru $i^{\prime}$-band image of the central $\sim 11^{\prime}, \times 11^{\prime}$ cluster region. Overlayed are contours of the lensing $\kappa$-field reconstructed from weak shear data. The contours are spaced in units of $1 \sigma$ reconstruction error (see Table 5). The Gaussian FWHM used for the mass reconstruction is 1!25. Top-right: adaptively-smoothed Chandra X-ray contours $(0.7-7.0 \mathrm{keV})$ overlaid on the same $i^{\prime}$-band image. Bottom-left: Cluster luminosity density distribution in $i^{\prime}$-band smoothed to the same angular resolution of the mass map. Overlayed are the same mass contours as in the top-left panel. Bottom-right: The same mass contours overlayed on the adaptively-smoothed Chandra X-ray image $(0.7-7.0 \mathrm{keV})$. 


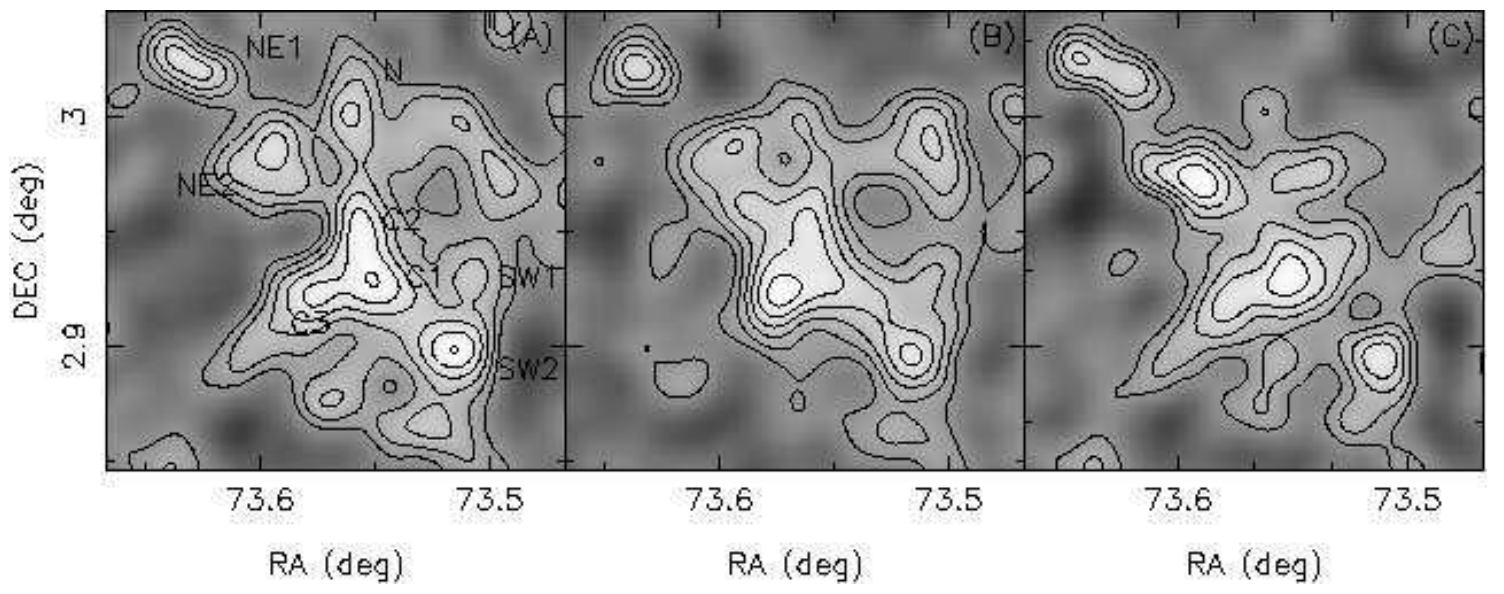

Fig. 19. Comparisons of A520 weak lensing analyses of different data sets. Left (A) : The lensing $\kappa$-field of $(12$ ! $\times 12$ '), reconstructed from seven $i^{\prime}$ images with and without AG (acquisition and guide) probe $(7 \times 240$ s exposure), which was also used by Mahdavi et al. (2007). The Gaussian FWHM used for the mass reconstruction is 1'25. The contours are spaced in units of $1 \sigma$ reconstruction error. Middle (B) : The same figure as left panel, reconstructed from four $i^{\prime}$ images taken without guide probe $(4 \times 240 \mathrm{~s}$ exposure). Right (C) : The same figure as left panel, reconstructed from three $i^{\prime}$ images taken with guide probe $(3 \times 240$ s exposure $)$. 

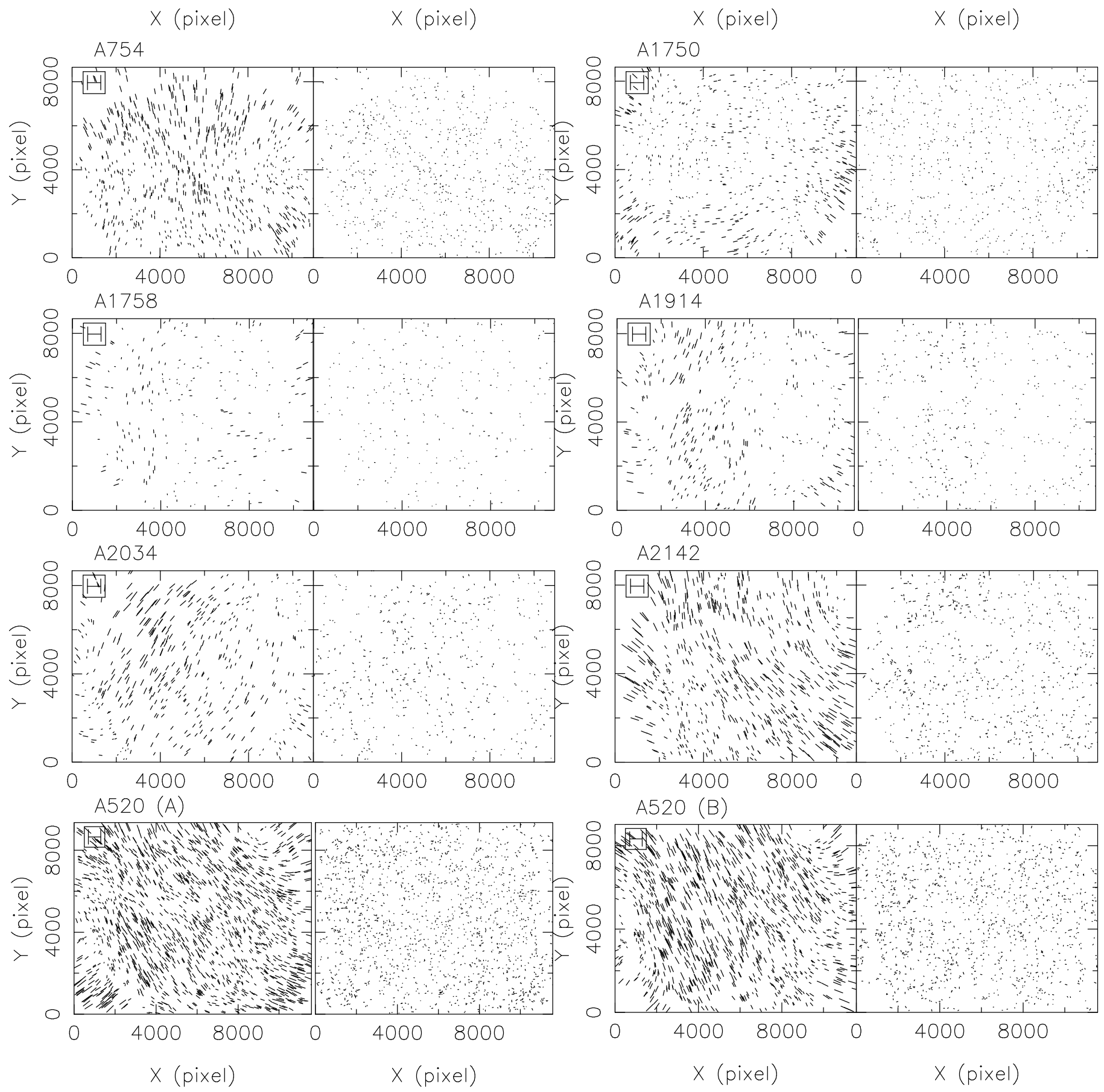